\title{
CATÁLOGO DE LOS REPTILES PROCEDENTES DE LA COMUNIDAD DE MADRID (ESPAÑA) QUE SE CONSERVAN EN EL MUSEO NACIONAL DE CIENCIAS NATURALES
}

\author{
J. E. González Fernández*
}

\begin{abstract}
RESUMEN
En el presente trabajo, se proporciona un catálogo actualizado de los reptiles de la Comunidad de Madrid (España) elaborado gracias a la información disponible en los ficheros y libros de colección del MNCN. Se han confirmado los datos que figuraban en los antiguos libros de registro, catálogo y fichas de colección referentes a los reptiles procedentes de Madrid existentes en la colección de anfibios y reptiles del MNCN, comprobándose que ejemplares se conservan en la actualidad. También se ha actualizado y comprobado que la información disponible en las bases de datos del MNCN era correcta y acorde con los datos existentes en los ejemplares. Los errores detectados han sido corregidos. Como consecuencia de ello se ha corroborado fehacientemente la existencia, a fecha 30 de noviembre de 2005, de 2237 ejemplares de reptiles de dicha procedencia, que se han incluido en 1039 registros informáticos. Entre los ejemplares depositados en la colección, destacan algunos colectados por Eduardo Boscá y Casanoves -padre de la Herpetología Española- y por Francisco de P. Martínez y Sáez -ex miembro de la Comisión Científica al Pacífico- (1862-1865), que se conservan en el MNCN desde hace 140 años. Junto a la documentación que les acompaña, todos estos ejemplares se guardan como parte integrante del Patrimonio Nacional común, estando a disposición de las futuras generaciones de investigadores, como testigos de la biodiversidad pasada y actual. También se amplia, para algunas especies, su área de distribución en la Comunidad de Madrid.
\end{abstract}

Palabras clave: Herpetología, Catálogo museológico, Colecciones científicas, Reptiles, Comunidad de Madrid (España), Mapas de Distribución de Reptiles.

\section{ABSTRACT \\ Catalogue of the Reptiles from Comunidad de Madrid (Spain) preserved in the Museo Nacional de Ciencias Naturales}

A complete catalogue of reptiles found in Comunidad de Madrid (Spain) is provided in this paper. The catalogue is taxonomically indexed and listed in alphabetical order including all basic data. It includes all information available in card files and old collection books of the MNCN. We have confirmed which reptiles in the current collection appear as being collected in Madrid, in old records, catalogue books and collection cards in the collection of amphibians and reptiles of the MNCN. The information of these old databases has been verified and mistakes corrected. As a consequence of this revision, the existence of 2237 specimens of reptiles originating in the above-indicated area and

* Museo Nacional de Ciencias Naturales, CSIC; José Gutiérrez Abascal 2; E-28006; Madrid; jegonzalez@mncn.csic.es 
included in 1039 computer records, has been confirmed as of 30 November 2005. Among the oldest specimens included in the collection are some collected by the founding father of Spanish herpetology (Eduardo Boscá y Casanoves) or by a member of the Pacific Scientific Commission (Francisco de P. Martínez y Sáez) and deposited in the MNCN more than 140 years ago. These specimens and their data are preserved at the MNCN as part of our common National Heritage, for future generations of researchers as witnesses of the past and present biodiversity. Distribution areas for several species have been extended.

Keywords: Herpetology, Museological catalogue, Scientific collections, Reptiles, Comunidad de Madrid (Spain), Reptiles Distribution Maps.

\section{Introducción}

La historia de la colección de anfibios y reptiles del Museo Nacional de Ciencias Naturales comienza con la fundación del Real Gabinete de Historia Natural (RGHN) por Carlos III en 1771, al adquirir la Corona la colección y biblioteca de Pedro Franco Dávila (quien posteriormente sería nombrado primer director del entonces denominado RGHN) y enriquecerlos con otros materiales ya en poder de la Corona (González Fernández, 1997). Con posterioridad, el RGHN tras diversas modificaciones pasó a llamarse Museo Nacional de Ciencias Naturales. Parte de sus fondos se emplearon para dotar inicialmente al Real Jardín Botánico, al Museo Antropológico y al Museo Arqueológico Nacional (Borbón y Grecia, 2001).

Aunque el catálogo manuscrito confeccionado por el catedrático de Anatomía Comparada y Director del MNCN, Mariano de la Paz Graells (Graells, 1846), recoge algunos reptiles, la parquedad de los datos que los acompañan es tal que es imposible asignar localidades o ejemplares concretos de la colección actual a los mismos, pues tan sólo habla de la existencia de ejemplares de los géneros Lacerta Linnaeus, 1758; Psammodromus Fitzinger, 1826; Acanthodactylus Wiegmann, 1834; Chalcides Laurenti, 1768 Coluber Oppel, 1811 y Natrix Laurenti, 1768.

Durante la década de los setenta del pasado siglo XX, hubo dos intentos de acometer el inventario y ordenación de la coleción de anfibios y reptiles (González-Fernández, 2002), intentos que aunque fallidos, constituyeron un esbozo de la tarea que posteriormente se realizó. Hubo que esperar hasta 1985 cuando bajo la dirección de Emiliano Aguirre, se afrontó el objetivo de recuperar globalmente las colecciones del MNCN. Durante los 20 años transcurridos desde entonces, se han sentado las bases para la ordenación (Fernandez et al., 1992), conservación (Barreiro et al., 1994), inven- tario (Fernández et al., 1992), informatización y catalogación (González-Fernández, 2002), así como de la recuperación de la información. Los resultados de este esfuerzo comienzan a plasmarse en catálogos generales que nos permiten conocer el material realmente existente en las colecciones del MNCN, así como del potencial de la información de la que son portadores (Martínez-Solano \& González-Fernández, 2003) y de lo cual el presente trabajo es una muestra.

En este trabajo proporcionamos el catálogo de los reptiles procedentes de la Comunidad de Madrid que forman parte de las colecciones del MNCN. Madrid se sitúa la zona centro peninsular en la parte más Noroeste de la meseta inferior, ocupa una superficie de $7995 \mathrm{~km}^{2}$, lo que representa una pequeña parte, tan solo el $1^{\prime} 6 \%$, del territorio español (García Ballesteros et al., 1991). Bajo el punto de vista biogeográfico, la Comunidad de Madrid pertenece al reino Holártico, región Mediterránea (occidental), y formando parte de dos provincias significativamente diferente: $\mathrm{La}$ Carpetano-Ibero-Leonesa (sector Guadarrámico, subsector Guadarramense y Matritense) y la Castellano-Maestrazgo-Manchega (sector Manchego, subsector Sagrense) (Rivas-Martínez, 1987). Bioclimáticamente el piso dominante es el Mesomediterráneo, aunque con sectores de tipo Supramediterráneo y puntos del Oromediterráneo y Criomediterráneo en las cumbres más elevadas (Rivas-Martínez, 1987). Además presenta una variación altitudinal desde los $480 \mathrm{~m}$ de la zona suroeste de la provincia, hasta los $2430 \mathrm{~m}$. del pico Peñalara, como "techo" de la Comunidad de Madrid. Situada al sur del sistema central (que separa la meseta superior de la inferior), Madrid, pertenece a la cuenca del Tajo y está cruzada por los ríos Tajuña, Henares, Jarama, Manzanares, Guadarrama y Alberche (Arija, 1972).

Entre los objetivos del trabajo quisiéramos destacar el deseo de hacer llegar la información exis- 
tente sobre los reptiles madrileños conservados en el MNCN (y los datos que les acompañan) no sólo a los herpetólogos u otros investigadores profesionales, sino que también por la faceta museológica y divulgativa que el MNCN realiza, nos gustaría que dicho conocimiento pudiera ser extensivo al gran público: aficionados, naturalistas, o amantes de la naturaleza. También nos gustaría que sirviera para que las personas físicas o jurídicas y las Instituciones públicas o privadas, legalmente poseedoras de ejemplares dignos de incorporarse a una colección científica, se animen a donar dichos especímenes al MNCN, la Institución pública española poseedora de las colecciones zoológicas ibéricas más importantes del mundo y de la colección zoológica más importante de España. En este contexto no queremos olvidar que junto a la investigación y la divulgación, uno de los tres objetivos definitorios y básicos de este Museo, es la conservación de las colecciones científicas.

Los reptiles recogidos en la base de datos del MNCN a fecha 31 de octubre de 2005, están incluidos en 1039 registros informáticos y suponen un total 2237 ejemplares, pertenecientes a las familias Amphisbaenidae, Bataguridae, Chamaeleontidae, Colubridae, Emydidae, Gekkonidae, Iguanidae, Lacertidae, Scincidae y Viperidae. En los casos de los dos únicos ejemplares de las familias Chamaeleontidae e Iguanidae, sin duda se trata de introducciones accidentales que simplemente recogemos aquí como curiosidad. En el primer caso, un ejemplar de Chamaeleo chamaeleon (Linnaeus, 1758), sin duda se trata de la captura de un ejemplar mantenido en cautividad, tras su fuga o liberación. El segundo caso, un iguánido, probablemente perteneciente al género Anolis Daudin, 1802, colectado en las cercanías del madrileño aeropuerto de Barajas que, con toda probabilidad, debe corresponder a un ejemplar escapado de un avión procedente de América.

\section{Material y métodos}

La información recogida en las etiquetas originales confeccionadas por los colectores, así como la existente en las fichas antiguas que había en la colección, fue el material de partida para la elaboración del presente trabajo. No obstante, en muchas ocasiones el tiempo transcurrido desde su colecta, así como los diferentes avatares históricos, políticos y de otra índole acaecidos en los más de 200 años de existencia del MNCN, produjeron que en demasiadas ocasiones la información aneja a los ejemplares se deteriorara, traspapelara, e incluso desapareciera, antes de iniciar las labores de recuperación en el año 1985. Por tanto la recuperación (a lo largo de todos estos años) de la información de la que eran portadores los ejemplares, ha sido en muchos casos una tarea ardua y complicada. Esta labor ha implicado muchas horas de investigación en el archivo y en antiguos libros de entradas y de registro del MNCN. A pesar de este esfuerzo desgraciadamente en algunos casos no se ha podido conseguir la correcta o completa datación.

Hasta el año 2000 los nuevos datos descubiertos en el archivo, libros de catálogo o fichas de colección antiguas, se incluían directamente en las bases de datos, por lo que una parte importante de la información añadida hasta esa fecha no es fácil de diferenciar de la que existía previamente al estudio y documentación moderno de las colecciones. Con posterioridad al año 2000, cuando algún dato (no taxonómico) no conocido anteriormente se añade a la base de datos, esta información se incluye entre corchetes: [....]. Esto tiene dos objetivos: (A) diferenciar claramente lo que se sabía de lo que no antes de la revisión, (B) poner "en cuarentena" los datos añadidos ante posibles revisiones posteriores o aparición de documentos históricos. Dentro de éstos documentos desaparecidos, cabría mencionar el catálogo manuscrito de los reptiles de la colección del Real Gabinete de Historia Natural, obra desaparecida y confeccionada en torno a 1822 por Eduardo Villanova, que fue uno de los méritos que le reconocieron para nombrarle Conservador, del entonces Real Museo de Historia Natural, en 1839 (Barreiro, 1992: 166-167).

\section{ACTUALIZACIÓN TAXONÓMICA}

Se ha actualizado la nomenclatura conforme a estos dos supuestos: (1) Cuando los ejemplares se encontraban identificados, la actuación ha consistido en comprobar que la denominación era correcta actualmente, corrigiéndose en aquellos casos en los que dicha determinación era errónea, mediante la actualización de sinonimias, o la eliminación de errores de clasificación. (2) Cuando carecían de información, han sido identificados según las denominaciones actuales vigentes, asignando a cada ejemplar su correspondiente nomenclatura actualizada.

En todos los casos para la asignación taxonómica de los ejemplares se han seguido los criterios de Salvador \& Pleguezuelos (2002) y Pleguezuelos et al. (2002). Si bien se ha actualizado la nomenclatu- 
ra según la lista patrón recientemente elaborada por la Asociación Herpetológica Española, bajo la coordinación de Montori \& Llorente (2005).

\section{LOCALIZACIÓN GEOGRÁFICA}

Se ha precisado lo más posible la posición geográfica exacta del lugar de colecta de los ejemplares. Ej., Sótano del Centro Nacional Sanidad Ambiental, M-515 km 2'3, Majadahonda. Este proceso ha diferido según la información inicial con la que hemos contado: (1) Cuando la localidad se indicaba en algún soporte (etiqueta o ficha antigua o en algún libro de entradas, registro, etc.) y era coincidente con la denominación actual, se localizaba en los mapas actuales consultados anotándose también la provincia, comunidad autónoma, etc. a la que pertenecía el lugar en cuestión. (2) Cuando el lugar había cambiado de nombre. Ej., Manjirón (=Puentes Viejas) se han actualizado los mismos, indicándose detrás de la denominación actual, entre paréntesis y antecedidas del signo "=", la denominación original del colector; seguidamente se indican las entidades geográficas mayores como en el caso anterior. (3) Cuando algún lugar de colecta o localidad ha sido imposible de localizar en los mapas actuales disponibles éstas denominaciones se han mantenido incluidas entre signos de interrogación. Ej., ¿Valtravieso? (El Pardo), Madrid.

Para la determinación de las poblaciones, parajes, etc. se ha empleado las siguientes fuentes de consulta: Euro-Atlas de Carreteras De España y Portugal (1998), Gran Atlas de Carreteras de España y Portugal (1992), Microsoft (2001), Ministerio de Fomento (2002), Nomenclator Comercial de los Pueblos de España (1992), Servicio Geográfico del Ejército (1992). Aunque el esfuerzo que hemos realizado para nombrar correctamente las localidades y más concretamente las entidades menores de población, parajes, etc., ha sido importante, somos conscientes de la inevitable comisión de errores, por ello rogamos a quienes detecten algún fallo en la denominación de las poblaciones, nos lo comuniquen para poderlos subsanar en las bases de datos y en posibles publicaciones posteriores.

Como aclaración final, queremos indicar que, dado que la Comunidad de Madrid es una Comunidad uniprovincial, cuando en los diferentes registros del catálogo aparece el término Madrid, en el campo localidad, nos referimos única y exclusivamente al municipio de Madrid capital. Ya que por repetitivo, se obvia que todas las localidades o parajes pertenecen a la provincia de Madrid y a la Comunidad de Madrid.

\section{COORDENADAS UTM}

Aunque los primeros pasos para precisar la distribución de las diferentes especies sobre la faz de la tierra no comienzan hasta bien entrado el siglo XIX (Martínez-Rica, 1997) y en la herpetología española quien realiza esos primeros intentos es Eduardo Boscá $(1877,1881)$, el panorama actual es bien diferente. La corología y la información geográfica asociada a los ejemplares colectados o visualizados tiene cada vez más importancia no sólo para elaborar mapas o atlas de distribución de especie -por ejemplo Pleguezuelos et al. (2002)sino también para su utilización en trabajos de impacto ambiental, conservación, etc. (MartínezSolano \& González-Fernández, 2003).

Por ello hemos considerado oportuno explicar en un apartado específico los diferentes pasos que se han seguido para obtener las coordenadas UTM (Universal Transversal Mercator) que acompañan a los datos de los ejemplares incluidos en este trabajo.

La mayoría de las UTM aportadas en este trabajo son meramente aproximativos y deben considerarse como orientativos. Los especialistas que empleen esta información en sus trabajos, sin duda tomarán con la debida prevención los mismos y sabrán aplicar las oportunas correcciones cuando alguno de ellos "no cuadre" en sus mapas de distribución. Pese a todo ello, creemos que pueden ahorrar muchas horas de consulta en los mapas correspondientes.

Para la determinación de dichas coordenadas se han empleado los mapas 1:50.000 del Servicio Geográfico del Ejército (SGE) en su edición de 1992, cuya proyección corresponde al elipsoide Hayford. Según cada caso, para su determinación se han establecido las siguientes normas:

1.- Cuando los datos de colecta son tan precisos que indican un paraje concreto, se indican las coordenadas de la cuadrícula de $1 \times 1 \mathrm{~km}$, en la que se encuentra situado dicho punto de colecta.

2.- Cuando las etiquetas indican sólo el nombre del municipio, ante la imposibilidad de conocer el lugar exacto del término municipal donde fue colectado, se decidió indicar la coordenada 1x1 $\mathrm{km}$ en la que se encuentra situado el centro geográfico del casco urbano del mismo (indicado en los mapas del SGE, con un triángulo y una cruz), independientemente de la extensión y posición real de dicho municipio en el término municipal.

3.- Cuando se trata de una entidad política de orden menor, la coordenada UTM que se indica también es la de la cuadrícula de $1 \times 1 \mathrm{~km}$ donde se 
encuentra ubicado el casco urbano de la aldea, pedanía, etc.

4.- Finalmente en aquellas citas que por ser tan generales son imposibles de ubicar (Ej. Zona Sur de Madrid) o bien ocupan mas de una cuadrícula de $10 \times 10 \mathrm{~km}$, no se indica ninguna coordenada.

Creemos que la importancia, cada vez mayor, de la información geográfica y su utilidad, como parte integrante de la documentación de los ejemplares (Shaffer et al., 1998), nos hace creer que es mejor partir de una información aproximada a la realidad, y susceptible de ser transformada de forma sencilla en fuente de datos de un programa de información geográfica (p. e. IDRISI o ArcView).

Por nuestra parte, con dichas coordenadas UTM, hemos elaborado unos mapas de distribución (Figuras 1 a 24), en los que mostramos las cuadrículas representadas por los ejemplares conservados en la coleción de anfibios y reptiles procedentes de la Comunidad de Madrid; estos resultados se comparan con los obtenidos por García-París et al. (1989), ampliándose las áreas de distribución para algunas de las especies estudiadas.

\section{Altitud}

En todos los casos, los valores indicados en este campo, aún cuando no se especifique nada en el catálogo, vienen dados en metros sobre el nivel medio del mar en Alicante. Por otra parte, tres han sido los modos de actuación: (1) Cuando la altitud vino dada por el colector en la documentación que acompaña a los ejemplares, ésta se indica tal cual fue anotada por éste. (2) Cuando la altitud exacta era un dato desconocido, se decidió considerar (incluida entre corchetes "[ ]") la altitud del centro geográfico del municipio (el mismo que se empleó para calcular la coordenada UTM de la cita), y que en los mapas 1:50000 del SGE (Servicio Geográfico del Ejército, 1992) empleados, viene indicada junto al nombre del municipio. (3) Cuando se trata de una entidad geográfica (Ej.: pico, punto kilométrico, etc.) se ha anotado la altitud que figura en dicho punto en el mapa 1:50.000 de SGE.

\section{Parámetros incluidos en LOS REgistros}

En cada registro se facilita, cuando se conoce y en este orden, la siguiente información: (1) Antecedido por el acrónimo del Museo Nacional de Ciencias Naturales (MNCN), el o los números de catálogo de los ejemplares relacionados. (2) El sexo y la edad de los individuos. (3) El tipo de conservación del ejemplar mediante unas abreviaturas codificadas, que se explican más detalladamente en el epígrafe "claves utilizadas". (4) Los datos de colecta de menor a mayor entidad geográfica, indicándose el paraje, accidente geográfico, pedanía, etc. donde se ha colectado el ejemplar y seguidamente la localidad y provincia. (5) Coordenadas UTM. (6) Altitud. (7) Fecha de la colecta, en formato de ocho dígitos, dos para el día, dos para el mes y cuatro para el año (dd-mm-aaaa) (8) Colector y donante. (9) Observaciones, en las que se recogen todas aquellas circunstancias dignas de mención no reflejadas con anterioridad.

Todos los campos indicados se separan mediante un punto y guión al final de cada uno de ellos. Aquellos que no se conocen se encuentran en blanco, no apareciendo guiones, signos de interrogación ni cualquier otro símbolo para indicar su inexistencia o desconocimiento.

\section{Claves empleadas}

En el catálogo se emplean los siguientes códigos abreviados:

(A) Sexo: se indica por las abreviaturas M (macho) y $\mathrm{H}$ (hembra). Cuando se individualiza un lote, se separan los sexos mediante barras oblicuas (Ej.: $\mathrm{M} / 2 \mathrm{H} / \mathrm{M}$, que indicaría que de los 4 ejemplares catalogados, el primer número corresponde a un macho, los dos siguientes a dos hembras y el cuarto otro macho); cuando se indica el número total de ellos, sin especificar es sexo de cada individuo, se indica el número total de ellos, unidos por el signo "+" $(14 \mathrm{M}+\mathrm{H})$.

(B) Edad: se indica mediante las abreviaturas $\mathrm{Hu}$ (Huevo) Jv (juvenil) Sad (subadulto) y Ad (adulto). Como en el caso anterior se separan las edades conocidas mediante barras oblicuas (Ej.: $\mathrm{Ad} / \mathrm{Jv} / 2 \mathrm{Ad}$ ) en orden correlativo de los números de catálogo, es decir el primer número de catálogo correspondería a un adulto, el segundo a un juvenil y los dos últimos a otros dos adultos; cuando se indica el número total de ellos, sin especificar la edad de cada individuo, se indica el número total de ellos, unidos por el signo "+" (8 $\mathrm{Ad}+\mathrm{Jv})$.

(C) Forma de conservación: los ejemplares se conservan de dos maneras diferentes: en fluido o en seco. En ambos casos puede tratarse de ejemplares enteros o de alguna parte, como esqueletos, pieles, etc. Las claves que se emplean son las siguientes: (a) ECF (Ejemplar Completo en 
Fluido): Es la forma más común de conservación, se guardan los ejemplares incluidos en una solución acuosa de etanol (65-70\%) o formol (10\%). (b) EDC (Esqueleto Desarticulado Completo): se guardan los huesos obtenidos de la desarticulación completa del ejemplar. Esta puede ser natural (por cultivo bacteriano) o química (con soluciones acuosas de $\mathrm{OHNa}$ o $\mathrm{OHK}$ ), en ambos casos a temperatura ambiente o en estufa a temperatura controlada en torno a los $50^{\circ} \mathrm{C}$. Los esqueletos así conseguidos se conservan en seco. (c) EDP (Esqueleto Desarticulado Parcial): se conservan sólo algunas partes del esqueleto desarticuladas de un individuo preparadas y conservadas como en el caso anterior. (d) EAC (Esqueleto Articulado Completo): Son ejemplares en los que, tras eliminar todos los tejidos blandos, se han montado las diferentes partes de su esqueleto en su posición original. Se conservan también en seco. (e) PCF (Piel completa en fluido): Se conserva la piel del ejemplar en etanol de $70^{\circ}$. Cuando las distintas partes de un mismo ejemplar se conservan de forma diferente, se emplea el signo “+” para indicarlo (Ej.: PCF+EDP). Una información más detallada sobre formas de conservación, puede consultarse en Barreiro et al. (1994).

(D) Fecha: se emplean las siguientes claves aclaratorias: (a) Cuando se desconoce la fecha exacta de colecta pero se conoce la fecha de entrada al museo o la fecha de donación, compra, etc., o está documentada en alguna publicación, se antecede a dicha fecha el símbolo " $<$ " indicativo de que la colecta fue anterior a esa fecha conocida. Ej.: $<23-08-1978$ (colecta anterior al veintitrés de agosto de 1978). (b) Cuando algún de factor de la fecha oscila entre varios valores, se indican separados por una barra (/ ). Ej.: 05/25-05-1972 indicativo de que la colecta se realizó entre los días cinco y el veinticinco de mayo de 1972. (c) Cuando el periodo de tiempo es una estación o varias, se indica así. Ej. Prim/Ver-1979.

Por último, pese a ser una costumbre bastante común la confección de catálogos siguiendo un orden sistemático, nosotros nos hemos inclinado por un orden alfabético (cada vez más frecuente), fundamentalmente por tres motivos:

(1) Seguimos las directrices de publicaciones tan relevantes en el mundo de la Herpetología, como Amphibian Species of the World (Frost, ed. 1985, Frost, 2004) o Crocodilian, Tuatara, and Turtles species of the world (King \& Burke, ed. 1989).
(2) Por otra parte los diferentes autores tampoco coinciden a la hora de considerar un orden sistemático definido (véase por ejemplo las dos obras empleadas para la asignación taxonómica: Atlas y Libro Rojo de los Anfibios y Reptiles de España (Pleguezuelos et al., 2002) y Reptiles Españoles, Identificación, historia natural y distribución (Salvador \& Pleguezuelos, 2002) que aunque coinciden en la nomenclatura de las especias, difiere en el orden de presentación de algunas Familias y géneros dentro de estas, aplicando en unos casos orden alfabético y en otros taxonómico.

(3) Con el intento de cumplir plenamente las tres facetas que según la UNESCO definen un Museo: investigación, custodia de colecciones científicas y exhibición (esta última con dos claras componentes: una expositiva y otra didáctica), hemos pensado que una ordenación alfabética, de las diferentes categorías taxonómicas, puede favorecer la localización de la información por parte de ese público que, aunque no versado en la taxonomía, como amante de la naturaleza siente el deseo de conocer los reptiles madrileños conservados en el MNCN.

\section{Resultados}

El catálogo de los reptiles madrileños conservados en el MNCN a 31 de octubre de 2005, ha sido confeccionado de acuerdo a las especificaciones y normas indicados previamente en el apartado material y métodos y con la información básica contenida en los registros que se relaciona en el Apéndice 1. La revisión efectuada en la coleción de anfibios y reptiles del MNCN, ha permitido determinar, en esa fecha, que de los cerca de 35000 ejemplares que se conservan en esta Institución, algo más del 6,5\% (2237) fueron recolectados en la Comunidad de Madrid. Toda la información referente a estos ejemplares, se ha recogido en 1039 registros informáticos. Todos ellos están adscritos a un total de 25 especies diferentes ( 2 de ellas, no pertenecientes a la fauna madrileña, Anolis s $p$. y Chamaelo chamaeleon) y cuyo número total de ejemplares por táxones, se recoge en la tabla 1.

Actualmente se acepta que en la Comunidad de Madrid se distribuyen 24 especies de reptiles (Pleguezuelos et al., 2002; Salvador \& Pleguezuelos, 2002). Estas son: Acanthodactylus erythrurus(2), Anguis fragilis, Blanus cinereus(2), Chalcides bedriagai $^{(2)}$, Chalcides striatus, Coronella austriaca, 
Tabla 1.- Número de ejemplares de reptiles madrileños conservados en el Museo Nacional de Ciencias Naturales de Madrid.

Table 1.- Number of the specimens of reptiles from Madrid preserved in the Museo Nacional de Ciencias Naturales of Madrid.

\begin{tabular}{|c|c|}
\hline TAXON & $\mathrm{N}^{\circ}$ ejemplares \\
\hline Orden CHELONII & 5 \\
\hline Familia Bataguridae & 4 \\
\hline Mauremys leprosa & 4 \\
\hline Familia Emydidae & 1 \\
\hline Emys orbicularis & 1 \\
\hline Orden AMPHISBAENIA & 130 \\
\hline Familia Amphisbaenidae & 130 \\
\hline Blanus cinereus & 130 \\
\hline Suborden Sauria (=Lacertilia) & 1669 \\
\hline Familia Chamaeleontidae & 1 \\
\hline Chamaeleo chamaeleon & 1 \\
\hline Familia Gekkonidae & 23 \\
\hline Tarentola mauritanica & 23 \\
\hline Familia Iguanidae & 1 \\
\hline Anolis sp. & 1 \\
\hline Familia Lacertidae & 1626 \\
\hline Acanthodactylus erythrurus & 144 \\
\hline Iberolacerta cyreni & 187 \\
\hline Lacerta (Lacerta) schreiberi & 77 \\
\hline Lacerta (Timon) lepida & 129 \\
\hline Podarcis hispanica & 642 \\
\hline Podarcis muralis & 104 \\
\hline Psammodromus algirus & 226 \\
\hline Psammodromus hispanicus & 117 \\
\hline Familia Scincidae & 18 \\
\hline Chalcides bedriagai & 4 \\
\hline Chalcides striatus & 14 \\
\hline Suborden Ophidia (= Serpentes) & 433 \\
\hline Familia Colubridae & 403 \\
\hline Coronella austriaca & 14 \\
\hline Coronella girondica & 31 \\
\hline Hemorrhois hippocrepis & 1 \\
\hline Macroprotodon brevis & 3 \\
\hline Malpolon monspessulanus & 82 \\
\hline Natrix sp & 1 \\
\hline Natrix maura & 154 \\
\hline Natrix natrix & 16 \\
\hline Rhinechis scalaris & 101 \\
\hline Familia Viperidae & 30 \\
\hline Vipera latasti & 30 \\
\hline TOTAL & 2237 \\
\hline
\end{tabular}

Coronella girondica, Emys orbicularis, Hemorrhois hippocrepis, Iberolacerta cyreni(1), Lacerta (Timon) lepida, Lacerta (Lacerta) schreiberi(2), Malpolon monspessulanus, Macropotodon brevis ${ }^{(4)}$, Mauremys leprosa ${ }^{(3)}$, Natrix maura, Natrix natrix, Podarcis hispanica $^{(3)}$, Podarcis muralis, Psammodromus algirus $^{(3)}$, Psammodromus hispanicus ${ }^{(3)}$, Rhinechis scalaris, Tarentola mauritanica, Vipera latasti.

De ellas, la señalada con un uno ${ }^{(1)}$, es endémica del Sistema Central (Gredos Guadarrma y Béjar), las marcadas con un dos ${ }^{(2)}$, son endémicas de la península ibérica y las marcadas con un tres ${ }^{(3)}$, corresponden a aquellas que además de por la Península Ibérica, extienden su distribución por la zona mediterránea francesa, las señaladas con ${ }^{(4)}$ además de en la península Ibérica, extendería su distribución por el norte de Marruecos.

De las 24 especies distribuidas en la Comunidad de Madrid, 23 tienen representación entre los reptiles conservados en la coleción de anfibios y reptiles del MNCN. Tan solo de Anguis fragilis, con distribución extremadamente restringida en la Comunidad de Madrid, no se guarda ningún ejemplar en el MNCN.

Se proporcionan los mapas de distribución para cada especie estudiada, en función de los lugares de origen de los ejemplares conservados, obtenidos al trasladar las coordenadas UTM de esos lugares al Mapa UTM de 10 x $10 \mathrm{~km}$ de la Comunidad de Madrid (Figuras 1 a 24).

Según esto y teniendo en cuenta que el total cuadrículas 10 x 10 que ocupan la Comunidad de Madrid son 114, y que las cuadrículas representadas en la coleción de anfibios y reptiles son 67, aumentadas en 8 más $(+8)$, si consideramos las cuadrículas compartidas con las provincias limítrofes a la Comunidad de Madrid (Ávila, Guadalajara, Segovia y Toledo) en las que existe representación en la coleción de anfibios y reptiles. Esto supone un índice de presencia de cualquier reptiles en la Comunidad de Madrid del 57,78\% que sube ( $\uparrow$ ) hasta el 65,79 considerando las cuadrículas limítrofes con representación.

Por especies, los índices de representación con respecto a los datos aportados por García-París et al. (1989), son los siguientes: Acanthodactylus erythrurus $9(+1)$ cuadrículas representadas de 35 posibles, índice del $25,71 \%$, aumenta hasta el $(\uparrow)$ 28,57\%; se amplia su distribución en 3 cuadrículas (VK06, VK17 y VL00); Anguis fragilis, ninguna cuadrícula representada $(0 \%)$; Blanus cinereus, presente en $11(+3)$ de 43 posibles, índice del 25,58\%, $(\uparrow)$ hasta el $32,55 \%$; se amplia su distribución en 2 cuadrículas (VK10 y VK47); Chalcides bedriagai, 

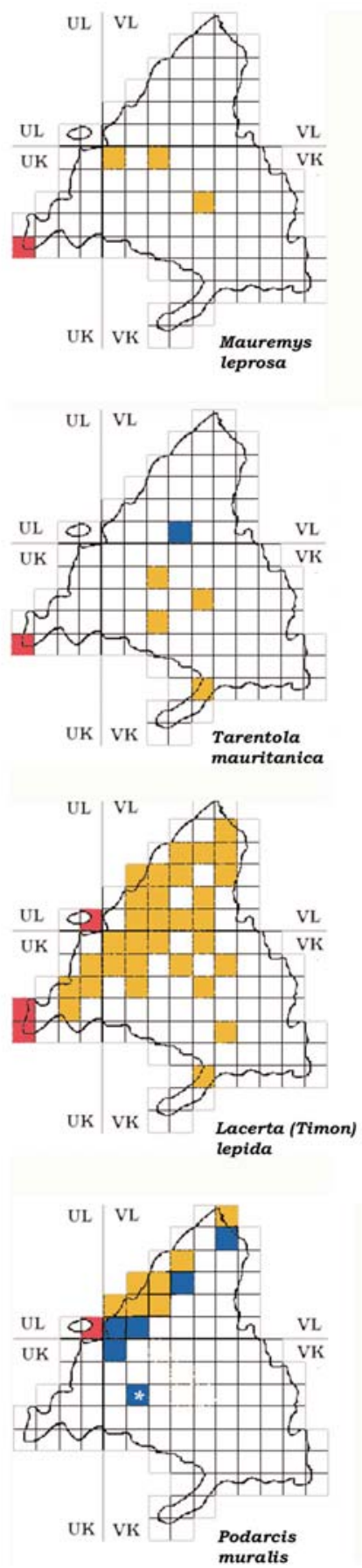
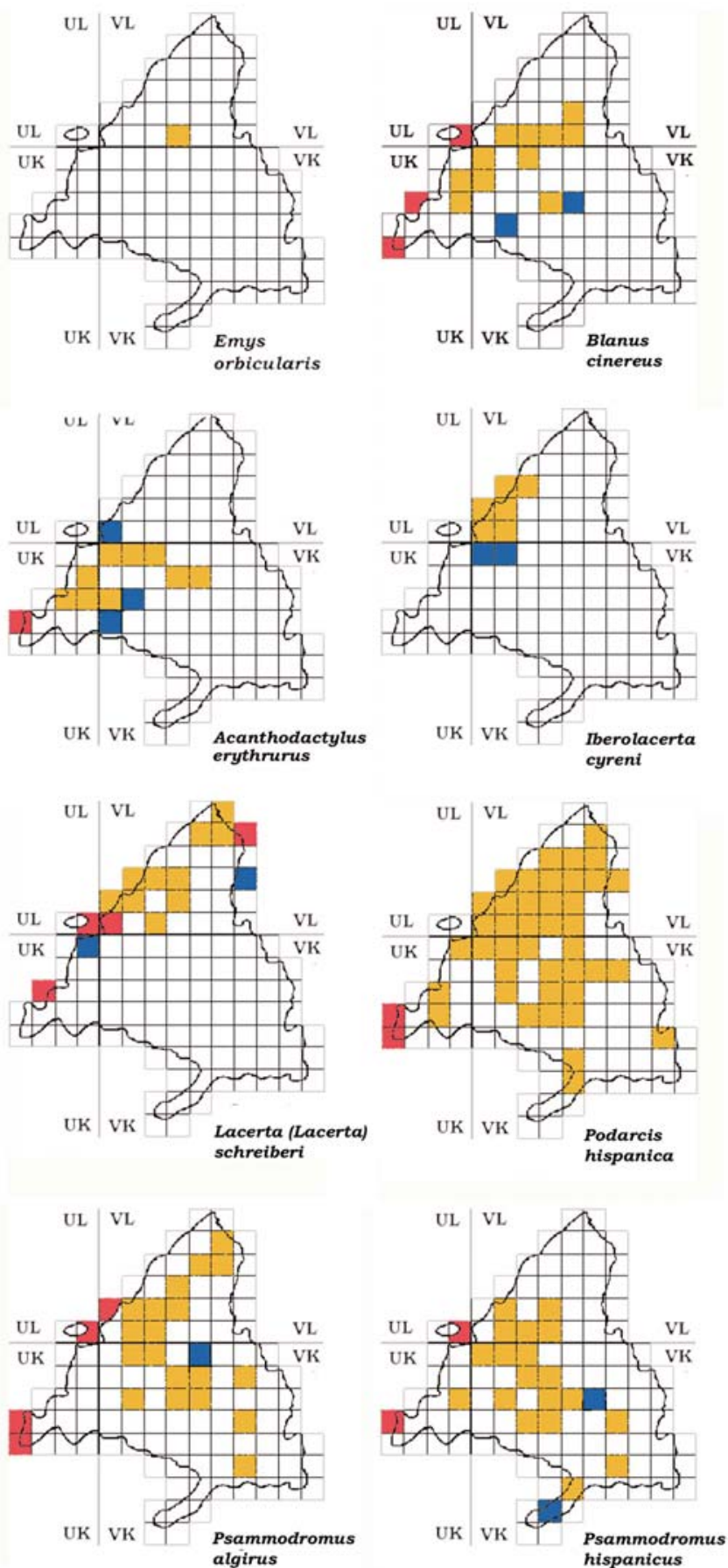

Figs. 1-24.- Mapas de distribución, por especies, de los reptiles madrileños conservados en la coleción de anfibios y reptiles (CA\&R) del Museo Nacional de Ciencias Naturales.

$\square$ Cuadrículas obtenidas de ejemplares conservados en la CA\&R del MNCN y son coincidentes con las recogidas por GarcíaParís et al. (1989).

$\square$ Cuadrículas obtenidas de ejemplares conservados en la CA\&R y coincidentes con las recogidas por García-París et al. (1989), pero colectados en las provincias limítrofes a la Comunidad de Madrid.

$\square$ Cuadrículas nuevas obtenidas de los ejemplares conservados en la CA\&R. 

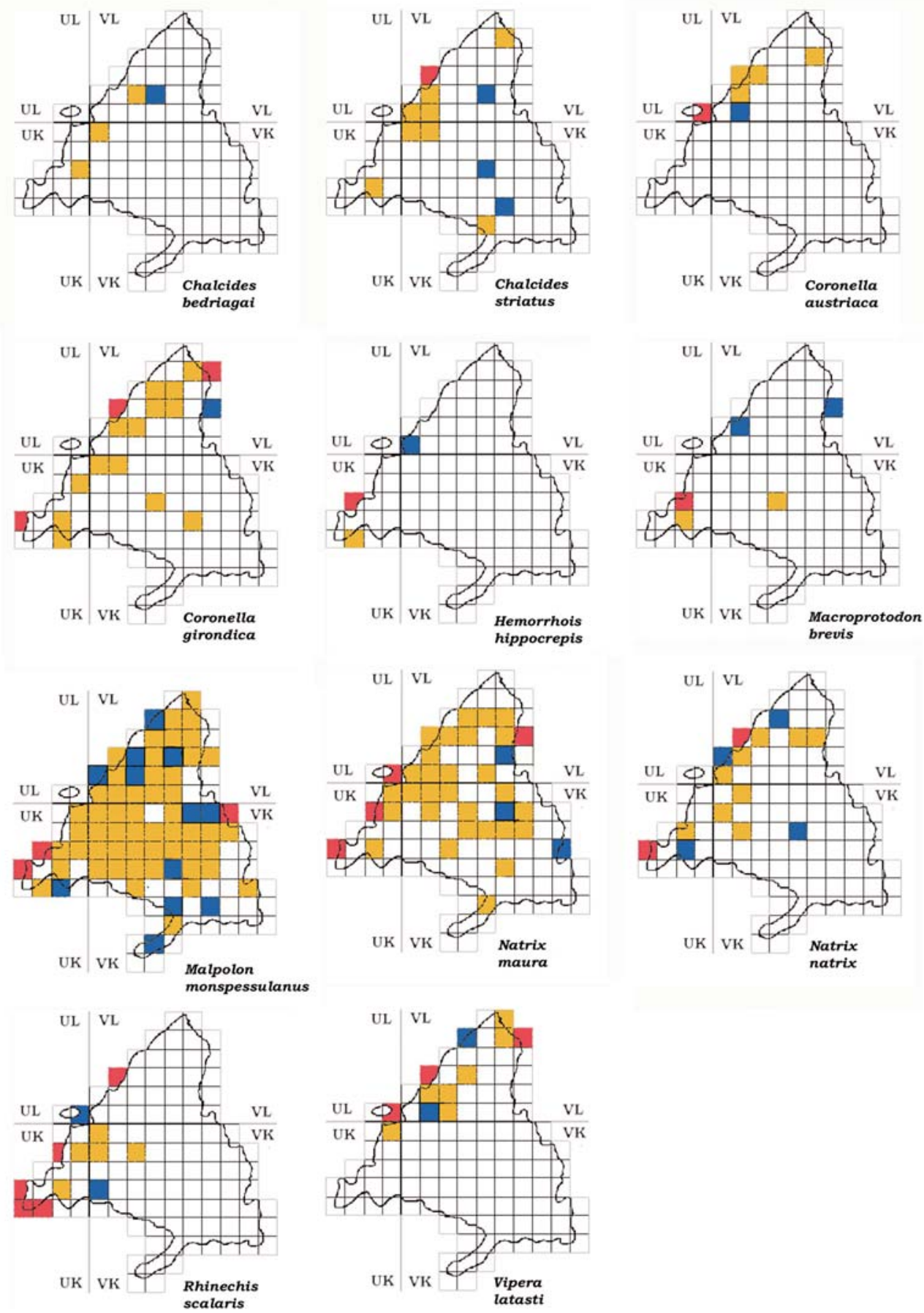

Figs. 1-24.- Distribution maps by species, of reptiles from Madrid preserved in the Collection of Amphibiasn and Reptiles (CA\&R) of the MNCN.

$\square$ Squares obtained from specimens preserved in the MNCN A\&R collection and also figured in García-París et al. (1989).

$\square$ Squares obtained from specimens preserved in the MNCN A\&R collection and also figured in García-París et al (1989) but collected in bordering provinces.

$\square$ New squares obtained from specimens preserved in the MNCN A\&R collection. 
presente en 3 de 17 posibles, 17,64\%; se amplía su distribución en 1 cuadrícula (VL31); Chalcides striatus, presente en $8(+1)$ de 33 posibles, $24,24 \%$ ( $\uparrow$ ) $27,27 \%$, se amplia su distribución en 3 cuadrículas (VK55, VK 47 y VL41); Coronella austriaca, presente en $4(+1)$ de 16 posibles, $25 \%$ ( $\uparrow) 31,25 \%$; se amplia su distribución en 1 cuadrícula (VL10); Coronella girondica, presente en $14(+3)$ de 42 posibles, 33,33\% ( $\uparrow$ ) 40,47\% se amplía su distribución en 1 cuadrícula limítrofe con Guadalajara (VL62); Emys orbicularis, presente en 1 de 9 posibles, 11,11\%; Hemorrhois hippocrepis, presente en 1 (+1) de 13 posibles, 7,69\% ( $\uparrow$ ) 15,38\%; se amplía su distribución en 1 cuadrícula (VL00); Iberolacerta cyreni, presente en 4 de 9 posibles, 44,44\%; se amplía su distribución en 2 cuadrículas (VL00 y VL10); Lacerta (Timon) lepida, presente en $31(+3)$ de 85 posibles, $36,47 \%$ ( $\uparrow$ ) 40\%; Lacerta (Lacerta) schreiberi, presente en $10(+6)$ de 24 posibles, $41,66 \%$ ( $\uparrow) 66,66 \%$; se amplía su distribución en 2 cuadrículas limítrofes con Madrid, una de Ávila (UK99) y otra de Guadalajara (VL62); Malpolon monspessulanus, presente en $54(+3)$ de 68 posibles, $79,41 \%$ ( $\uparrow) 83,82 \%$; se amplía su distribución en 11 cuadrículas (UK85, VK32, VK44, VK64, VK35, VK36, VK59, VK69, VL01, VL20, 21. VL41 y VL33); Macropotodon brevis, presente en $2(+2)$ de 9 posibles, $33,33 \%$ ( $\uparrow) 55,55 \%$, se amplía su distribución en 1 cuadrícula (VL11) y en otra cuadrícula limítrofe de Guadalajara (VL62); Mauremys leprosa, presente en $3(+1)$ de 40 posibles, 7,5\% ( $\uparrow$ ) $10 \%$; Natrix maura, presente en 27 (+5) de 69 posibles, 39,13\% ( $\uparrow$ ) 43,37\%, se amplía su distribución en 3 cuadrículas (VK58, VL51, VL33 y VL43) y en otra cuadrícula limítrofe con Guadalajara (VK86); Natrix natrix, presente en 9 (+3) de 33 posibles, $27,27 \%$ ( $\uparrow$ ) $36,36 \%$; se amplía su distribución en 3 cuadrículas (UK86, VK47 y VL33) y en una cuarta limítrofe de Segovia (VL10); Podarcis hispanica, presente en $41(+2)$ de 89 posibles, $43,06 \%$ ( $\uparrow$ ) 48,31\%; Podarcis muralis, presente en $7(+1)$ de 10 posibles, $70 \%$ ( $\uparrow$ ) $80 \%$; aunque en este caso se aumenta la distribución con respecto al trabajo de Garcia-París et al. (1989) en seis nuevas cuadrículas, una de ellas habrá que considerarla con prevención, al poderse tratarse de un posible error de datación. Se trata de los ejemplares MNCN 6767-6769 procedentes del Barranco de Cienvallejos, (Brunete). Esta cita totalmente fuera del área de distribución de la especie, y a una altitud $(580 \mathrm{~m})$, no es lógica para la especie, pero no existe duda con respecto a su correcta determinación. Muy probablemente se trata de un error de datación (aunque la etiqueta antigua es clara con respecto a los datos), pues también existen en la coleción de anfibios y reptiles un Euproctus asper (Dugès, 1852) (i!) y un Bufo bufo (Linnaeus, 1758) de la misma localidad (Cienvallejos, Brunete), de fecha (25 de septiembre de 1925) e igual colector (Cándido Bolívar). Psammodromus algirus, presente en $19(+4)$ de 72 posibles, $26,78 \%$ ( $\uparrow) 31,94 \%$, se amplía su distribución en 1 cuadrícula (VK49); Psammodromus hispanicus, presente en $19(+2)$ de 63 posibles, $30,15 \%$ ( $\uparrow$ ) $33,33 \%$, se amplía su distribución en 2 cuadrículas (VK32 yVK57); Rhinechis scalaris, presente en $5(+5)$ de 70 posibles, $7,14 \%$ ( $\uparrow) 14,28 \%$. Pese a la escasa representación geográfica, se amplía su distribución en la cuadrícula (VK06) y en otra limítrofe perteneciente a la provincia de Segovia (UL90); Tarentola mauritanica, presente en $4(+1)$ de 52 posibles, $7,69 \%(\uparrow)$ $9,61 \%$, se amplía su distribución en la cuadrícula VL30; Vipera latasti, presente en $7(+3)$ de 23 posibles, $30,43 \%$ ( $\uparrow$ ) 43,47\%; se amplía su distribución en 2 cuadrículas (VL10 y VL34). Todos estos resultados se recogen en las figuras 1 a 24 .

Como curiosidad y, como comentamos en la introducción, se conservan, dos ejemplares de dos especie de distribución foránea a la Comunidad de Madrid colectadas en las cercanías de Madrid capital: una de ellas de la franja más meridional de España y del norte de África, Chamaeleo chamaeleon, (MNCN 31065, colectado en el Cerro de los Ángeles) y otra totalmente exótica y de distribución americana, un iguánido probablemente del género Anolis (MNCN 18485, colectado en Barajas).

Finalmente indicamos que los ejemplares más antiguos que se conservan en la coleción de anfibios y reptiles del MNCN -todos ellos colectados en 1880- son: A) cuatro juveniles de Natrix maura, MNCN: 8621-8624 (colectados en Madrid por Cardiel). B) Un ejemplar también de Natrix maura, colectado en Collado Villalba por E. Boscá (MNCN 9108), C) Diez ejemplares de Podarcis hispánica, MNCN 6945 al 6954 y un ejemplar de Podarcis murales, MNCN 6944, todos ellos colectados en El Escorial, en los meses de abril y mayo, también por E. Boscá. Asimismo del siglo XIX se conservan otros ejemplares de Podarcis hispanica colectados en Madrid por E. Boscá, en junio de 1881; un macho adulto de Lacerta lepida (MNCN 6034) y otro ejemplar adulto de Rhinechis scalaris, MNCN 9050, ambos procedentes de El Escorial y colectados, respectivamente, en 1884 y 1885, por Francisco de P. Martínez y Sáez. Igualmente se conservan algunos otros ejemplares de diferentes especies, cuyas colectas están datadas entre 1886 y 1892. 


\section{Discusión y conclusiones}

Entendemos que la principal conclusión del trabajo la constituye toda la información, incluida la corológica, que se muestra en el catálogo recogido en el Apéndice 1. Aunque probablemente una parte de esta información debió ser empleada para elaborar los primeros mapas genéricos de distribución de reptiles en España (Martínez-Rica, 1989; Pleguezuelos, 1997), su escala (cuadrículas UTM de $20 \times 20 \mathrm{~km}$ ), hace que esta información, además de menos precisa, esté diluida en el conjunto de la información sobre distribución general, que querían mostrar estas obras antes mencionadas. Por ello queremos resaltar el carácter novedoso, en muchos casos, de estos datos que son mostrados, por primera vez, asociados a ejemplares concretos (y no a citas de campo) que pueden ser estudiados y analizados, pues se conservan en la colección de anfibio y reptiles del MNCN.

Por otro lado en muchos casos se ofrece información de citas históricas que pueden servir como referencia a los investigadores en la evolución de las poblaciones de reptiles, para los que los datos corológicos aportados en este trabajo pueden ser de utilidad de cara a determinar, entre otros parámetros, como afectan las agresiones al medio ambiente a la diversidad (Fisher \& Shaffer, 1996). La información facilitada, probablemente sea aún más interesante en su conjunto, si tenemos en cuenta que la única publicación específica sobre distribución de reptiles en la Comunidad de Madrid, data del año 1989 y se trata de un Atlas provisional (García-París et al., 1989), que aún no ha sido revisado y cuyos datos, en parte se corroboran aquí. Igualmente podríamos hablar tanto de la actualización de la información de ejemplares ya existentes, antes de 1989, como de nuevas incorporaciones desde entonces a la coleción de anfibios y reptiles del MNCN y, que en ambos casos, no se incluyeron en Pleguezuelos et al. (2002). Destacamos en cuanto a datos novedosos aportados por el trabajo, a un importante grupo de ejemplares (e información) que podríamos calificar de "antiguos recientes", correspondiente a todos aquellos ejemplares procedentes de la extinta Unidad de Zoología Aplicada. Colectados durante el pasado siglo, fundamentalmente durante la década de los setenta y parte de los ochenta, estos ejemplares fueron donados al MNCN por la Comunidad de Madrid en la última década del siglo XX.

Por lo que corresponde a las distribuciones geográficas deducidas de los lugares de colecta de los ejemplares conservados, el índice de representación en la coleción de anfibios y reptiles no es lo que cabría desear, si pretendemos que la muestra conservada sea realmente representativa de la diversidad natural. Esta afirmación estaría avalada por varios hechos: 1.- La representación general existente está en torno al $30 \%$ de la constatada por Garcia-París et al. (1989). 2.- En algunas especies (como Anguis fragilis), esta representación, es inexistente y en otras especies alcanza valores mínimos (Emys orbicularis, 11,11\%; Mauremys leprosa, $7,5 \%$ y Rhinechis scalaris, por otra parte una de las especies más abundantes por debajo de los $1000 \mathrm{~m}$ de altitud, y la segunda especie de ofidio con mas representantes en la coleción de anfibios y reptiles (101 ejemplares) es tan sólo del 7,14\%, lo que implica una concentración de las capturas en lugares muy concretos o estudios en lugares muy reducidos geográficamente. Por todo ello $\mathrm{y}$, pese al ligero aumento de las distribuciones conocidas (desde 1989) gracias a los datos aportados por los ejemplares conservados en la coleción de anfibios y reptiles, pensamos que por el importante desconocimiento del estado actual de muchas especies y poblaciones, es urgente el inicio de estudios que permitan actualizar y conocer en profundidad, el estado exacto de las poblaciones madrileñas de reptiles al día de hoy.

\section{AGRADECIMIENTOS}

A la Comunidad de Madrid que ha financiado este estudio, mediante el proyecto: GR/AMB/0750/2004. A Alfredo Salvador Milla y Juan Pablo Martínez Rica, que en los años setenta y principios de los ochenta del pasado siglo, por separado y con diferentes métodos, trabajaron en el intento de realizar el inventario global de la colección. A Emiliano Aguirre quien decididamente asumió como director la importancia de recuperar unas colecciones durante años olvidadas y deterioradas. A Borja Sanchiz, que a mediados de la década de los ochenta se convirtió en el impulsor de la Herpetología en el MNCN. A Rafael Márquez por la revisión del texto en inglés. A Elena Martínez-Montero y Teresa García Díez, su ayuda en la revisión de los ejemplares.

No podemos dejar de recordar, a todos aquellos que han hecho donaciones al MNCN, de los ejemplares colectados por ellos, o de su propiedad, mostrado así su amor a las colecciones y a la ciencia española. Ya sean instituciones o particulares, personas anónimas o conocidos científicos, fallecidos o contemporáneos nuestros, queremos dejar patente aquí, nuestro agradecimiento. Aunque ellos seguro que se reconocen, esperamos que los demás, también lo hagan.

1.- Institucionales.- La Comunidad de Madrid, colección de la antigua Unidad de Zoología Aplicada, referenciada como: C. A. de Madrid (UZA).

2.- Particulares.- R. Agenjo, A. Álvarez, Julio Álvarez, J. L. Amoscotegui de Saavedra, Begoña Arano, Francisco Árias, José Árias Encobet, Juan Carlos Arrechea, Baragaño, J. L. 
Bernaldo de Quirós, Juan Carlos Blanco, Ignacio Bolívar y Urrutia, Cándido Bolivar Pieltain, Eduardo Boscá y Casanoves, B. F. Bris, Stephen D. Busack, D. Caballero, Ángel Cabrera, L. Canencia, Cardiel, A. Castell, José Luis Ceresuela, Manuel Cazurro, Arturo Compte Sart, Antonio Corcuera, M. Cuesta Pons, Juan Delibes, S. Díaz, Ignacio Doádrio Villarejo, Luis Domínguez, Jesús Dorda Dorda, Benigno Elvira Payán, Rafael Escribano, Iñigo Esteban, Marisa Esteban Ruíz, A. Fernández (KRONSA), Rodrigo Fernández Fernández, Arturo Fernández Lop, J. Fernández Noniaíz, Mario García París, Antonio GarcíaValdecasas Huelin, Jesús Garzón Heydt, Paloma Garzón Heydt, Julio Gisbert de la Puente, Jose Luis González, José Enrique González Fernández, Luis Ma Gonzalez, L. M. Guio, Jeronimo. Hernández, Eduardo Hernández-Pacheco, Juan Carlos Hidalgo, Fernando F. Hiraldo, J. Hita, A. Izquierdo, Juan. Ibáñez, Sr. Krachf, A. Landín, Alberto Larramendi, javier Lobón Cerviá, B. Lorenzo, Luis Lozano Rey, Sr. Lauffer, Manuel Máñez, A. Montes, I. Marcos, Rafael Márquez Martínez de O.,Carolina Martín Albaladejo, Manuel Martínez de la Escalera, Francisco de Paula Martínez y Sáez, J. Melendro, Jesús Mellado, Srta.Millá, Eugenio Morales-Agacino, F. Morales Fortes, A. Moreno, D. Moreno, Cesar Olmos, Antonio Mujica Nava, P. Muñecas, P. Muñoz, Eugenio Ortiz, Oscar Quiroga, Fernando Palacios, A. Panadero, María de la Paz Panadero, Fernando Parra, D. Peláez, D. Pérez Imobersteg, Pérez Olea, Carlos Enrique Pérez Santos, Enrique Recio Vivar, José María Remón, Juan Carlos Rey Fraile, José Miguel Rey Salgado, T. Rico Jimeno, Luis Rivas, Arturo Rodríguez, Carlos Rodrígez Vigal, J. Roset Esteve, Jose Luis Rubio de Lucas, S. Savia, Borja Sanchiz Gil de Avalle, Óscar Soriano Hernando, Alfredo Salvador Milla, Manuel Sanz de Diego, Timoteo Sanz, J. A Suja, Rosario Rodríguez Talavera, J. Urcelay, María Valladolid Martín, J. Yerte, Antonio de Zulueta.

\section{Referencias}

AMNCN, 1868. (Archivo del Museo Nacional de Ciencias Naturales). (1868-1906). Libro de entradas y salidas de objetos de la colección de Zoología, sección Vertebrados y de Anatomía Comparada. Signatura AMNCN: CN0241/001. Madrid.

AMNCN, 1907. (Archivo del Museo Nacional de Ciencias Naturales). (1907-1913). Libro de registro de entradas de objetos a diversas secciones del Museo de Ciencias Naturales. Signatura AMNCN: CN0240/003. Madrid.

AMNCN, 1911. (Archivo del Museo Nacional de Ciencias Naturales). (1911-1932). Libro de registro de entradas de ejemplares en la colección de Zoología, sección de Osteozoología. Signatura AMNCN: CN0242/004. Madrid.

AMNCN, 1931. (Archivo del Museo Nacional de Ciencias Naturales). (1931-1939). Libro de registro de entradas de ejemplares en la colección de Zoología, sección de Osteozoología. Signatura AMNCN: CN0279/032. Madrid.

Arija, E., 1972. Geografía de España. Tomo 1. Planeta, Barcelona. 473 pp.

Barreiro, A. J., 1992. El Museo Nacional de Ciencias Naturales (1771-1935). Doce Calles. Madrid. 509 pp.
Barreiro, J., GonZÁlez-Fernández, J. E. \& Rey FrAILE, I., 1994. Las Colecciones de Vertebrados: Uso y Gestión. In: B. Sanchiz (ed). Manual de Catalogación y Gestión de las Colecciones cientificas de Historia Natural. Manuales técnicos de Museología, Vol. 1. Museo Nacional de Ciencias Naturales (CSIC). Madrid: 19-80.

Borbón y Grecia, F., 2001. Discurso de inauguración. In: J. Alonso (ed.). Actas del I Simposio sobre el Patrimonio Natural en las Colecciones Públicas de España. Diputación Foral de Navarra. Vitoria: 47-49.

Boscá, E., 1877. Catálogo de los Reptiles y Anfibios observados en España, Portugal e Islas Baleares. Anales de la Sociedad Española de Historia Natural, 6: 39-68

BoscÁ, E., 1881. Correcciones y adiciones al catálogo de los reptiles y anfibios de España Portugal y las Islas Baleares, seguido de un resumen general sobre su distribución en la Península. Anales de la Sociedad Española de Historia Natural, 10: 89-112.

Euro-ATlas DE CARRETERAS 1:300.000 DE EsPAÑA Y Portugal. 1998. Plaza \& Janés. Barcelona.

Fernández, J., GonzÁlez-Fernández, J. E., Rey, I. \& BARreiro, J., 1992. Organization and Control of Information in the Vertebrates Collections of the Museo Nacional de Ciencias Naturales. In: Gisbert et al. (eds). International Simposium and first world congress on the preservation and Conservation of natural history collections. Fundación Cultural Banesto, Comunidad Autónoma de Madrid. Madrid: 45.

Frost, D. R. (Ed.), 1985. Amphibian species of the world. A taxonomic and geographic reference. Allen Press Inc \& The Association of Systematics Collections. Lawrence. 732 pp.

Frost, D.R., 2005. World Wide Web page of the American Museum of Natural History, Department of Herpetology: "Amphibian species of the word V2.21 Database", (1998-2004). Department of Herpetology, American Museum of Natural History, New York.

García-París, M., Martín, C., Dorda, J \& Esteban, M., 1989. Atlas provisional de los anfibios y reptiles de Madrid. Revista Española de herpetología, [1988], 3(2): 237-257.

García Ballesteros, A., Méndez, R. \& Pozo, E., 1991. Parte 3 Madrid. En: Geografía de España, Vol. 7. Planeta. Barcelona: 336-589.

GonzÁlez-Fernández, J. E., 1997. La Colección de Batracología y Herpetología del Museo Nacional de Ciencias Naturales (CSIC) Madrid. Graellsia, 53: 95-100

GonzÁlez-Fernández, J. E., 2002. Colección de Anfibios y Reptiles. In: Catálogo de las Colecciones Zoológicas de Asia del Museo Nacional de Ciencias Naturales, III: Vertebrados. Manuales técnicos de 
Museología (Vol. 13). Museo Nacional de Ciencias Naturales (CSIC). Madrid: 61-180.

Graells, M. P., 1846. Catálogos de las colecciones de Anatomía Comparada, Mamíferos, Aves, Reptiles y Peces del Museo Nacional de Ciencias Naturales de Madrid. Manuscrito, Inédito, Madrid. Signatura AMNCN (Archivo del Museo Nacional de Ciencias Naturales): CN0247/001.

Gran Atlas DE CARreteras 1:200.000, 1:300.000 DE EsPaña Y PorTugal. 1992. Planeta, Barcelona.

KInG, F. W. \& Burke, R. L., 1989. Crocodilian, Tuatara, and Turtles Species of the world. A taxonomic and geographic reference. The Association of Systematics Collections. Lawrence. 216 pp.

Martínez-Rica, J. P., 1989. El Atlas provisional de los anfibios y reptiles de España y Portugal (APAREP) Presentación y situación actual. Monografías de Herpetología, 1. Asociación Herpetológica Española. Salamanca. 73 pp.

Martínez Solano, I. \& GonzÁlez Fernández, J. E., 2003. La colección de anfibios de Madrid del Museo Nacional de Ciencias Naturales y su utilidad en conservación. Graellsia, 59(2-3): 105-128.

Microsoft. 2001. Atlas Mundial Encarta 2001. 2 discos compactos (CD-Rom). Microsoft, USA.

Ministerio DE Fomento. 2002. Mapa Oficial de Carreteras. Edición 37, Interactivo V 7.0, 1 disco compacto (CD-Rom). Madrid.

Montori, A. \& Llorente, G. A. (Coord.). 2005. Lista patrón actualizada de al herpetofauna española: Conclusiones de nomenclatua y taxonomía para las especies de anfibios y reptiles de España. Actualización del Atlas y libro Rojo de los Anfibios y Reptiles de España. Dirección General de Conservación de la Naturaleza - Asociación Herpetológica Española. Madrid. 46 pp.
Nomenclator Comercial de los Pueblos de España. 1992. 14 a edición. Madrid. Imprenta Fareso. Madrid. $1040 \mathrm{pp}$.

Pleguezuelos, J. M. (ed.). 1997. Distribución y Biogrografía de los anfibios y reptiles de España y Portugal. Monografías Tierras del Sur. Universidad de Granada y Asociación Herpetológica Española. Granada: 37-44.

Pleguezuelos, J. M., Márquez, R. \& Lizana, M. (eds.). 2002. Atlas y libro Rojo de los Anfibios y Reptiles de España. Dirección General de Conservación de la Naturaleza - Asociación Herpetológica Española. Madrid. 585 pp.

RIVAS-MARTínEZ, S., 1987. Introducción: nociones sobre fitosociología biogeografía y bioclimatología. In: M. Peinado \& S. Rivas-Martínez (eds). La vegetación de España. Servicio de Publicaciones de la Universidad de Alcalá de Henares. Madrid: 19-45.

Salvador, A. \& Pleguezuelos, J. M., 2002. Reptiles Españoles, Identificación, historia natural y distribución. Canseco. Talavera de la Reina. 493 pp.

Servicio Geográfico Del EjÉrcito. 1992. Cartografía militar de España. Mapa General, Serie L, Escala 1:50.000.

Shaffer, H. B., Fisher, R. N. \& Davidson, C., 1998. The role of natural history collections in documenting species declines. Trends in Ecology and Evolution, 13(1): 27-30. 
Apéndice 1.- Catálogo de los reptiles procedentes de la Comunidad de Madrid, conservados en la coleción de anfibios y reptiles del MNCN de Madrid.

Appendix 1.- Catalogue of the reptiles from Comunidad de Madrid preserved in the Amphibian and reptile collections of the MNCN of Madrid.

\section{Clase REPTILIA}

Orden Chelonii Latreille, 1800

Familia Bataguridae Gray, 1869

Mauremys leprosa (Schweigger, 1812)

MNCN 9327.- Jv.- ECF.- San Agustín de Guadalix.- UTM: VL/4703. [684]m.- 13-05-1984.- Colectó y donó: R. R. Talavera \& J. C. Arrechea

MNCN 39230 - 39231.- 2Jv.- ECF.- Embalse de los Peñascales, Torrelodones.- UTM: VK/2392.- [740-760]m.- 01-10-1974.- Donó: C. A. de Madrid (U.Z.A.).

MNCN 41017.- Ad.- EDC - La Granjilla, El Escorial.- UTM: VK/0692 [850]m.- 11-09-1989.- Colectó y donó: J. Dorda, M. Esteban \& M. Ga París.

Familia Emydidae Refinesque, 1815

Emys orbicularis (Linnaeus, 1758)

MNCN 4108.- ECF.- R. Manzanares cruce con M-607 (=Ctra. de Colmena Viejo a Manzanares el Real), Colmenar Viejo-Manzanares el RealUTM: VL/3004.- [840]m.- 11-05-1977.- Colectó y donó: J. Urcelay.

Orden Squamata Oppel, 1811

Suborden Amphisbaenia Gray, 1844

Familia Amphisbaenidae Gray, 1844

Blanus cinereus (Vandelli, 1797)

MNCN 8122 - 8138.- 12Ad+5Jv.- ECF.- ¿Robledo?, El Escorial.- UTM: VK/08.- 05-1910.

MNCN 8161 - 8164.- 2Ad+2Jv.- ECF.- Torrelodones.- UTM: VK/2192. $[845] \mathrm{m}$.

MNCN 8174 - 8179.- 6Ad.- ECF.- El Escorial.- UTM: VK/0493.- [909]m.$<$ [09-07-1914].- Colectó: Sr. Sanz.- Donó: E. Boscá

OBSERVACIONES: Recogidos en el «Libro de registro de entradas de ejemplares en la colección de Zoología, sección de Osteozoología, 19111932» pp 67, (AMNCN, 1911).

MNCN 8180.- Ad.- ECF.- Madrid.- UTM: VK/47.- [655]m.- 05-1906.Donó: L. Lorenzo.

MNCN 8234.- Ad.- ECF.- Collado-Villalba.- UTM: VK/1699.- [917]m.25-06-1980.

MNCN 8237.- Ad.- ECF.- Alpedrete.- UTM: VL/1301.- [919]m.- 27-05-1980.

MNCN 9213.- Ad.- ECF.- Embalse de El Vellón, Guadalix de la Sierra.UTM: VL/41.- [828]m. 28-04-1975.- Colectó y donó: E. Ortiz.

MNCN 9214.- Ad.- ECF.- Navacerrada.- UTM: VL/1409.- [1203]m.- 2405-1980.

MNCN 9310.- Ad.- ECF.- Madrid.- UTM: VK/47.- [655]m.- Colectó: J. Álvarez.

MNCN 9367 - 9376.- 10Ad.- ECF.- Alpedrete.- UTM: VL/1301.- [919]m.02-04-1985.- Colectó y donó: J. Dorda, M. Esteban, C.Martín \& M García París.

MNCN 9377 - 9383.- 7Ad.- ECF.- Navalagamella.- UTM: VK/0580.[753]m.- 20-04-1985.- Colectó y donó: J. Dorda \& M. L. Esteban.

MNCN 9386.- Ad.- ECF.- San Agustín de Guadalix.- UTM: VL/4703. [684]m.- Colectó y donó: J. Dorda \& M. L. Esteban.

MNCN 9390 - 9391.- 2Ad.- ECF.- San Agustín de Guadalix.- UTM VL/4703.- [684]m.- 17-04-1983.- Colectó y donó: R. R. Talavera \& J. C. Arrechea.

MNCN 9392 - 9418.- 28(Ad+Jv).- ECF.- San Agustín de Guadalix.- UTM: VL/4703.- [684]m.- 03/04-05-1982.- Colectó y donó: R. R. Talavera \& J. C. Arrechea.

MNCN 9419.- Ad.- ECF.- San Agustín de Guadalix.- UTM: VL/4703.[684]m.- 12-05-1985.- Colectó y donó: R. R. Talavera \& J. C. Arrechea

MNCN 11842.- Ad.- PCF.- San Agustín de Guadalix.- UTM: VL/4703.[684]m.- 20-03-1983.- Colectó y donó: R. R. Talavera.

MNCN 16532.- EDC.- < [09-11-984].- Colectó y donó: J. Dorda.

MNCN 16533.- Ad.- PCF+EDP, San Agustín de Guadalix.- UTM: VL/4703.- [684]m.- 20-03-1983.- Colectó y donó: R. R. Talavera.

MNCN 24381.- Ad.- ECF.- Urbanización el Berrocal Fase 4, Becerril de la Sierra.- UTM: VL/1908.- [1020-1050]m.- 23-05-1997.- Colectó y donó: J. Dorda.
MNCN 31067 - 31069.- 3Ad.- ECF.- Navas del Rey.- UTM: UK/9371.[709]m.- 26-05-1985.- Donó: C. A. de Madrid (U.Z.A.).

MNCN 31070 - 31073 - 4Ad- ECF- Embalse de los Peñascales, Torrelodones.- UTM: VK/2392.- [740-760]m.- 11-05-1974.- Donó: C. A. de Madrid (U.Z.A.)

MNCN 31074 - 31075.- 2Ad.- ECF.- Colmenar Viejo.- UTM: VL/3501.[883]m.- 28/25-03-1970.- Donó: C. A. de Madrid U.Z.A.).

MNCN 31076 - 31079.- 4Ad.- ECF.- Embalse de los Peñascales, Torrelodones.- UTM: VK/2392.- [740-760]m.- 16-06-1973.- Donó: C. A. de Madrid (U.Z.A.)

MNCN 31082.- Ad.- ECF.- Embalse de los Peñascales, Torrelodones.UTM: VK/2392.- [740-760]m.- 13-05-1975.- Donó: C. A. de Madrid (U.Z.A.).

MNCN 31083 - 31084.- 2Ad.- ECF.- Embalse de los Peñascales, Torrelodones.- UTM: VK/2392.- [740-760]m.- 17/18-06-1975.- Donó: C. A. de Madrid (U.Z.A.).

MNCN 31106.- Ad.- ECF.- Vallecas, Madrid.- UTM: VK/4770.- [627]m.-04-1984.- Donó: C. A. de Madrid (U.Z.A.)

MNCN 31107 - 31109.- 3Ad.- ECF.- Embalse de los Peñascales, Torrelodones.- UTM: VK/2392.- [740-760]m.- 20-05-1973.- Donó: C. A. de Madrid (U.Z.A.).

MNCN 31110.- Ad.- ECF.- Embalse de los Peñascales, Torrelodones.UTM: VK/2392.- [740-760]m.- 24-06-1973.- Donó: C. A. de Madrid (U.Z.A.)

MNCN 31117 - 31122.- 6Ad.- ECF.- Navas del Rey.- UTM: UK/9371.[709]m.- -05-1985.- Donó: C. A. de Madrid (U.Z.A.).

MNCN 31123 - 31124.- 2Ad.- ECF.- Colmenar Viejo.- UTM: VL/3501.[883]m.- 01-05-1974.- Donó: C. A. de Madrid (U.Z.A.).

MNCN 31125.- Ad.- ECF.- Casa de Campo, Madrid.- UTM: VK/37.- [600650]m.- 26-05-1979.- Donó: C. A. de Madrid (U.Z.A.).

MNCN 31126.- Ad.- ECF.- Casa de Campo, Madrid.- UTM: VK/37.- [600650]m.- 19-03-1982.- Donó: C. A. de Madrid (U.Z.A.).

MNCN 31127 - 31128.- 2Ad.- ECF.- Aravaca, Madrid.- UTM: VK/3379.[647]m.- 23-06-1969.- Donó: C. A. de Madrid (U.Z.A.).

MNCN 31129 - 31132.- 4Ad.- ECF.- El Escorial.- UTM: VK/0493.[909]m.- 21-02-1970.- Donó: C. A. de Madrid (U.Z.A.).

MNCN 31133.- Ad.- ECF.- Manzanares El Real.- UTM: VL/2708.[908]m.- 17-06-1978.- Donó: C. A. de Madrid (U.Z.A.).

MNCN 31134.- Ad.- ECF.- San Agustín de Guadalix.- UTM: VL/4703.[684]m.- 28-04-1974.- Donó: C. A. de Madrid (U.Z.A.)

MNCN 31135.- Ad.- ECF.- Hoyo de Manzanares.- UTM: VK/2397.[1001]m.- 07-06-1971.- Donó: C. A. de Madrid (U.Z.A.).

MNCN 31136.- Ad.- ECF.- Moralzarzal.- UTM: VL/1704.- [979]m.- 12 05-1972.- Donó: C. A. de Madrid (U.Z.A.).

MNCN 31140.- Ad.- ECF.- Robledo de Chavela.- UTM: UK/9584.[903]m.- 01-06-1984.- Donó: C. A. de Madrid (U.Z.A.).

MNCN 31141.- Ad.- ECF.- Embalse de los Peñascales, Torrelodones.UTM: VK/2392.- [740-760]m.- 16-04-1972.- Donó: C. A. de Madrid (U.Z.A.).

MNCN 31381.- Ad.- ECF.- Embalse de los Peñascales, Torrelodones.- UTM: VK/2392.- [740-760]m.- 12-05-1974.- Donó: C. A. de Madrid (U.Z.A.). MNCN 37790.- Ad.- ECF.- Casa de Campo, Madrid.- UTM: VK/37.- [600650]m.- 22-06-1975.- Donó: C. A. de Madrid (U.Z.A.).

Suborden Lacertilia Owen, 1842

Familia Gekkonidae Oppel, 1811

Tarentola mauritanica (Linnaeus, 1758)

MNCN 4229.- Ad.- ECF.

MNCN 4774.- Ad.- ECF.- Madrid.- UTM: VK/47.- [655]m.- <[13-031928].- Colectó: C. Bolívar.

OBSERVACIONES: Pese a tener $\mathrm{n}^{\circ}$ de entrada 1928.03.03.01, no está recogido en el «Libro de registro de entradas de ejemplares en la colección de Zoología, sección de Osteozoología, 1911-1932» pp 193, por no existir anotaciones, en dicho libro, entre el 15 de diciembre de 1927 y el 8 de mayo de 1928, (AMNCN, 1911). 
MNCN 9228.- Ad.- ECF.- Madrid.- UTM: VK/47.- [655]m.- -1888.Colectó: I. Bolívar

OBSERVACIONES: Recogido en el «Libro de registro de entradas de ejemplares en la colección de Zoología, sección de Osteozoología, 19111932» pp 71, (AMNCN, 1911).

MNCN 9238.- Ad.- ECF.- Aranjuez.- UTM:VK/4832.- [494]m.- 09-091978.- Colectó: R. Fernández.

MNCN 9453.- Jv.- ECF.- Las Matas, Las Rozas de Madrid.- UTM VK/2489.- [750]m.- 23-10-1986.- Colectó: I. Marcos.

MNCN 9457.- Ad.- ECF.- C/ Pinar $n^{\circ} 18$, Madrid.- UTM: VK/4176.[650]m.- 13-11-1961.- Colectó: J. Álvarez.

MNCN 15575.- Jv.- ECF.- Jardín del MNCN, Madrid.- UTM: VK/4177.[656]m.- 18-10-1991.- Colectó y donó: S. Díaz.

MNCN 15860.- Jv.- ECF.- Patio de Zoología del MNCN, Madrid.- UTM: VK/4177.- [656]m.- 07-1985.- Colectó y donó: A. Corcuera.

MNCN 19075.- Jv.- ECF.- Patio de Zoología del MNCN, Madrid.- UTM: VK/4177.- [656]m.- 25-02-1994.- Colectó y donó: J. Dorda.

MNCN 20564.- Ad.- EDC.- Patio de Zoología del MNCN, Madrid.- UTM: VK/4177.- [656]m.- 04-05-2001.- Colectó y donó: E. Recio Vivar.

MNCN 21671.- Ad.- ECF.- Museo Nacional de Ciencias Naturales, Madrid.UTM: VK/4177.- [656]m.- 04-09-1996.- Colectó y donó: O. Quiroga.

MNCN 23317.- Jv.- ECF.- Museo Nacional de Ciencias Naturales, Madrid UTM: VK/4177.- [656]m.- 20-01-1997.- Colectó y donó: O. Soriano.

MNCN 31349 - 31352.- JV AD 3JV.- ECF.- Aranjuez.- UTM: VK/4832.[494]m.- 13/17-08-1978.- Donó: C. A. de Madrid (U.Z.A.).

MNCN 31353.- Jv.- ECF.- Parque de El Retiro, Madrid.- UTM: VK/4274.[650]m.- 25-10-1974.- Donó: C. A. de Madrid (U.Z.A.).

MNCN 31354.- Ad.- ECF.- Móstoles.- UTM: VK/2664.- [661]m.- 15-091970.- Donó: C. A. de Madrid (U.Z.A.).

MNCN 31355.- Jv.- ECF.- ¿Casa de la Mota?, Aranjuez.- UTM: VK/43.19-06-1981.- Donó: C. A. de Madrid (U.Z.A.).

MNCN 31356.- Ad.- ECF.- ¿Casa de la Mota?, Aranjuez.- UTM: VK/43.18-02-1979.- Donó: C. A. de Madrid (U.Z.A.).

MNCN 31357.- Ad.- ECF.- Madrid.- UTM: VK/47.- [655]m.- 21-071979.- Donó: C. A. de Madrid (U.Z.A.).

MNCN 31358.- Ad.- ECF.- Madrid.- UTM: VK/47.- [655]m.- 04-091972.- Donó: V (U.Z.A.).

MNCN 39776.- Ad.- ECF.- Colmenar Viejo.- UTM: VL/3501.- [883]m.$<$ [23-08-1978].- Donó: C. A. de Madrid (U.Z.A.)

MNCN 40997.- EDC.- Las Matas, Las Rozas de Madrid.- UTM: VK/2489.- [750]m.- 09-02-1994.- Colectó: I. Marcos.

MNCN 41422.- JV.- ECF.- Patio de Zoología del MNCN, Madrid.- UTM: VK/4177.- [656]m.- 02-02-1999.- Colectó y donó: J. Mª Remón.

Familia Iguanidae Gray, 1827

Anolis Daudin, 1802 (sin asignación específica)

MNCN 18485.- ECF.- Barajas, Madrid.- UTM: VK/5080.- [622]m.- 03-031990.- Colectó y donó: C. Olmos.

Familia Lacertidae Oppel, 1811

Acanthodactylus erythrurus (Schinz,1833)

MNCN 6360 - 6361.- Ad / Jv.- ECF.- Collado-Villalba.- UTM: VK/1699. [917]m. -25-06-1980- Colectó y donó: J. Mellado.

MNCN 6429.- Ad.- ECF.- Robledo de Chavela.- UTM: UK/9584. [903]m.- 18-04-1968.- Colectó y donó: C. Bolívar.

MNCN 7576.- Ad.- ECF.- Robledo de Chavela.- UTM: UK/9584.[903]m.- 12-05-1934.- Colectó y donó: C. Bolívar.

MNCN 7665.- Ad.- ECF.- San Lorenzo de El Escorial.- UTM: VK/0393. [1032]m.-- 07-1892.- Colectó y donó: E. Boscá.

OBSERVACIONES: Recogido en el «Libro de registro de entradas de ejemplares en la colección de Zoología, sección de Osteozoología, 19111932» pp 71. como «A. vulgaris», (AMNCN, 1911).

MNCN 15821.- JV.- ECF.- Río Perales [por debajo del cruce con la M-510], Navalagamella.- UTM: VK/07.- [660-700]m.- 16-05-1982.- Colectó y donó: A. Mújica Nava.

MNCN 31059 - 31060.- 2Ad.- ECF.- Villanueva de la Cañada.- UTM VK/1578.- [652]m.- 28/30-05-1973.- Donó: C. A. de Madrid (U.Z.A.).

MNCN 31529 - 31535.- 6Ad.- ECF.- El Pardo, Madrid.- UTM: VK/3486. [620-720]m.- 26-05-1972.- Donó: C. A. de Madrid (U.Z.A.).

MNCN 31771 - 31772.- 2Ad.- ECF.- El Pardo, Madrid.- UTM: VK/3486 [620-720]m.- 16-07-1972.- Donó: C. A. de Madrid (U.Z.A.).

MNCN 31804 - 31818.- 16(Ad+Jv).- ECF.- El Pardo, Madrid.- UTM VK/3486.- [620-720]m.- 16-04-1972.- C. A. de Madrid (U.Z.A.).
MNCN 31819.- Jv.- ECF.- El Pardo, Madrid.- UTM: VK/3486.- [620720]m.- 10-06-1972.- Donó: C. A. de Madrid (U.Z.A.)

MNCN 31820.- Jv.- ECF.- Pantano de San Juan, San Martín de Valdeiglesias.UTM: UK/87.- [580]m.- 11-05-1975.- Donó: C. A. de Madrid (U.Z.A.)

MNCN 31821.- Ad.- ECF.- ¿El Palancar?, Ladera Norte de la Sierra de Hoyo, Hoyo de Manzanares.- UTM: VL29.- 13-08-1971.- Colectó: J. M. Gonzálesz Cano.- Donó: C. A. de Madrid (U.Z.A.).

MNCN 31822 - 31833.- 12(Ad+Jv).- ECF.- El Pardo, Madrid.- UTM: VK/3486.- [620-720]m.- 03/10-09-1971.- Donó: C. A. de Madrid (U.Z.A.).

MNCN 31834 - 31845.- 12(Ad+Jv).- ECF.- El Pardo, Madrid.- UTM: VK/3486.- [620-720]m.- 18-05-1972.- Donó: C. A. de Madrid (U.Z.A.).

MNCN 31846 - 31849.- 4Ad.- ECF.- El Pardo, Madrid.- UTM: VK/3486.[620-720]m.- 24-06-1972.- Donó: C. A. de Madrid (U.Z.A.).

MNCN 31852 - 31862.- 11(Ad+Jv).- ECF.- El Pardo, Madrid.- UTM: KK/3486.- [620-720]m.- 25-05-1972.- Donó: C. A. de Madrid (U.Z.A.)

MNCN 31863.- Jv.- ECF.- Alpedrete.- UTM: VL/1301.- [919]m.- 03-061973.- Donó: C. A. de Madrid (U.Z.A.)

MNCN 31864.- Ad.- ECF.- El Pardo, Madrid.- UTM: VK/3486.- [620720]m.- 18-09-1972.- Donó: C. A. de Madrid (U.Z.A.).

MNCN 31865 - 31866.- 2Ad.- ECF.- Hoyo de Manzanares.- UTM: VK/2397.- [1001]m.- 29-05-1974.- Donó: C. A. de Madrid (U.Z.A.).

MNCN 31867.- Ad.- ECF.- El Pardo, Madrid.- UTM: VK/3486.- [620720]m.- 07-05-1972.- Donó: C. A. de Madrid (U.Z.A.).

MNCN 31868.- JV.- ECF.- El Pardo, Madrid.- UTM: VK/3486.- [620720]m.- 20-04-1969.- Donó: C. A. de Madrid (U.Z.A.).

MNCN 31869.- Ad.- ECF.- Embalse de los Peñascales, Torrelodones.UTM: VK/2392.- [740-760]m.- 24-06-1973.- Donó: C. A. de Madrid (U.Z.A.).

MNCN 31871 - 31876.- 6Ad.- ECF.- El Pardo, Madrid.- UTM: VK/3486.[620-720]m.- 04/05-08-1973.- Donó: C. A. de Madrid (U.Z.A.).

MNCN 31877 - 31883.- 5Ad+2Jv.- ECF.- El Pardo, Madrid.- UTM: VK/3486.- [620-720]m.- 13-04-1972.- Colectó: F. Palacios.- Donó: C. A. de Madrid (U.Z.A.)

MNCN 31884 - 31899.- 16(Ad+Jv).- ECF.- El Pardo, Madrid.- UTM: VK/3486.- [620-720]m.- 23-06-1972.- Donó: C. A. de Madrid (U.Z.A.).

MNCN 31900 - 31905.- 5Jv/Ad/Jv.- ECF.- El Pardo, Madrid.- UTM: VK/3486.- [620-720]m.- 30-09-1972.- Donó: C. A. de Madrid (U.Z.A.).

MNCN 31906 - 31912.- 7Ad.- ECF.- El Pardo, Madrid.- UTM: VK/3486.[620-720]m.- 08-05-1972.- Donó: C. A. de Madrid (U.Z.A.).

MNCN 31913 - 31914.- 2Jv.- ECF.- El Pardo, Madrid.- UTM: VK/3486.[620-720]m.- 01-10-1971.- Donó: C. A. de Madrid (U.Z.A.).

MNCN 31915.- Jv.- ECF.- Embalse de los Peñascales, Torrelodones.- UTM: VK/2392.- [740-760]m.- 14-09-1973.- Donó: C. A. de Madrid (U.Z.A.).

MNCN 31916.- Ad.- ECF.- Pantano de San Juan, San Martín de Valdeiglesias.- UTM: UK/87.- [580]m.- Verano-1975.- Donó: C. A. de Madrid (U.Z.A.)

MNCN 31917.- Ad.- ECF.- Villamantilla.- UTM: VK/0466.- [551]m.- 0506-1980.- Donó: C. A. de Madrid (U.Z.A.)

MNCN 31918 - 31927.- 10Ad.- ECF.- El Pardo, Madrid.- UTM: VK/3486.[620-720]m.- 15-07-1972.- Donó: C. A. de Madrid (U.Z.A.).

MNCN 31928 - 31930.- 3Ads.- ECF.- Embalse de los Peñascales, Torrelodones.- UTM: VK/2392.- [740-760]m.- 17/19-06-1975.- Donó: C.A.de Mdrid (U.Z.A.).

MNCN 37817.- Ad.- ECF.- La Moraleja, Alcobendas.- UTM: VK/4785.[700]m.- 10-05-1972.- Donó: C. A. de Madrid (U.Z.A.).

MNCN 39285 - 39286.- Ad/Jv.- ECF.- Navas del Rey.- UTM: UK/9371.[709]m.- 26-05-1985.- Donó: C. A. de Madrid (U.Z.A.).

Iberolacerta cyreni (Müller \& Hellmich, 1937)

MNCN 6382.- Ad.- ECF.- Cercedilla.- UTM: VL/1010.- [1188]m.- 31-051980.- Colectó y donó: J. Mellado.

MNCN 6403 - 6406.- 4Ad.- ECF.- Puerto de Navacerrada (Vertiente Sur), Navacerrada.- UTM: VL/1516.- (1680)m.- 03-08-1980.- Colectó y donó: J. Mellado.

MNCN 6500 - 6503.- 3Ad/Jv.- ECF.- Cercedilla.- UTM: VL/1010.[1188]m.- 17-06-1980.- Colectó y donó: J. Mellado.

MNCN 6504 - 6520.- 17Ad.- ECF.- Puerto de «Los Cotos», Rascafría.UTM: VL/1919.- [1830]m.- 26-06-1980.- Colectó y donó: J. Mellado.

MNCN 6539 - 6543.- 2Ad/Jv/2Ad.- ECF.- Navacerrada.- UTM: VL/1409.1203]m.- 20-05-1980.- Colectó y donó: J. Mellado.

MNCN 7168 - 7173.- 4Ad/Jv.- ECF.- Puerto de Navacerrada (Vertiente Sur), Navacerrada.- UTM: VL/1516.- 1800m.- 24-05-1970.- Colectó y donó: [J. M. Rey \& A. Landín]. 
MNCN 9437 - 9440.- -/2Jv/Ad.- ECF.- Puerto de «Los Cotos», Rascafría.UTM: VL/1919.- [1830]m.- 11-09-1983.- Colectó y donó: J. Dorda, M. Esteban, C.Martín \& M. García París.

MNCN 9441.- Jv.- ECF.- Hoya de Pepe Hernando, Rascafría.- UTM: VL/2121.- 1990m.- 11-07-1983.- Colectó y donó: M. García París

MNCN 9442 - 9445.- JV/Ad/JV/Ad.- ECF.- Navacerrada.- UTM: VL/1409. [1203]m.- -1983.- Colectó y donó: J. C. Blanco.

MNCN 13873.- Jv.- ECF.- Laguna de Peñalara, Rascafría.- UTM: VL/1921.- [1990]m.- 13-07-1986.- Colectó: M. García París \& C. Martín.- Donó: M. García París \& C. Martín.

MNCN 16507.- Ad.- EDC.- Puerto de Navacerrada (Vertiente Sur), Navacerrada.- UTM: VL/1516.- (1680)m.- 13-07-1980.- Colectó y donó: B. Elvira.

MNCN 33060 - 33061.- 2Ad.- ECF.- Siete Picos, Cercedilla.- UTM VL/1315.- [2138]m.- 20-06-1973.- Donó: C. A. de Madrid (U.Z.A.).

MNCN 33062 - 33063.- 2Ad.- ECF.- Siete Picos. Entre Segovia y Madrid, Cercedilla.- UTM: VL/1315.- [2138]m.- 20-06-1973.- Donó: C. A. de Madrid (U.Z.A.)

MNCN 33072.- ECF.- La Fuenfría, Cercedilla.- UTM: VL/1016. [1793]m.- 27-05-1973.- Donó: C. A. de Madrid (U.Z.A.).

MNCN 33073 - 33076.- ECF- Entre la Fuenfría y el Collado de las ¿Chivas? ${ }^{(*)}$, Cercedilla.- UTM: VL/00.- [1800]m.- 27-05-1973.- Donó: C. A. de Madrid (U.Z.A.)

OBSERVACIONES: $(*)$ Quizás quisieron referirse a Marichivas.

MNCN 33102 - 33103.- 2Ad.- ECF.- Siete Picos, Cercedilla.- UTM: VL/1315.- [2138]m.- 26-05-1974.- Donó: C. A. de Madrid (U.Z.A.).

MNCN 33104 - 33114.- 11Ad.- ECF.- Siete Picos, Cercedilla.- UTM: VL/1315.- [2138]m.- 21-06-1970.- Donó: C. A. de Madrid (U.Z.A.).

MNCN 33136 - 33149.- 14(Ad+Jv).- ECF.- Puerto de Navacerrada (Vertiente Sur), Navacerrada.- UTM: VL/1516.- (1680)m.- 10-09-1980.Donó: C. A. de Madrid (U.Z.A.)

MNCN 33150 - 33157.- 8(Ad+Jv).- ECF.- Puerto de Navacerrada (Vertiente Sur), Navacerrada.- UTM: VL/1516.- (1680)m.- 07-09-1980. Donó: C. A. de Madrid (U.Z.A.)

MNCN 33184 - 33187.- 4Ad.- ECF.- Siete Picos, Cercedilla.- UTM: VL/0711.- [2138]m.- 15-10-1972.- Donó: C. A. de Madrid (U.Z.A.).

MNCN 33245.- Ad.- ECF.- La Bola del Mundo, Navacerrada.- UTM VL/1715.- [2258]m.- 13-08-1973.- Donó: C. A. de Madrid (U.Z.A.)

MNCN 33246 - 33249.- 4Ad.- ECF.- Navacerrada.- UTM: VL/1409. [1203]m.- 13-08-1969.- Donó: C. A. de Madrid (U.Z.A.).

MNCN 33250 - 33259.- $10(\mathrm{Ad}+\mathrm{Jv})$ - ECF.- Puerto de Navacerrada (Vertiente Sur), Navacerrada.- UTM: VL/1516.- (1680)m.- 03/13-101980.- Donó: C. A. de Madrid (U.Z.A.).

MNCN 33260 - 33277.- 8(Ad+Jv).- ECF.- Puerto de Navacerrada (Vertiente Sur), Navacerrada.- UTM: VL/1516.- (1680)m.- 24-06-1980.Donó: C. A. de Madrid (U.Z.A.).

MNCN 33278 - 33289.- 12(Ad+Jv).- ECF.- Puerto de Navacerrada (Vertiente Sur), Navacerrada.- UTM: VL/1516.- (1680)m.- 13-07-1980. Donó: C. A. de Madrid (U.Z.A.)

MNCN 33290 - 33292.- 2Ad/Jv.- ECF.- Laguna de Peñalara, RascafríaUTM: VL/1921.- [1990]m.- 14-06-1970.- C. A. de Madrid (U.Z.A.).

MNCN 33293.- Ad.- ECF.- Laguna de Peñalara, Rascafría.- UTM VL/1921.- [1990]m.- 10-06-1975.- C. A. de Madrid (U.Z.A.).

MNCN 33294.- Ad.- ECF.- Laguna de Peñalara, Rascafría.- UTM: VL/1921.- [1990]m.- 14-06-1975.- C. A. de Madrid (U.Z.A.).

MNCN 33295.- ECF.- Cumbre de Peñalara, Rascafría.- UTM: VL/1921.[2430]m- 13-07-1976- C. A de Madrid (U.Z.A.)

MNCN 33296 - 33298.- ECF.- Laguna de Peñalara, Rascafría.- UTM: VL/1921.- [1990]m.- 13-07-1976.- C. A. de Madrid (U.Z.A.).

MNCN 33299 - 33306.- 8Ad.- ECF.- Laguna de Peñalara, Rascafría.UTM: VL/1921.- [1990]m.- 09-09-1979.- C. A. de Madrid (U.Z.A.).

MNCN 33307 - 33312.- 6Ad.- ECF.- Puerto de «Los Cotos» (Vertiente Sur), Rascafría.- UTM: VL/1919.- [1830]m.- 01-05-1970.- Donó: C. A de Madrid (U.Z.A.).

MNCN 33313.- Ad.- ECF.- Puerto de «Los Cotos» (Vertiente Sur), Rascafría. UTM: VL/1919.- [1830]m.- 02-05-1970.- Donó: C. A. de Madrid (U.Z.A.)

MNCN 33314 - 33326.- 13Ad.- ECF.- Refugio de Zabala en Peñalara, Rascafría.- UTM: VL/1922.- [2300]m.- 03-05-1970.- Donó: C. A. de Madrid (U.Z.A.)

MNCN 37813 - 37814.- 2Ad.- ECF- Puerto de Navacerrada (Vertiente Sur), Navacerrada.- UTM: VL/1516.- (1680)m.- 27-07-1981.- Donó: C. A. de Madrid (U.Z.A.).
MNCN 40134.- Hu.- ECF.- Laguna de los Pájaros (Peñalara), Rascafría.UTM: VL/2023.- [2178]m.- 06-1973.- Donó: C. A. de Madrid (U.Z.A.). MNCN 42094.- Jv.- ECF.- Siete Picos. Entre Segovia y Madrid, Cercedilla.UTM: VL/1315.- [2138]m.- 20-06-1973.- Donó: C. A. de Madrid (U.Z.A.).

Lacerta (Lacerta) schreiberi Bedriaga, 1878

MNCN 6122.- Ad.- ECF.- El Ventorrillo, Mancomunidad de Cercedilla y Navacerrada.- UTM: VL/1312.- [1500]m.- <[22-05-1933].- Colectó y donó: J. Hernández.

OBSERVACIONES: Recogido en el "Libro de registro de entradas de ejemplares en la colección de Zoología, sección de Osteozoología, 19311939" pp 11, (AMNCN, 1931).

MNCN 6124.- Ad.- ECF.- Estación alpina de «El «Ventorrillo», Mancomunidad Cercedilla y Navacerrada.- UTM: VL/11.- [1500]m.[21-06-1914].- Colectó y donó: C. Bolivar y J. Fernandez Noniaíz. OBSERVACIONES: Recogido en el «Libro de registro de entradas de ejemplares en la colección de Zoología, sección de Osteozoología, 19111932» pp 56. como «L. viridis», (AMNCN, 1911).

MNCN 6137.- Sad.- ECF.- Cercedilla.- UTM: VL/1010.- [1188]m.- 04-1913.

MNCN 6146.- Ad.- ECF.- [La Peñota], Cercedilla.- UTM: VL/1010.[1944]m.- <[20-07-1909].- Colectó y donó: W. Krachf.

OBSERVACIONES: Recogido en el «Libro de registro de entradas de objetos a diversas secciones del Museo de Ciencias Naturales, 1907-1913» pp. 41, (AMNCN, 1907)

MNCN 6147.- Ad.- ECF.- Cercedilla.- UTM: VL/1010.- [1188]m.- $<[11$ 09-1914].- Colectó: F. Árias.

ObSERVACIONES: Pese a tener $\mathrm{n}^{0}$ de entrada 1914.09.11.02, no esta Recogidos en el «Libro de registro de entradas de ejemplares en la colección de Zoología, sección de Osteozoología, 1911-1932» pp 69, por no existir anotaciones, en dicho libro, entre el 13 de julio y el 24 de septiembre, (AMNCN, 1911)

MNCN 6177 - 6179.- 3Ad.- ECF.- Río Lozoya, Rascafría.- UTM: VL/22.1100-1500m.- 24-05-1970.- Colectó y donó: [J. M. Rey \& A. Landín].

MNCN 6180.- Ad.- ECF.- Cercedilla.- UTM: VL/1010.- [1188]m.- 25-051920.- Colectó y donó: J. L. Bernaldo de Quirós.

MNCN 6193.- Jv.- ECF.- Cercedilla.- UTM: VL/1010.- [1188]m.- -08-1918. MNCN 6329 - 6330.- Jv/Ad.- ECF.- Puerto de La Fuenfría, Cercedilla.UTM: VL/1016.- (1796)m.- 28-06-1980.- Colectó y donó: J. Mellado.

MNCN 6333 - 6336.- 4Ad.- ECF.- Umbría de La Fuenfría, Cercedilla.- UTM: VL/11.- [1700-1800]m.- 28-06-1980.- Colectó y donó: [J. Mellado]

MNCN 6337 - 6338.- 2Ad.- ECF.- El Ventorrillo, Mancomunidad de Cercedilla y Navacerrada.- UTM: VL/1312.- [1500]m.- 30-04-1980.Colectó y donó: J. Mellado.

MNCN 6339.- Ad.- ECF.- [Siete Picos], Cercedilla.- UTM: VL/1315.[2138]m.- [12-07-1980].- Colectó y donó: J. Mellado.

MNCN 6418.- Sad.- ECF.- [Monasterio de] El Paular, Rascafría.- UTM: VL/2527.- [1159]m.- 04-08-1980.- Colectó y donó: [J. Mellado]

MNCN 7856.- Ad.- ECF.- Puerto de La Fuenfría, Cercedilla.- UTM: VL/1016.- (1796)m.- 08-06-1969.- Colectó y donó: [J. M. Rey \& A. Landín].

MNCN 9508 - 9509.- 2Ad.- ECF.- Rascafría.- UTM: VL/2528.- [1163]m.Colectó: A. Montes.

MNCN 17351.- Ad.- ECF. La Acebeda.- UTM: VL/4748 - [1275]m.- 2106-1978.- Colectó y donó: A. García-Valdecasas.

MNCN 33350.- Ad.- ECF.- Rascafría.- UTM: VL/2528.- [1163]m.- 10-051974.- Donó: C. A. de Madrid (U.Z.A.).

MNCN 33351 - 33354.- 4Ad.- ECF.- Puerto de «Los Cotos», Rascafría.UTM: VL/1919.- [1830]m.- 31-05-1970.- C. A. de Madrid (U.Z.A.)

MNCN 33355.- Jv.- ECF.- Rascafría.- UTM: VL/2528.- [1163]m.- 26-101975.- Donó: C. A. de Madrid (U.Z.A.)

MNCN 33362 - 33363.- 2Ad.- ECF.- El Chaparral, Montejo de la Sierra.UTM: VL/5850.- [1470]m.- 25-03-1975.- C. A. de Madrid (U.Z.A.).

MNCN 33364 - 33366.- 3Ad.- ECF.- El Chaparral, Montejo de la Sierra.UTM: VL/5850.- [1470]m.- 27-05-1972.- C. A. de Madrid (U.Z.A.)

MNCN 33367 - 33369.- 3Ad.- ECF.- El Chaparral, Montejo de la Sierra.UTM: VL/5850.- [1470]m.- 20-07-1972.- C. A. de Madrid C. A. de Madrid (U.Z.A.).

MNCN 33370 - 33373.- 4Ad.- ECF.- Montejo de la Sierra.- UTM: VL/5545.- [1148]m.- 20-07-1972.- Donó: C.A.de Madrid (U.Z.A.).

MNCN 33374.- Ad.- ECF.- El Chaparral, Montejo de la Sierra.- UTM: VL/5850.- [1470]m.- 06-07-1969.- Donó: C. A. de Madrid (U.Z.A.).

MNCN 33402.- Ad.- ECF.- Montejo de la Sierra.- UTM: VL/5545.[1148]m.- 06-1971.- Donó: C. A. de Madrid (U.Z.A.). 
MNCN 33403.- Ad.- ECF.- Montejo de la Sierra.- UTM: VL/5545.[1148]m.- 18-07-1978.- Donó: C. A. de Madrid (U.Z.A.)

MNCN 33404.- Ad.- ECF.- Montejo de la Sierra.- UTM: VL/5545. [1148]m.- 28-05-1970.- Donó: C. A. de Madrid (U.Z.A.).

MNCN 33405.- Ad.- ECF.- Montejo de la Sierra.- UTM: VL/5545.[1148]m.- -05-1970.- Donó: C. A. de Madrid (U.Z.A.).

MNCN 33406.- Ad.- ECF.- Montejo de la Sierra.- UTM: VL/5545. [1148]m.- 05-04-1977.- Donó: C. A. de Madrid (U.Z.A.)

MNCN 33407.- Ad.- ECF.- Montejo de la Sierra.- UTM: VL/5545.[1148]m.- 18-08-1970.- Donó: C. A. de Madrid (U.Z.A.).

MNCN 33416.- Ad.- ECF.- Canencia.- UTM: VL/3729.- [1150]m.- 24-111972.- Donó: C. A. de Madrid (U.Z.A.).

MNCN 33417.- Jv.- ECF.- Sierra de Guadarrama.- 10-12-1974.- Donó: C. A. de Madrid (U.Z.A.).

MNCN 33418.- Ad.- ECF.- Puerto de Canencia, Bustarviejo-CanenciaUTM: VL/3524.- [1600]m.- 30-04-1975.- Donó: C. A. de Madrid (U.Z.A.)

MNCN 33419.- JV.- ECF.- Puerto de Canencia, Bustarviejo-Canencia.UTM: VL/3524.- [1600]m.- 28-05-1973.- Donó: C. A. de Madrid (U.Z.A.).

MNCN 33420.- Ad.- ECF.- Cercedilla.- UTM: VL/1010.- [1188]m.- 30-051973.- Donó: C. A. de Madrid (U.Z.A.).

MNCN 33421 - 33422.- 2Ad.- ECF.- Cercedilla.- UTM: VL/1010. [1188]m.- 15-05-1973.- Donó: C. A. de Madrid (U.Z.A.)

MNCN 33423 - 33424.- Sad/Ad.- ECF.- Cercedilla.- UTM: VL/1010. [1188]m.- 14-05-1973.- Donó: C. A. de Madrid (U.Z.A.).

MNCN 33425 - 33428.- 4Ad.- ECF.- Las Dehesas, Cercedilla.- UTM: VL/1013.- [1400-1600]m.- 24-05-1973.- C. A. de Madrid (U.Z.A.).

MNCN 33429.- Jv.- ECF.- Laguna de Peñalara, Rascafría.- UTM: VL/1921.- [1990]m.- 09-09-1979.- Donó: C. A. de Madrid (U.Z.A.).

MNCN 33430.- Ad.- ECF.- Navacerrada.- UTM: VL/1409.- [1203]m.- 27 07-1981.- Donó: C. A. de Madrid (U.Z.A.).

MNCN 33438 - 33441.- 4Ad.- ECF.- Montejo de la Sierra.- UTM VL/5545.- [1148]m.- 25/26-05-1973.- Donó: C. A. de Madrid (U.Z.A.).

MNCN 33442.- Sad.- ECF.- Cercedilla.- UTM: VL/1010.- [1188]m.- 0407-1971.- Donó: C. A. de Madrid (U.Z.A.).

MNCN 33458.- Ad.- ECF.- Horcajuelo de la Sierra.- UTM: VL/5445.[1145]m.- 11-08-1973.- Donó: C. A. de Madrid (U.Z.A.).

MNCN 33518 - 33519.- Sad/Ad.- ECF.- Cercedilla.- UTM: VL/1010. [1188]m.- 02/06-05-1972.- Donó: C. A. de Madrid (U.Z.A.).

MNCN 33520 - 33521.- Ad/Sac.- ECF.- Cercedilla.- UTM: VL/1010.[1188]m.- 14-05-1976.- Donó: C. A. de Madrid (U.Z.A.).

MNCN 33522.- Ad.- ECF.- Cercedilla.- UTM: VL/1010.- [1188]m.- 28/3005-1978.- Donó: C. A. de Madrid (U.Z.A.).

MNCN 33523.- Ad.- ECF.- Cercedilla.- UTM: VL/1010.- [1188]m.- 15-071973.- Donó: C. A. de Madrid (U.Z.A.).

MNCN 33524.- JV.- ECF.- Miraflores de la Sierra.- UTM: VL/3518. [1150]m.- 15-10-1972.- Donó: C. A. de Madrid (U.Z.A.).

MNCN 33525.- Ad.- ECF.- Manzanares El Real.- UTM: VL/2708. [908]m.- 19-05-1974.- Donó: C.A.de Madrid (U.Z.A.)

MNCN 33603.- Jv.- ECF.- Cercedilla.- UTM: VL/1010.- [1188]m.- 04-071971.- Donó: C. A. de Madrid (U.Z.A.).

Lacerta (Timon) lepida Daudin, 1802

MNCN 6015 - 6016.- 2Jv.- ECF.- Madrid.- UTM: VK/47.- [655]m.Colectó: M. Cazurro.

MNCN 6022.- Jv.- ECF.- Laguna de Peñalara, Rascafría.- UTM: VL/1921. [1990]m.

MNCN 6023 - 6025.- 3Jv.- ECF.- Madrid.- UTM: VK/47.- [655]m.Colectó: E. Hernández Pacheco.

MNCN 6026.- Ad.- ECF.- Madrid.- UTM: VK/47.- [655]m.- Colectó: E. Hernández Pacheco.

MNCN 6033.- Ad.- ECF.- Madrid.- UTM: VK/47.- [655]m.

MNCN 6034.- Ad.- ECF.- El Escorial.- UTM: VK/0493.- [909]m.- < [05] -1884.- Colectó: [F. P.] Martínez [y Sáez].

OBSERVACIONES: Recogido en el «Libro de entradas y salidas de objetos de la colección de Zoología, sección Vertebrados y de Anatomía Comparada. 1868-1906» pp. 23, (AMNCN, 1868).

MNCN 6041.- Jv.- ECF.- El Pardo, Madrid.- UTM: VK/3486.- [620$720]$ m.- Colectó y donó: J. L. Bernaldo de Quirós.

MNCN 6069.- Ad.- ECF.- El Pardo, Madrid.- UTM: VK/3486.- [620720]m.- 06-08-1936.- Colectó y donó: J. L. Bernaldo de Quirós.

MNCN 6076.- Sad.- ECF.- Rascafría.- UTM: VL/2528.- [1163]m.- 24-051970.- Colectó y donó: [J. M. Rey \& A. Landín].
MNCN 6091.- Ad.- ECF.- Valdemorillo.- UTM: VK/0894.- [815]m.- 2906-1975.- Colectó y donó: A. Álvarez.

MNCN 6119.- Ad.- ECF.- El Pardo - La Zarzuela, Madrid.- UTM: VK/38.[650]m.- 21-06-1934.- Colectó y donó: J. L. Bernaldo de Quirós.

MNCN 6121.- Ad.- ECF.- El Pardo, Madrid.- UTM: VK/3486.- [620720]m.- Colectó: F. Árias.- Donó: Expedición del Museo.

MNCN 6417.- Ad.- ECF.- Rascafría.- UTM: VL/2528.- [1163]m.- 04-081980.- Colectó y donó: J. Mellado.

MNCN 7198 - 7203.- 3Ad/3Jv.- ECF.- San Lorenzo de El Escorial.- UTM: VK/0393.- [1032]m.- -1886.- Colectó: [F. P.] Martínez [y Sáez].

MNCN 7204.- Ad.- ECF.- Madrid.- UTM: VK/47.- [655]m.- -05-1907.Colectó y donó: J. Árias Encobet.

MNCN 7226.- Ad.- ECF.- Cerro de Vallecas, Madrid.- UTM: VK/4770.[720]m.- -04-1894.- Colectó: Sr. Fernández.- Donó: Sr. Fernández.

MNCN 16509.- Jv.- EDP.- San Agustín de Guadalix.- UTM: VL/4703.[684]m.- 03-05-1983.- Colectó y donó: R. R. Talavera \& J. C. Arrechea. MNCN 16510.- Jv.- EDP.- San Agustín de Guadalix.- UTM: VL/4703.[684]m.- 23-04-1983.- Colectó y donó: R. R. Talavera \& J. C. Arrechea.

MNCN 16512.- Jv.- PCF - EDP, Paredes de Buitrago.- UTM: VL/5239.[1020]m.- 04-07-1983.- Colectó y donó: R. R. Talavera \& A. Corcuera.

MNCN 17345.- Ad.- ECF.- El Escorial.- UTM: VK/0493.- [909]m.- 17-061978.- Colectó y donó: E. Ortíz.

MNCN 17346.- Ad.- ECF.- Somontes ( El Pardo ), Madrid.- UTM: VK/3584.- [580]m.- [<02-1983].- Colectó y donó: J. L. Amoscotegui de Saavedra.

MNCN 17348.- Ad.- ECF.- Navacerrada.- UTM: VL/1409.- [1203]m.- 0107-1980.- Colectó y donó: J. Mellado.

MNCN 22222.- Ad.- EDP.- Río Perales [por debajo del cruce con la M510], Navalagamella.- UTM: VK/0681.- [660-700]m.- 26-06-1983.Colectó y donó: A. Mújica Nava.

MNCN 32706.- Ad.- ECF.- Los Pozuelos, Manzanares El real.- UTM: VL/2305.- [920-980]m.- 09-05-1983.- Donó: C. A. de Madrid (U.Z.A.).

MNCN 32779.- Ad.- ECF.- El Pardo, Madrid.- UTM: VK/3486.- [620720]m.- Primaver-1972.- Donó: C. A. de Madrid (U.Z.A.).

MNCN 32780.- Jv.- ECF.- El Plantío, Madrid.- UTM: VK/3080.- [660680]m.- 25-08-1974.- Donó: C. A. de Madrid (U.Z.A.)

MNCN 32781.- Jv.- ECF.- El Pardo, Madrid.- UTM: VK/3486.- [620720]m.- 30-09-1972.- Donó: C. A. de Madrid (U.Z.A.).

MNCN 32782.- Jv.- ECF.- Navas del Rey.- UTM: UK/9371.- [709]m.- 2903-1985.- Donó: C. A. de Madrid (U.Z.A.).

MNCN 32783.- Sad.- ECF.- Horcajuelo de la Sierra.- UTM: VL/5445.1145]m.- 23-07-1973.- Donó: C. A. de Madrid (U.Z.A.)

MNCN 32784.- ECF.- Guadalix de la Sierra.- UTM: VL/4115.- [832]m.11-06-1985.- Donó: C. A. de Madrid (U.Z.A.)

MNCN 32785.- ECF.- Pantano de San Juan, San Martín de Valdeiglesias.UTM: UK/87 - [580]m - verano-1975 - Donó: C. A de Madrid (U.Z.A.)

MNCN 32786.- JV.- ECF.- Robledo de Chavela.- UTM: UK/9584.[903]m.- 04-08-1984.- Donó: C.A.de Madrid (U.Z.A.).

MNCN 32787.- Ad.- ECF.- Cerceda, El Boalo.- UTM: VL/2105.- [949]m.22-05-1983.- Donó: C.A.de Madrid (U.Z.A.).

MNCN 32788.- Ad.- ECF.- Navas del Rey.- UTM: UK/9371.- [709]m.- 3003-1985.- Donó: C. A. de Madrid (U.Z.A.).

MNCN 32789 - 32790.- ECF. - Embalse de El Vellón, Guadalix de la Sierra.UTM: VL/41.- [828]m.- 25-05-1985.- Donó: C. A. de Madrid (U.Z.A.)

MNCN 32791.- ECF.- Río Lozoya cruce con el puente de Taboada, Gargantilla del Lozoya.- UTM: VL/3032.- [1000]m.- 23-05-1976.Donó: C. A. de Madrid (U.Z.A.).

MNCN 32792.- ECF.- Rascafría.- UTM: VL/2528.- [1163]m.- 26-061979.- Donó: C. A. de Madrid (U.Z.A.)

MNCN 32793.- Ad.- ECF.- Montejo de la Sierra.- UTM: VL/5545. [1148]m.- verano-1971.- Donó: C. A. de Madrid (U.Z.A.).

MNCN 32794.- ECF.- Villanueva de la Cañada.- UTM: VK/1578.652]m.- 19-05-1974.- Donó: C. A. de Madrid (U.Z.A.).

MNCN 32795.- Ad.- ECF.- Colmenar Viejo.- UTM: VL/3501.- [883]m.01-05-1974.- Donó: C. A. de Madrid (U.Z.A.).

MNCN 32796.- Jv.- ECF.- Valdemaqueda.- UTM: UK/9085.- [872]m.- 2909-1973.- Donó: C. A. de Madrid (U.Z.A.)

MNCN 32797.- Jv.- ECF.- Navas del Rey.- UTM: UK/9371.- [709]m.- 0909-1984.- Donó: C. A. de Madrid (U.Z.A.).

MNCN 32798.- Ad.- ECF.- Los Pozuelos, Manzanares El real-- UTM: VL/2305.- [920-980]m.- 24-05-1983.- Donó: C. A. de Madrid (U.Z.A.). MNCN 32799.- Ad.- ECF.- Moralzarzal.- UTM: VL/1704.- [979]m.- 0306-1972.- Donó: C. A. de Madrid (U.Z.A.). 
MNCN 32800.- Ad.- ECF.- Cercedilla.- UTM: VL/1010.- [1188]m.- 12-051972.- Donó: C. A. de Madrid (U.Z.A.).

MNCN 32801 - 32802.- 2Ad.- ECF.- El Pardo, Madrid.- UTM: VK/3486.[620-720]m.- 24-06-1972.- Donó: C. A. de Madrid (U.Z.A.).

MNCN 32803.- Sad.- ECF.- Buitrago de Lozoya.- UTM: VL/4638. [1073]m.- 10-09-1977.- Donó: C. A. de Madrid (U.Z.A.).

MNCN 32804 - 32806.- ECF.- Navas del Rey.- UTM: UK/9371.- [709]m.20-07-1985.- Donó: C. A. de Madrid (U.Z.A.).

MNCN 32807.- Ad.- ECF.- El Pardo, Madrid.- UTM: VK/3486.- [620720]m.- 23-06-1972.- Donó: C.A.de Madrid (U.Z.A.).

MNCN 32808.- Ad.- ECF.- Navas del Rey.- UTM: UK/9371.- [709]m.- 2405-1985.- Donó: C. A. de Madrid (U.Z.A.).

MNCN 32809.- Ad.- ECF.- Granja Escuela «El Palomar», Chapinería.UTM: UK/9770.- [680]m.- 02-07-1985.- Donó: C. A. de Madrid (U.Z.A.)

MNCN 32810.- Ad.- ECF.- San Martín de la Vega.- UTM: VK/5151. [515]m.- 31-05-1977.- Donó: C.A.de Madrid (U.Z.A.).

MNCN 32811.- Ad.- ECF.- Navas del Rey.- UTM: UK/9371.- [709]m.- 0906-1984.- Donó: C. A. de Madrid (U.Z.A.)

MNCN 32812.- Jv.- ECF.- La Pedriza, Manzanares El Real.- UTM: VL/21.- [1400-1700]m.- 23-05-1974.- Donó: C. A. de Madrid (U.Z.A.)

MNCN 32813.- Jv.- ECF.- El Pardo, Madrid.- UTM: VK/3486.- [620720]m.- 12-09-1970.- Donó: C.A.de Madrid (U.Z.A.).

MNCN 32814.- Jv.- ECF.- Villanueva de la Cañada.- UTM: VK/1578.[652]m.- 27-09-1974.- Donó: C.A.de Madrid (U.Z.A.).

MNCN 32815.- Ad.- ECF.- Barajas, Madrid.- UTM: VK/5180.- [622]m.17-04-1974.- Donó: C. A. de Madrid (U.Z.A.).

MNCN 32816.- Ad.- ECF.- Los Pozuelos, Manzanares El real.- UTM: VL/2305.- [920-980]m.- 22-05-1983.- Donó: C. A. de Madrid (U.Z.A.)

MNCN 32817.- Ad.- ECF.- Navas del Rey.- UTM: UK/9371.- [709]m.- 0505-1985.- Donó: C. A. de Madrid (U.Z.A.)

MNCN 32818.- Ad.- ECF.- Alrededores de Madrid, Madrid.- 25-04-1973.Donó: C. A. de Madrid (U.Z.A.)

MNCN 32819.- Ad.- ECF.- Embalse de los Peñascales, Torrelodones.- UTM VK/2392.- [740-760]m.- 18-06-1975.- Donó: C. A. de Madrid (U.Z.A.).

MNCN 32820.- Jv.- ECF.- Embalse de los Peñascales, Torrelodones.- UTM: VK/2392.- [740-760]m.- 16-06-1973.- Donó: C. A. de Madrid (U.Z.A.).

MNCN 32821 - 32822.- Ad/Sad.- ECF.- Manzanares El Real.- UTM: VL/2708.- [908]m.- 22-06-1978.- Donó: C.A.de Madrid (U.Z.A.).

MNCN 32823.- Jv.- ECF.- Robledo de Chavela.- UTM: UK/9584. [903]m.- 04-05-1975.- Donó: C.A.de Madrid (U.Z.A.).

MNCN 32824.- Ad.- ECF.- 08-1971.- Donó: C. A. de Madrid (antigua Unidad de Zoología Aplicada).

MNCN 32825.- Ad.- ECF.- Villanueva del Pardillo.- UTM: VK/1882.[652]m.- 02-07-1975.- Donó: C.A.de Madrid (U.Z.A.).

MNCN 32826.- Ad.- ECF.- Montejo de la Sierra.- UTM: VL/5545.[1148]m.- 14-05-1973.- Donó: C.A.de Madrid (U.Z.A.).

MNCN 32827.- Ad.- ECF.- Barajas, Madrid.- UTM: VK/5180.- [622]m.15-05-1974.- Donó: C. A. de Madrid (U.Z.A.).

MNCN 32828.- Ad.- ECF.- Puerto de Canencia, Bustarviejo-Canencia.UTM: VL/3524.- [1600]m.- 30-04-1975.- Donó: C. A. de Madrid (U.Z.A.).

MNCN 32829.- Ad.- ECF.- Navas del Rey.- UTM: UK/9371.- [709]m.- 2207-1985.- Donó: C. A. de Madrid (U.Z.A.).

MNCN 32830.- Ad.- ECF.- Hoyo de Manzanares.- UTM: VK/2397. [1001]m.- 05-04-1970.- Donó: C. A. de Madrid (U.Z.A.).

MNCN 32831.- Sad.- ECF.- Villavieja de Lozoya.- UTM: VL/4339. [1066]m.- 01-06-1969.- Donó: C. A. de Madrid (U.Z.A.).

MNCN 32832.- Ad.- ECF.- Galapagar.- UTM: VK/1592.- [881]m.- 12-061973.- Donó: C. A. de Madrid (U.Z.A.).

MNCN 32833.- Ad.- ECF.- Rascafría.- UTM: VL/2528.- [1163]m.- 28-031970.- Donó: C. A. de Madrid (U.Z.A.).

MNCN 32834.- Ad.- ECF.- El Pardo, Madrid.- UTM: VK/3486.- [620720]m.- 24-06-1972.- Donó: C. A. de Madrid (U.Z.A.).

MNCN 32835 - 32836.- 2Ad.- ECF.- El Pardo, Madrid.- UTM: VK/3486.[620-720]m.- 25-05-1972.- Donó: C. A. de Madrid (U.Z.A.).

MNCN 32837.- Ad.- ECF.- Robledo de Chavela.- UTM: UK/9584.[903]m.- 09-07-1985.- Donó: C. A. de Madrid (U.Z.A.).

MNCN 32838.- Ad.- ECF.- El Pardo, Madrid.- UTM: VK/3486.- [620720]m.- 28-06-1973.- Donó: C. A. de Madrid (U.Z.A.).

MNCN 32839.- Ad.- ECF.- El Pardo, Madrid.- UTM: VK/3486.- [620720]m.- 19-07-1972.- Donó: C. A. de Madrid (U.Z.A.).

MNCN 32840.- Ad.- ECF.- Villanueva de la Cañada.- UTM: VK/1578. [652]m.- 09-04-1972.- Donó: C. A. de Madrid (U.Z.A.)
MNCN 32841.- Ad.- ECF.- Colmenar Viejo.- UTM: VL/3501.- [883]m.18-03-1970.- Donó: C. A. de Madrid (U.Z.A.).

MNCN 32842 - 32843.- 2Ad.- ECF.- Soto de Viñuelas, Tres Cantos.- UTM: VK/4295.- [660-700]m.- 23/17-06-1971.- Donó: C. A. de Madrid (U.Z.A.).

MNCN 32844.- Ad.- ECF.- Vallecas, Madrid.- UTM: VK/4770.- [627]m.14-06-1975.- Donó: C. A. de Madrid (U.Z.A.).

MNCN 32845.- ECF.- Los Pozuelos, Manzanares El real.- UTM: VL/2305.- [920-980]m.- 24-05-1983.- Donó: C. A. de Madrid (U.Z.A.).

MNCN 32846.- Ad.- ECF.- El Pardo, Madrid.- UTM: VK/3486.- [620720]m.- 17-07-1972.- Donó: C. A. de Madrid (U.Z.A.).

MNCN 32847.- JV.- ECF.- Montejo de la Sierra.- UTM: VL/5545.[1148]m.- 20-07-1972.- Donó: C. A. de Madrid (U.Z.A.)

MNCN 32848.- Ad.- ECF.- El Escorial.- UTM: VK/0493.- [909]m.- 28-051967.- Donó: C. A. de Madrid (U.Z.A.).

MNCN 32849.- Jv.- ECF.- Robledo de Chavela.- UTM: UK/9584.[903]m.- 17-06-1984.- Donó: C. A. de Madrid (U.Z.A.).

MNCN 32850.- Jv.- ECF.- Laguna de Ontígola, Aranjuez.- UTM: VK/4930.- [560]m.- 12-10-1975.- Donó: C. A. de Madrid (U.Z.A.).

MNCN 32851 - 32852.- ECF.- Navas del Rey.- UTM: UK/9371.- [709]m.15/18-04-1975.- Donó: C. A. de Madrid (U.Z.A.).

MNCN 32853.- Ad.- ECF.- Navas del Rey.- UTM: UK/9371.- [709]m.- 3103-1985.- Donó: C. A. de Madrid (U.Z.A.).

MNCN 32854.- ECF.- Finca de los Ángeles, San Martín de la Vega.- UTM: VK/5457.- [540]m.- 24-06-1973.- Donó: C. A. de Madrid (U.Z.A.).

MNCN 32855.- Jv.- ECF.- Navas del Rey.- UTM: UK/9371.- [709]m.- 1007-1985.- Donó: C. A. de Madrid (U.Z.A.).

MNCN 32856.- Ad.- ECF.- El Pardo, Madrid.- UTM: VK/3486.- [620720]m.- 12-05-1977.- Donó: C. A. de Madrid (U.Z.A.).

MNCN 32857.- Jv.- ECF.- Navas del Rey.- UTM: UK/9371.- [709]m.- 1905-1985.- Donó: C. A. de Madrid (U.Z.A.).

MNCN 32858.- Ad.- ECF.- Brunete.- UTM: VK/1573.- [656]m.- 30-071972.- Colectó: J. L. Ceresuela.- Donó: C. A. de Madrid (U.Z.A.).

MNCN 32859.- Ad.- ECF.- Finca de los Ángeles, San Martín de la Vega.UTM: VK/5457.- [540]m.-- 19-05-1973.- Donó: C. A. de Madrid (U.Z.A.).

MNCN 32860.- Jv.- ECF.- Navas del Rey.- UTM: UK/9371.- [709]m.- 1211-1984.- Donó: C. A. de Madrid (U.Z.A.).

MNCN 32861.- Ad.- ECF.- Río Lozoya cruce con el puente de Taboada, Gargantilla del Lozoya.- UTM: VL/3032.- [1000]m.- 15-06-1975.Donó: C. A. de Madrid (U.Z.A.)

MNCN 32862.- Ad.- ECF.- Ctra. M-126, Buitrago de Lozoya-Manjirón.UTM: VL/43.- [980-1070]m.- 24-05-1973.- Donó: C. A. de Madrid (U.Z.A.).

MNCN 32863.- Ad.- ECF.- Barajas, Madrid.- UTM: VK/5180.- [622]m.21-05-1975.- Donó: C. A. de Madrid (U.Z.A.)

MNCN 37836 - 36837.- 2Ad.- ECF.- Navas del Rey.- UTM: UK/9371.[709]m.- 09-05-1985.- Donó: C. A. de Madrid (U.Z.A.)

MNCN 37838 - 37840.- 2Sad.- ECF.- El Berrueco.- UTM: VL/5326.[925]m.- 03-06-1975.- Donó: C. A. de Madrid (U.Z.A.).

MNCN 37844 - 37846.- 3Ad.- ECF.- El Pardo, Madrid.- UTM: VK/3486.[620-720]m.- 26-05-1972.- Donó: C. A. de Madrid (U.Z.A.).

MNCN 41021.- Ad.- EPF - EDP, San Agustín de Guadalix.- UTM: VL/4703.[684]m.- 03-05-1983.- Colectó y donó: R. R. Talavera \& J. C. Arrechea.

MNCN 41022.- Jv.- EPF - EDP, Collado-Villalba.- UTM: VK/1699.[917]m.- 04-06-1982.

MNCN 41050.- Ad.- EDC, San Agustin de Guadalix.- UTM: VL/4703.[684]m.- 30-04-1983.- Colectó y donó: R. R. Talavera \& J. C. Arrechea.

MNCN 41508.- Ad.- ECF.- Pelayos de la Presa.- UTM: UK/8568.[570]m.- [20-10-2000].- Colectó y donó: C. Olmos.

MNCN 41509.- Ad.- ECF.- Pelayos de la Presa.- UTM: UK/8568.[570]m.- 07-08-1975.

Podarcis hispanica (Steindachner, 1870)

MNCN 6384 - 6398.- 15 Ad.- ECF.- Puerto de La Fuenfría, Cercedilla.UTM: VL/1016.- (1796)m.- 09-07-1980.- Colectó y donó: J. Mellado.

MNCN 6401.- Ad.- ECF.- Camorrito, Cercedilla.- UTM: VL/1112.[1400]m.- 03-07-1980.- Colectó y donó: J. Mellado.

MNCN 6402.- Ad.- ECF.- Cercedilla.- UTM: VL/1010.- [1188]m.- 27-061980.- Colectó y donó: J. Mellado.

MNCN 6425 - 6428.- 4Ad.- ECF.- Cercedilla.- UTM: VL/1010.- [1188]m.31-05-1980.- Colectó y donó: J. Mellado.

MNCN 6432 - 6437.- $3 \mathrm{Ad} / \mathrm{JV} / \mathrm{Ad} / \mathrm{JV}$.- ECF.- Navacerrada.- UTM: VL/1409.- [1203]m.- 21/22-05-1980.- Colectó y donó: J. Mellado. 
MNCN 6444.- As.- ECF.- Alpedrete.- UTM: VL/1301.- [919]m.- 25-051980.- Colectó y donó: J. Mellado.

MNCN 6445 - 6447.- 3Ad.- ECF.- Alpedrete.- UTM: VL/1301.- [919]m.25-06-1980.- Colectó y donó: J. Mellado.

MNCN 6458 - 6460.- 3Ad.- ECF.- Alpedrete.- UTM: VL/1301.- [919]m.27-05-1980.- Colectó y donó: J. Mellado.

MNCN 6464 - 6473.- 4Ad.- ECF.- Navacerrada.- UTM: VL/1409. [1203]m.- 01-07-1980.- Colectó y donó: J. Mellado.

MNCN 6474.- Ad.- ECF.- El Ventorrillo, Mancomunidad de Cercedilla y Navacerrada.- UTM: VL/1312.- [1500]m.- 12-07-1980. Colectó y donó: J Mellado.

MNCN 6493.- Ad.- ECF.- El Ventorrillo, Mancomunidad de Cercedilla y Navacerrada.- UTM: VL/1312.- [1500]m.- 13-07-1980.- Colectó y donó: J. Mellado.

MNCN 6495.- Ad.- ECF.- Umbría de la Fuenfría, Cercedilla.- UTM: VL/11.- [1700-1800]m.- 28-06-1980.- Colectó y donó: J. Mellado.

MNCN 6496 - 6497.- 2Ad.- ECF.- Collado-Villalba.- UTM: VK/1699.[917]m.- 25-06-1980.- Colectó y donó: J. Mellado.

MNCN 6656 - 6660.- 3Jv/2Ad.- ECF.- Río Lozoya, Rascafría.- UTM VL/22.- 1500m.- 24-05-1970.- Colectó y donó: [J. M. Rey \& A. Landín]

MNCN 6780.- Ad.- ECF.- El Escorial.- UTM: VK/0493.- [909]m.$<[1936]$.- Colectó: M. Martínez de la Escalera.

MNCN 6781 - 6782.- 2Ad.- ECF.- Alrededores de Aranjuez.- UTM: VK/4832.- [490-500]m.- <[02-1914].- Colectó y donó: F. Árias.

OBSERVACIONES: Recogido en el «Libro de registro de entradas de ejemplares en la colección de Zoología, sección de Osteozoología, 19111932» pp 45, como “L. muralis"(AMNCN, 1911).

MNCN 6783.- Ad.- ECF.- El Escorial.- UTM: VK/0493.- [909]m.

MNCN 6798.- Ad.- ECF.- Madrid.- UTM: VK/47.- [655]m.

MNCN 6799 - 6846.- 48(M+H+Jv).- ECF.- Leganés.- UTM: VK/3465.[665]m.

MNCN 6890.- Ad.- ECF.- Madrid.- UTM: VK/47.- [655]m.- -06-1881.Colectó y donó: E. Boscá.

OBSERVACIONES: Recogido en el «Libro de registro de entradas de ejemplares en la colección de Zoología, sección de Osteozoología, 1911 1932» pp 68, (AMNCN, 1911).

MNCN 6908 - 6910.- Jv/2Ad.- ECF.- El Pardo, Madrid.- UTM: VK/3486. [620-720]m.

MNCN 6912.- Sad.- ECF.- El Ventorrillo, Mancomunidad de Cercedilla y Navacerrada.- UTM: VL/1312.- [1500]m.- 1931.- Colectó y donó: E. Morales-Agacino.

OBSERVACIONES: Pese a tener $\mathrm{n}^{\circ}$ de entrada 1931.04.01.01-02, no esta Recogido en el «Libro de registro de entradas de ejemplares en la colección de Zoología, sección de Osteozoología, 1911-1932» pp 200, por no existir anotaciones, en dicho libro, entre el 7 de marzo y el 10 de abril, (AMNCN, 1911).

MNCN 6945 - 6954.- 10Ad.- ECF- El Escorial.- UTM: VK/0493. [909]m.- -04/05-1880.- Colectó y donó: E. Boscá

OBSERVACIONES: Recogidos en el «Libro de registro de entradas de ejemplares en la colección de Zoología, sección de Osteozoología, 19111932» pp 68. como «L. muralis», (AMNCN, 1911).

MNCN 7240 - 7247.- 8Ad.- ECF.- Rascafría.- UTM: VL/2528.- 1100m.Colectó y donó: [J. M. Rey \& A. Landín]

MNCN 9245.- Ad.- ECF.- Santa María de la Alameda.- UTM: UK/9394. [1460]m.- 20-10-1985.- Colectó y donó: M. García París \& C. Martín.

MNCN 9251.- Ad.- ECF.- Embalse de Valmayor, Galapagar.- UTM VK/18 - [831]m.- -06-1985.- Colectó y donó: M. García-París.

MNCN 9254.- Ad.- ECF.- Puerto de La Fuenfría, Cercedilla.- UTM: VL/1016.- (1796)m.- 04-07-1980.- Colectó y donó: J. Mellado.

MNCN 9267 - 9270.- 4Ad.- ECF.- E.T.S.I. Montes (Ciudad Universitaria), Madrid.- UTM: VK/3878.- [620]m.- 13-05-1983.- Colectó y donó: M. García París.

MNCN 9271 - 9274.- Ad/Jv/2Ad.- ECF.- San Agustín de Guadalix.- UTM: VL/4703.- [684]m.- 02-02-1985.- Colectó y donó: J. Dorda, M. Esteban, C.Martín \& M. García París

MNCN 9332 - 9335.- 4Ad.- ECF.- San Agustín de Guadalix.- UTM VL/4703.- [684]m.- 27-03-1985.- Colectó y donó: J. E. González, R. R. Talavera \& B. Sanchiz.

MNCN 9361.- Ad.- ECF.- Lozoya.- UTM: VL/3333.- [1114]m.- 04-051984.- Colectó y donó: M. García París.

MNCN 9364.- Ad.- ECF.- Guadarrama.- UTM: VL/0803.- [981]m.- 18-061970.- Colectó y donó: J. M. Rey Salgado.
MNCN 9436.- Ad.- ECF.- ¿Parque de la Colina ?, Madrid.- UTM: VK/47.05-1985.- Colectó y donó: M. Máñez.

MNCN 9549 - 9551.- 3Ad.- ECF.- Alpedrete.- UTM: VL/1301.- [919]m.02-04-1985.- Colectó y donó: J. Dorda, M. Esteban, C.Martín \& M. García París.

MNCN 9552.- Ad.- ECF.- Madrid.- UTM: VK/47.- [655]m.- 05-1960.Colectó: J. Álvarez.

MNCN 10018.- Ad.- ECF.- Ciudad Universitaria, Madrid.- UTM: VK/37.[600]m.- 06-02-1986.- Colectó y donó: M. García París.

MNCN 15861.- Sad.- ECF.- Cerro de los Angeles, Getafe.- UTM: VK/4262.- [670]m.- 09-09-1983.- Colectó: J. C. Hidalgo.

MNCN 16492.- Ad.- EDP.- San Agustín de Guadalix.- UTM: VL/4703.[684]m.- 19/20-03-1983.- Colectó y donó: R. R. Talavera.

MNCN 16493.- Ad.- EDP.- San Agustín de Guadalix.- UTM: VL/4703.684]m.- 19/20-03-1983.- Colectó y donó: R. R. Talavera.

MNCN 16494.- Ad.- EDP.- Embalse de Pinilla, Lozoya-Pinilla del Valle.UTM: VL/33.- [1098]m.- 01-05-1982.- Colectó y donó: L. Canencia

MNCN 16495.- Ad.- EAC.- Embalse de los Peñascales, Torrelodones.UTM: VK/2392.- [740-760]m.- 11-03-1983.- Colectó y donó: R. R. Talavera \& A. Corchera.

MNCN 16497.- Ad.- EDP.- San Agustín de Guadalix.- UTM: VL/4703.684]m.- 19/20-03-1983.- Colectó y donó: R. R. Talavera.

MNCN 16498.- Ad.- EDP.- San Agustín de Guadalix.- UTM: VL/4703.[684]m.- 19/20-03-1983.- Colectó y donó: R. R. Talavera

MNCN 16499.- Ad.- EDP.- San Agustín de Guadalix.- UTM: VL/4703.[684]m.- 19/20-03-1983.- Colectó y donó: R. R. Talavera.

MNCN 16500.- Ad.- EDP.- San Agustín de Guadalix.- UTM: VL/4703.[684]m.- 19/20-03-1983.- Colectó y donó: R. R. Talavera.

MNCN 16501.- Ad.- EDP.- San Agustín de Guadalix.- UTM: VL/4703.[684]m.- 19/20-03-1983.- Colectó y donó: R. R. Talavera.

MNCN 16502.- Ad.- EDC.- Embalse de los Peñascales, Torrelodones.UTM: VK/2392.- [740-760]m.- 01-03-1983.- Colectó y donó: I. Doadrio. MNCN 24200.- Ad.- ECF.- Navas del Rey.- [709]m.- 26-05-1985.

MNCN 34300 - 34301.- 2Ad.- ECF.- 01-04-1970.- Donó: C. A. de Madrid (U.Z.A.).

MNCN 34302.- Jv.- ECF.- 15-11-1979.- Donó: C. A. de Madrid (U.Z.A.)

MNCN 34303.- Ad.- ECF.- 03-04-1980.- Donó: C. A. de Madrid (U.Z.A.).

MNCN 34304.- Ad.- ECF.- M-126 (=Ctra. de Buitrago de Lozoya a, Manjirón (=Puentes Viejas).- UTM: VL/43.- [970-1070]m.- 24-051973.- Donó: C. A. de Madrid (U.Z.A.).

MNCN 34305 - 34307.- 2Ad/Jv.- ECF.- Ctra. Km 10 M-512, entre, Navas del Rey y Robledo de Chavela.- 11-05-1973.- Donó: C. A. de Madrid (U.Z.A.).

MNCN 34308.- Ad.- ECF.- Alcalá de Henares.- UTM: VK/6881.- [587]m.02-02-1981.- Donó: C. A. de Madrid (U.Z.A.).

MNCN 34309.- Ad.- ECF.- Alpedrete.- UTM: VL/1301.- [919]m.- 27-051973.- Donó: C. A. de Madrid (U.Z.A.)

MNCN 34310 - 34314.- 5Ad.- ECF.- Alpedrete.- UTM: VL/1301.[919]m.- 03-06-1973.- Donó: C. A. de Madrid (U.Z.A.).

MNCN 34315 - 34317.- 3Ad.- ECF.- Alpedrete.- UTM: VL/1301.[919]m.- 31-05-1973.- Donó: C. A. de Madrid (U.Z.A.).

MNCN 34318 - 34320.- Jv/2Ad.- ECF.- Aranjuez.- UTM: VK/4832.[494]m.- 17-08-1978.- Donó: C. A. de Madrid (U.Z.A.).

MNCN 34321 - 34322.- Ad.- ECF.- Aranjuez.- UTM: VK/4832.- [494]m.08-08-1978.- Donó: C. A. de Madrid (U.Z.A.).

MNCN 34323.- Ad.- ECF.- Laguna de Ontígola, Aranjuez.- UTM: VK/4930.- [560]m.- 12-10-1975.- Donó: Donó: C. A. de Madrid (U.Z.A.).

MNCN 34324 - 34326.- Jv/H/Jv.- ECF.- Becerril de la Sierra.- UTM: VL/1608.- [1073]m.- 11-10-1975.- Donó: Donó: C. A. de Madrid (U.Z.A.).

MNCN 34327 - 34328.- JV.- ECF.- Bustarviejo.- UTM: VL/4023.[1222]m.- 20-04-1969.- Donó: C. A. de Madrid (U.Z.A.)

MNCN 34329.- Jv.- ECF.- Puerto de Canencia, Bustarviejo-Canencia.- UTM: VL/3524.- [1600]m.- 01-04-1973.- Donó: Donó: C. A. de Madrid (U.Z.A.).

MNCN 34330 - 34335.- 6(Ad+Jv).- ECF.- Cercedilla.- UTM: VL/1010.[1188]m.- 04/06-05-1972.- Donó: C. A. de Madrid (U.Z.A.).

MNCN 34336 - 34338.- 3Ad.- ECF.- Cercedilla.- UTM: VL/1010.[1188]m.- 26/28-05-1974.- Donó: C. A. de Madrid (U.Z.A.).

MNCN 34339 - 34347.- 9Ad.- ECF.- Cercedilla.- UTM: VL/1010.[1188]m.- 01-06-1974.- Donó: C. A. de Madrid (U.Z.A.).

MNCN 34348 - 34350.- ECF.- Cercedilla.- UTM: VL/1010.- [1188]m.- 1405-1976.- Donó: C. A. de Madrid (U.Z.A.). 
MNCN 34351 - 34359.- 9Ad.- ECF.- Puerto de La Fuenfría, Cercedilla.UTM: VL/1016.- (1796)m.- 05-08-1979.- C. A. de Madrid (U.Z.A.)

MNCN 34361.- Ad.- ECF.- Collado-Villalba.- UTM: VK/1699.- [917]m.17-07-1971.- Donó: C. A. de Madrid (U.Z.A.).

MNCN 34362.- Ad.- ECF.- Colmenar Viejo.- UTM: VL/3501.- [883]m.01-02-1970.- Donó: C. A. de Madrid (U.Z.A.).

MNCN 34363.- Jv.- ECF.- Colmenar Viejo.- UTM: VL/3501.- [883]m.- 2602-1970.- Donó: C. A. de Madrid (U.Z.A.).

MNCN 34364 - 34365.- Ad/Jv.- ECF.- Colmenar Viejo.- UTM: VL/3501.[883]m.- 01-05-1974.- Donó: C. A. de Madrid (U.Z.A.).

MNCN 34366.- Ad.- ECF.- Soto de Viñuelas, Tres Cantos.- UTM: VK/4195. - [660-700]m.- 18-05-1981 - Donó: C. A. de Madrid (U.Z.A.).

MNCN 34367 - 34381.- Ads.- ECF.- Colmenarejo.- UTM: VK/1390.[872]m.- 07-06-1973.- Donó: C. A. de Madrid (U.Z.A.).

MNCN 34382.- Ad.- ECF.- El Berrueco.- UTM: VL/5326.- [925]m.- 0306-1975.- Donó: C. A. de Madrid (U.Z.A.)

MNCN 34383 - 34384.- Ad.- ECF.- Los Pozuelos-Peñaladrones, Manzanares El real.- UTM: VL/2305.- [920-980]m.- 05-07-1981.Donó: C. A. de Madrid (U.Z.A.)

MNCN 34385 - 34387.- Ad.- ECF.- Mataelpino, El Boalo.- UTM VL/2010.- [1100]m.- 04-05-1972.- Donó: C. A. de Madrid (U.Z.A.).

MNCN 34388 - 34390.- Ad.- ECF.- Matalpino, El Boalo.- UTM: VL/2010.- [1100]m.- 24-08-1975.- Donó: C. A. de Madrid (U.Z.A.).

MNCN 34392.- Ad.- ECF.- Embalse de Valmayor, Galapagar.- UTM VK/18.- [831]m.- 27-05-1979.- Donó: C. A. de Madrid (U.Z.A.).

MNCN 34393 - 34396.- ECF.- Horcajuelo de la Sierra.- UTM: VL/5445.[1145]m.- 22/24-07-1973.- Donó: C. A. de Madrid (U.Z.A.)

MNCN 34397.- ECF.- Horcajuelo de la Sierra.- UTM: VL/5445.[1145]m.- 04-01-1973.- Donó: C. A. de Madrid (U.Z.A.).

MNCN 34398.- ECF.- Horcajuelo de la Sierra.- UTM: VL/5445. [1145]m.- 03-08-1976.- Donó: C. A. de Madrid (U.Z.A.).

MNCN 34399 - 34408.- 10(Ad+Jv).- ECF.- Horcajuelo de la Sierra.- UTM: VL/5445.- [1145]m.- 13/19-07-1976.- Donó: C. A. de Madrid (U.Z.A.)

MNCN 34409 - 34410.- ECF.- Horcajuelo de la Sierra.- UTM: VL/5445.[1145]m.- 04/15-08-1973.- Donó: C. A. de Madrid (U.Z.A.)

MNCN 34411 - 34423.- 13(Ad+Jv).- ECF.- Horcajuelo de la Sierra.- UTM VL/5445.- [1145]m.- <[1991].- Donó: C. A. de Madrid (U.Z.A.).

MNCN 34424.- Ad.- ECF.- Hoyo de Manzanares.- UTM: VK/2397.[1001]m.- 29-05-1979.- Donó: C. A. de Madrid (U.Z.A.).

MNCN 34425.- Ad.- ECF.- Hoyo de Manzanares.- UTM: VK/2397. [1001]m.- 03-04-1973.- Donó: C. A. de Madrid (U.Z.A.).

MNCN 34426 - 34335.- 10Ad.- ECF.- Hoyo de Manzanares.- UTM: VK/2397.- [1001]m.- 05-04-1970.- Donó: C. A. de Madrid (U.Z.A.)

MNCN 34436 - 34437.- 2Ad.- ECF.- Hoyo de Manzanares.- UTM: VK/2397.- [1001]m.- 19-03-1970.- Donó: C. A. de Madrid (U.Z.A.).

MNCN 34438.- Ad.- ECF.- La Cabrera.- UTM: VL/4824.- [1038]m.- 1111-1973.- Donó: C. A. de Madrid (U.Z.A.)

MNCN 34439 - 34440.- Jv/Ad.- ECF.- Zarzaquemada, Leganes.- UTM: VK/3465.- [660]m.- 25-11-1977.- Donó: C. A. de Madrid (U.Z.A.)

MNCN 34441 - 34446.- 5(Ad+Jv).- ECF.- Madrid.- UTM: VK/47. [655]m.- 18/19-04-1974.- Donó: C. A. de Madrid (U.Z.A.).

MNCN 34447.- Ad.- ECF.- Madrid.- UTM: VK/47.- [655]m.- 01-081972.- Donó: C. A. de Madrid (U.Z.A.)

MNCN 34448.- Ad.- ECF.- Madrid.- UTM: VK/47.- [655]m.- 02-021979.- Donó: C. A. de Madrid (U.Z.A.).

MNCN 34449.- Ad.- ECF.- Alameda de Osuna (Barajas), Madrid.- UTM VK/4978.- [640]m.- 14-06-1975.- Donó: C. A. de Madrid (U.Z.A.)

MNCN 34450 - 34456.- 7Ad.- ECF.- Barajas, Madrid.- UTM: VK/5180. [622]m.- 24-10-1975.- Donó: C. A. de Madrid (U.Z.A.)

MNCN 34457.- Ad.- ECF.- Barajas, Madrid.- UTM: VK/5180.- [622]m. 19-08-1975.- Donó: C. A. de Madrid (U.Z.A.).

MNCN 34458 - 34466.- 9Ad.- ECF.- Canal de Isabel II, Madrid.- UTM VK/4077.- [680]m.- 26/28-03-1970.- Donó: C. A. de Madrid (U.Z.A.)

MNCN 34467.- Jv.- ECF.- Casa de Campo, Madrid.- UTM: VK/37.- [600650]m.- 31-10-1979.- Donó: Donó: C. A. de Madrid (U.Z.A.).

MNCN 34468 - 34469.- 2Ad.- ECF.- Ciudad Universitaria, Madrid.- UTM: VK/37.- [600]m.- 18/20-05-1970.- Donó: C. A. de Madrid (U.Z.A.)

MNCN 34470.- Jv.- ECF.- Ciudad Universitaria, Madrid.- UTM: VK/37.[600]m.- 14-10-1977.- Donó: C. A. de Madrid (U.Z.A.).

MNCN 34471.- Jv.- ECF.- Ciudad Universitaria, Madrid.- UTM: VK/37. [600]m.- 22-07-1970.- Donó: C. A. de Madrid (U.Z.A.)

MNCN 34472.- Ad.- ECF.- E.T.S.I. Montes (Ciudad Universitaria), Madrid. UTM: VK/3878.- [620]m.- 04-04-1973.- Donó: C. A. de Madrid (U.Z.A.)
MNCN 34473.- Ad.- ECF.- E.T.S.I. Montes (Ciudad Universitaria), Madrid.- UTM: VK/3878.- [620]m.- 18-05-1981.- Donó: C. A. de Madrid (U.Z.A.).

MNCN 34474 - 34477.- 4Ad.- ECF.- E.T.S.I. Montes (Ciudad Universitaria), Madrid.- UTM: VK/3878.- [620]m.- 15-02-1971.- Donó: C. A. de Madrid (U.Z.A.).

MNCN 34478.- Ad.- ECF.- E.T.S.I. Montes (Ciudad Universitaria), Madrid.- UTM: VK/3878.- [620]m.- 23-03-1972.- Donó: C. A. de Madrid (U.Z.A.)

MNCN 34479 - 34506.- 28(Ad+Jv).- ECF.- E.T.S.I. Montes (Ciudad Universitaria), Madrid.- UTM: VK/3878.- [620]m.- 02/26-02-1970.Donó: C. A. de Madrid (U.Z.A.)

MNCN 34507.- Sad.- ECF.- E.T.S.I. Montes (Ciudad Universitaria), Madrid.- UTM: VK/3878.- [620]m.- 12-02-1972.- Donó: C. A. de Madrid (U.Z.A.).

MNCN 34508.- ECF.- E.T.S.I. Montes (Ciudad Universitaria), Madrid.UTM: VK/3878.- [620]m.- 28-10-1970.- Donó: C. A. de Madrid (U.Z.A.).

MNCN 34509.- ECF.- E.T.S.I. Montes (Ciudad Universitaria), Madrid.UTM: VK/3878.- [620]m.- 19-08-1970.- Donó: C. A. de Madrid (U.Z.A.).

MNCN 34510 - 34511.- 2Ad.- ECF.- E.T.S.I. Montes (Ciudad Universitaria), Madrid.- UTM: VK/3878.- [620]m.- 27/28-03-1971.Donó: C. A. de Madrid (U.Z.A.)

MNCN 34512 - 34513.- 2Ad.- ECF.- E.T.S.I. Montes (Ciudad Universitaria), Madrid.- UTM: VK/3878.- [620]m.- 10/21-10-1971.Donó: C. A. de Madrid (U.Z.A.)

MNCN 34514 - 34526.- (11 AdsJv).- ECF.- E.T.S.I. Montes (Ciudad Universitaria), Madrid.- UTM: VK/3878.- [620]m.- 12/27-03-1970.Donó: C. A. de Madrid (U.Z.A.)

MNCN 34527.- ECF.- E.T.S.I. Montes (Ciudad Universitaria), Madrid.UTM: VK/3878.- [620]m.- 01-04-1970.- Donó: C. A. de Madrid (U.Z.A.)

MNCN 34528 - 34529.- 2Ad.- ECF.- E.T.S.I. Montes (Ciudad Universitaria), Madrid.- UTM: VK/3878.- [620]m.- 09-05-1971.- Donó: C. A. de Madrid (U.Z.A.)

MNCN 34530 - 34531.- 2Jv.- ECF.- El Pardo, Madrid.- UTM: VK/3486.[620-720]m.- 20/24-11-1971.- Donó: C. A. de Madrid (U.Z.A.).

MNCN 34532 - 34535.- 4Ad.- ECF.- El Pardo, Madrid.- UTM: VK/3486.[620-720]m.- 16/30-03-1972.- Donó: C. A. de Madrid (U.Z.A.)

MNCN 34536 - 34543.- 8Ad.- ECF.- El Pardo, Madrid.- UTM: VK/3486.[620-720]m.- 30-09-1972.- Donó: C. A. de Madrid (U.Z.A.).

MNCN 34544 - 34546.- 3Ad.- ECF.- El Pardo, Madrid.- UTM: VK/3486.620-720]m.- 10/23-06-1972.- Donó: C. A. de Madrid (U.Z.A.).

MNCN 34547 - 34550.- 4Ad.- ECF.- El Pardo, Madrid.- UTM: VK/3486.620-720]m.- 03/19-04-1972.- Donó: C. A. de Madrid (U.Z.A.).

MNCN 34551 - 34556.- 6Ad.- ECF.- El Pardo, Madrid.- UTM: VK/3486. [620-720]m.- 07/25-05-1972.- Donó: C. A. de Madrid (U.Z.A.)

MNCN 34557.- Ad.- ECF.- El Pardo, Madrid.- UTM: VK/3486.- [620720]m.- 21-02-1972.- Donó: C. A. de Madrid (U.Z.A.)

MNCN 34558 - 34559.- 3Ad.- ECF.- El Pardo, Madrid.- UTM: VK/3486.[620-720]m.- 15/30-07-1972.- Donó: C. A. de Madrid (U.Z.A.).

MNCN 34560.- Ad.- ECF.- El Pardo, Madrid.- UTM: VK/3486.- [620720]m.- 01-05-1979.- Donó: C. A. de Madrid (U.Z.A.).

MNCN 34561.- Ad.- ECF.- El Pardo, Madrid.- UTM: VK/3486.- [620720]m.- 04-08-1973.- Donó: C. A. de Madrid (U.Z.A.)

MNCN 34562 - 34564.- Ad/2Jv.- ECF.- El Retiro, Madrid.- UTM: VK/4274.- [650]m.- 10/29-07-1973.- Donó: C. A. de Madrid (U.Z.A.).

MNCN 34565.- Ad.- ECF.- Facultad de Ciencias (Ciudad Universitaria), Madrid.- UTM: VK/3878.- [610]m.- 02-09-1971.- Donó: C. A. de Madrid (U.Z.A.).

MNCN 34566 - 34568.- M/H/Jv.- ECF.- Hortaleza, Madrid.- UTM: VK/4580.- [700]m.- 14/26-09-1973.- Donó: C. A. de Madrid (U.Z.A.).

MNCN 34569.- Jv.- ECF.- Hortaleza, Madrid.- UTM: VK/4580.- [700]m.08-10-1973.- Donó: C. A. de Madrid (U.Z.A.).

MNCN 34570.- Ad.- ECF.- Hospital Clínico (Ciudad Universitaria), Madrid.- UTM: VK/3977.- [620]m.- 16-09-1970.- Donó: C. A. de Madrid (U.Z.A.).

MNCN 34571.- Ad.- ECF.- Instituto de Frio Industrial y Experiencia (Cdad. Univer.), Madrid.- UTM: VK/3877.- [620]m.- 19-06-1971.Donó: C. A. de Madrid (U.Z.A.)

MNCN 34572.- Ad.- ECF.- Vallecas, Madrid.- UTM: VK/4770.- [627]m.-03-1970.- Donó: C. A. de Madrid (U.Z.A.). 
MNCN 34573 - 34574.- 2Ad.- ECF.- Manzanares El Real.- UTM: VL/2708.- [908]m.- 22/31-06-1978.- Donó: C. A. de Madrid (U.Z.A.).

MNCN 34575.- Ad.- ECF.- Manzanares El Real.- UTM: VL/2708.[908]m.- 24-06-1973.- Donó: C. A. de Madrid (U.Z.A.).

MNCN 34576.- Ad.- ECF.- Manzanares El Real.- UTM: VL/2708. [908]m.- 31-05-1981.- Donó: C. A. de Madrid (U.Z.A.).

MNCN 34577 - 34579.- 3Ad.- ECF.- ¿Collado Cabrón?, Manzanares El Real.- 17-06-1973.- Donó: C. A. de Madrid (U.Z.A.).

MNCN 34580 - 34581.- 2Jv.- ECF.- La Pedriza, Manzanares El Real.UTM: VL/21.- [1400-1700]m.- 05-10-1970.- C. A. de Madrid (U.Z.A.) MNCN 34582.- Ad.- ECF.- La Pedriza, Manzanares El Real.- UTM: VL/21. [1400-1700]m.- 12-08-1979.- Donó: Donó: C. A. de Madrid (U.Z.A.).

MNCN 34583 - 34584.- 2Ad.- ECF.- Sierra de Guadarrama, Mataelpino. UTM: VL/10.- [1200-2200]m.- 24-08-1975.- Donó: C. A. de Madrid (U.Z.A.).

MNCN 34585 - 34586.- 2Ad.- ECF.- Miraflores de la Sierra.- UTM: VL/3518.- [1150]m.- 25-04-1970.- Donó: C. A. de Madrid (U.Z.A.)

MNCN 34587.- Jv.- ECF.- Montejo de la Sierra.- UTM: VL/5545.[1148]m.- 17-05-1970.- Donó: C. A. de Madrid (U.Z.A.).

MNCN 34588 - 34591.- 4Ad.- ECF.- Montejo de la Sierra.- UTM: VL/5545.- [1148]m.- 21-04-1971.- Donó: C. A. de Madrid (U.Z.A.).

MNCN 34592 - 34593.- Ad/Jv.- ECF.- Montejo de la Sierra.- UTM VL/5545.- [1148]m.- 27-03-1971.- Donó: C. A. de Madrid (U.Z.A.).

MNCN 34594.- Ad.- ECF.- Montejo de la Sierra.- UTM: VL/5545. [1148]m.- 01-10-1971.- Donó: C. A. de Madrid (U.Z.A.).

MNCN 34595.- Ad.- ECF.- El Chaparral, Montejo de la Sierra.- UTM VL/5850.- [1470]m.- 08-07-1977.- Donó: C. A. de Madrid (U.Z.A.)

MNCN 34596.- Ad.- ECF.- Moralzarzal.- UTM: VL/1704.- [979]m.- 18 05-1972.- Donó: C. A. de Madrid (U.Z.A.).

MNCN 34597.- Ad.- ECF.- Móstoles.- UTM: VK/2664.- [661]m.- 17-051970.- Donó: C. A. de Madrid (U.Z.A.)

MNCN 34598.- Jv.- ECF.- Orusco.- UTM: VK/8259.- [649]m.- 24-081979.- Donó: C. A. de Madrid (U.Z.A.).

MNCN 34599 - 34601.- 3Ad.- ECF.- Embalse de El Pontón de la Oliva, Patones.- UTM: VL/6226.- [726]m.- 27-03-1978.- Donó: C. A. de Madrid (U.Z.A.)

MNCN 34602.- Ad.- ECF.- Pedrezuela.- UTM: VL/4910.- [800]m.- 09-041978 - Donó: C. A de Madrid (U.Z.A.).

MNCN 34603 - 34609.- 7( $\mathrm{Ad}+\mathrm{Jv})$.- ECF.- Pinilla del Valle.- UTM: VL/3131.- [1095]m.- 16-04-1979.- Donó: C. A. de Madrid (U.Z.A.).

MNCN 34610 - 34615.- 6(Ad+Jv).- ECF.- Pinilla del Valle.- UTM VL/3131.- [1095]m.- 20-09-1979.- Donó: C. A. de Madrid (U.Z.A.).

MNCN 34616 - 34619.- 4Ad.- ECF.- Rascafría.- UTM: VL/2528. [1163]m.- 15-05-1972.- Donó: C. A. de Madrid (U.Z.A.).

MNCN 34620.- Ad.- ECF.- Rascafría.- UTM: VL/2528.- [1163]m.- 10-051974.- Donó: C. A. de Madrid (U.Z.A.).

MNCN 34621 - 34623.- 3Ad.- ECF.- Rascafría.- UTM: VL/2528.- [1163]m.28-03-1970.- Donó: C. A. de Madrid (U.Z.A.)

MNCN 34624.- Ad.- ECF.- Rascafría.- UTM: VL/2528.- [1163]m.- 25-071975.- Donó: C. A. de Madrid (U.Z.A.).

MNCN 34625.- Ad.- ECF.- [Monasterio de] El Paular, Rascafría.- UTM VL/2527.- [1159]m.- 14-06-1970.- Donó: C. A. de Madrid (U.Z.A.).

MNCN 34626.- Ad.- ECF.- Peñalara al Puerto de «Los Cotos», Rascafría.UTM: VL/12.- [1800-2400]m.- 14-06-1970.- Donó: C. A. de Madrid (U.Z.A.).

MNCN 34627.- Ad.- ECF.- Río Lozoya cruce con el puente de Taboada, Gargantilla del Lozoya.- UTM: VL/3032.- [1000]m.- 16-06-1975.- Donó: C. A. de Madrid (U.Z.A.)

MNCN 34628.- Ad.- ECF.- Pantano de San Juan, San Martín de Valdeiglesias.- UTM: UK/87.- [580]m.- 28-03-1975.- Donó: C. A. de Madrid (U.Z.A.)

MNCN 34629 - 34630.- 2Ad.- ECF.- Pantano de San Juan, San Martín de Valdeiglesias.- UTM: UK/87.- [580]m.- verano-1975.- Donó: C. A. de Madrid (U.Z.A.)

MNCN 34631.- Ad.- ECF.- Puerto de Somosierra, Somosierra.- UTM: VL/5154.- [1404]m.- 02-09-1976.- Donó: C. A. de Madrid (U.Z.A.).

MNCN 34632.- Ad.- ECF.- Torrelodones.- UTM: VK/2192.- [845]m.- 18 07-1972.- Donó: C. A. de Madrid (U.Z.A.).

MNCN 34633 - 34641.- Ad/6Jv.- ECF.- Embalse de los Peñascales, Torrelodones.- UTM: VK/2392.- [740-760]m.- 13-09-1973.- Donó: C. A. de Madrid (U.Z.A.)

MNCN 34642 - 34643.- 2Ad.- ECF.- Embalse de los Peñascales, Torrelodones.- UTM: VK/2392.- [740-760]m.- 11/12-05-1974.- Donó C. A. de Madrid (U.Z.A.)
MNCN 34644 - 34646.- 2Ad/Jv.- ECF.- Embalse de los Peñascales, Torrelodones.- UTM: VK/2392.- [740-760]m.- 28-09-1974.- Donó: C. A. de Madrid (U.Z.A.).

MNCN 34647 - 34656.- 10(Ad+Jv).- ECF.- Embalse de los Peñascales, Torrelodones.- UTM: VK/2392.- [740-760]m.- 11-09-1973.- Donó: C. A. de Madrid (U.Z.A.)

MNCN 34657 - 34682.- 27(Ad+Jv).- ECF.- Embalse de los Peñascales, Torrelodones.- UTM: VK/2392.- [740-760]m.- 14-09-1973.- Donó: C. A. de Madrid (U.Z.A.).

MNCN 34683 - 34684.- Ad/Jv.- ECF.- Embalse de los Peñascales, Torrelodones.- UTM: VK/2392.- [740-760]m.- 07-09-1973.- Donó: C. A. de Madrid (U.Z.A.).

MNCN 34685 - 34686.- 2Jv.- ECF.- Embalse de los Peñascales, Torrelodones.- UTM: VK/2392.- [740-760]m.- 12-09-1973.- Donó: C. A. de Madrid (U.Z.A.).

MNCN 34687 - 34688.- Ad/Jv- ECF. Embalse de los Peñascales, Torrelodones.- UTM: VK/2392.- [740-760]m.- 06-09-1973.- Donó: C. A. de Madrid (U.Z.A.).

MNCN 34689 - 34696.- 8Jv.- ECF.- Embalse de los Peñascales, Torrelodones.- UTM: VK/2392.- [740-760]m.- 27-09-1975.- Donó: C. A. de Madrid (U.Z.A.)

MNCN 34697.- Ad.- ECF.- Embalse de los Peñascales, Torrelodones.- UTM: VK/2392.- [740-760]m.- 16-07-1972.- Donó: C. A. de Madrid (U.Z.A.).

MNCN 34698 - 34699.- Ad/Jv.- ECF.- Embalse de los Peñascales, Torrelodones.- UTM: VK/2392.- [740-760]m.- 23-09-1973.- Donó: C. A. de Madrid (U.Z.A.).

MNCN 34702 - 34703.- 2Ad.- ECF.- Embalse de los Peñascales, Torrelodones.- UTM: VK/2392.- [740-760]m.- 17/19-06-1975.- Donó: C. A. de Madrid (U.Z.A.).

MNCN 34704 - 34707.- 4Ad.- ECF.- Embalse de los Peñascales, Torrelodones.- UTM: VK/2392.- [740-760]m.- 06-09-1976.- Donó: C. A. de Madrid (U.Z.A.).

MNCN 34708 - 34713.- 2Ad/4Jv.- ECF.- Embalse de los Peñascales, Torrelodones.- UTM: VK/2392.- [740-760]m.- 02-09-1973.- Donó: C. A. de Madrid (U.Z.A.)

MNCN 34714 - 34716.- 3Ad.- ECF.- Valdemoro.- UTM: VK/4249.[615]m.- 16-04-1973.- Donó: C. A. de Madrid (U.Z.A.).

MNCN 34717.- Jv.- ECF.- Venturada.- UTM: VL/4716.- [864]m.- 31-031978.- Donó: C. A. de Madrid (U.Z.A.).

MNCN 34718 - 34720.- ECF.- Villanueva de la Cañada.- UTM: VK/1578.[652]m.- 06-07-1973.- Donó: C. A. de Madrid (U.Z.A.).

MNCN 34721 - 34722.- 2Ad.- ECF.- Villanueva de la Cañada.- UTM: VK/1578.- [652]m.- 07-03-1971.- Donó: C. A. de Madrid (U.Z.A.).

MNCN 34723.- ECF.- Villanueva de la Cañada.- UTM: VK/1578.[652]m.- 07-05-1973 - Donó: C. A. de Madrid (U.Z.A.).

MNCN 34724.- ECF.- Villanueva de la Cañada.- UTM: VK/1578.[652]m.- 19-03-1973.- Donó: C. A. de Madrid (U.Z.A.).

MNCN 34725 - 34726.- ECF.- Villanueva de la Cañada.- UTM: VK/1578.[652]m.- 01-11-1973.- Donó: C. A. de Madrid (U.Z.A.)

MNCN 34727 - 34728.- 2Ad.- ECF.- Villanueva de la Cañada.- UTM: VK/1578.- [652]m.- 31-03-1974.- Donó: C. A. de Madrid (U.Z.A.).

MNCN 34729.- Ad.- ECF.- El Pardo, Madrid.- UTM: VK/3486.- [620720]m.- 06-04-1978.- Donó: C. A. de Madrid (U.Z.A.).

MNCN 35821.- H.- Sad.- ECF.- Hoyo de Manzanares.- UTM: VK/2397.[1001]m.- 05-04-1970.- Donó: C. A. de Madrid (U.Z.A.).

MNCN 36255.- Jv.- ECF.- Madrid.- UTM: VK/47.- [655]m.- 26-03-1978.Donó: C. A. de Madrid (U.Z.A.).

MNCN 36256 - 36257.- Ad/Jv.- ECF.- Madrid.- UTM: VK/47.- [655]m.19-04-1974.- Donó: C. A. de Madrid (U.Z.A.).

MNCN 36258.- Jv.- ECF.- Ciudad Universitaria, Madrid.- UTM: VK/37.[600]m.- 15-05-1972.- Donó: C. A. de Madrid (U.Z.A.).

MNCN 36259 - 36260.- 2Ad/Sad.- ECF.- El Pardo, Madrid.- UTM: VK/3486.- [620-720]m.- 21-02-1972.- C. A. de Madrid (U.Z.A.).

MNCN 36261.- Ad.- ECF.- El Pardo, Madrid.- UTM: VK/3486.- [620720]m.- 13-04-1972.- Donó: C. A. de Madrid (U.Z.A.).

MNCN 36262 - 36263.- Ad/Sad.- ECF.- El Pardo, Madrid.- UTM: VK/3486.- [620-720]m.- 18/25-05-1972.- C. A. de Madrid (U.Z.A.).

MNCN 36264 - 36265.- 2Sad.- ECF.- El Pardo, Madrid.- UTM: VK/3486.[620-720]m.- 10/23-06-1972.- Donó: C. A. de Madrid (U.Z.A.).

MNCN 36266 - 36268.- Ad/Sad.- ECF.- El Pardo, Madrid.- UTM: VK/3486.- [620-720]m.- 30-09-1972.- Donó: C. A. de Madrid (U.Z.A.).

MNCN 38697 - 38699.- 2Ad/JV.- ECF.- Embalse de los Peñascales, Torrelodones.- UTM: VK/2392.- [740-760]m.- 15-04-1976.- Donó: C. A. de Madrid (U.Z.A.) 
MNCN 39510 - 39512.- Ad/2Jv.- ECF.- El Escorial.- UTM: VK/0493.[909]m.- 01/07-06-1972.- Donó: C. A. de Madrid (U.Z.A.)

MNCN 39756.- Ad.- ECF.- Madrid.- UTM: VK/47.- [655]m.- 03-08 1982.- Donó: C. A. de Madrid (U.Z.A.).

MNCN 41511 - 41518.- 8(Ad+Jv).- ECF.- Pelayos de la Presa.- UTM: UK/8568.- [570]m.- <[20-10-2000].- Colectó: C. Olmos.

MNCN 41707 - 41708.- 2Ad.- ECF.- Presa del Pantano de Valmayor, Valdemorillo.- UTM: VK/3087.- [800]m.- 18-03-2000.- Colectó y donó: J. E. González.

Podarcis muralis (Laurenti, 1768)

MNCN 6373.- Jv.- ECF.- Puerto de La Fuenfría, Cercedilla.- UTM VL/1016.- 1400 -1600m.- 17-06-1980.- Colectó y donó: J. Mellado.

MNCN 6521 - 6527.- 7Ad.- ECF.- Puerto de La Fuenfría, Cercedilla.UTM: VL/1016.- (1796)m.- 04-07-1980.- Colectó y donó: J. Mellado.

MNCN 6528 - 6531.- 4Ad.- ECF.- Puerto de La Fuenfría, Cercedilla. UTM: VL/1016.- (1796)m.- 09-07-1980.- Colectó y donó: J. Mellado.

MNCN 6532 - 6537.- 3Ad/Jv/3Ad.- ECF.- Cercedilla.- UTM: VL/1010.[1188]m.- 17-06-1980.- Colectó y donó: J. Mellado.

MNCN 6544 - 6547.- Ad.- ECF.- Puerto de «Los Cotos», Rascafría.- UTM: VL/1919.- [1830]m.- 26-06-1980.- Colectó y donó: J. Mellado.

MNCN 6548 - 6549.- Sad/Ad.- ECF.- Umbría de la Fuenfría, Cercedilla. UTM: VL/11.- [1700-1800]m.- 28-06-1980.- Colectó y donó: J. Mellado.

MNCN 6550 - 6554.- JV/4Ad.- ECF.- Cercedilla.- UTM: VL/1010.[1188]m.- 29-05-1980.- Colectó y donó: J. Mellado.

MNCN 6599 - 6602.- 2Ad/Jv/Ad.- ECF.- Navacerrada.- UTM: VL/1409. [1203]m.- 20-05-1980.- Colectó y donó: J. Mellado.

MNCN 6654 - 6655.- 2Ad.- ECF.- Río Lozoya, Rascafría.- UTM: VL/22.1500m.- 24-05-1970.- Colectó y donó: [J. M. Rey \& A. Landín].

MNCN 6767 - 6769.- 3Ad.- ECF.- Barranco de Cienvallejos ${ }^{(*)}$, Brunete.UTM: VK/1769.- 25-09-1927.- Colectó y donó: C. Bolívar.

OBSERVACIONES: ${ }^{(*)}$ Como ya se indicó anteriormente, representa una cita totalmente fuera del área de distribución de la especie. Pese a tener $\mathrm{n}^{\circ} \mathrm{de}$ entrada 1927.09.29.01-03, no está recogidos en el «Libro de registro de entradas de ejemplares en la colección de Zoología, sección de Osteozoología, 1911-1932» p. 200, por no existir anotaciones, en dicho libro, entre el 23 de junio y el 30 de septiembre (AMNCN, 1911).

MNCN 6911.- Jv.- ECF.- El Ventorrillo, Mancomunidad de Cercedilla y Navacerrada.- UTM: VL/1312.- [1500]m.- <[01-04-1931].- Colectó y donó: E. Morales-Agacino.

OBSERVACIONES: Pese a tener $\mathrm{n}^{0}$ de entrada 1931.04.01.01-02, no esta Recogido en el «Libro de registro de entradas de ejemplares en la colección de Zoología, sección de Osteozoología, 1911-1932» pp 200, por no existir anotaciones, en dicho libro, entre el 7 de marzo y el 10 de abril, (AMNCN, 1911).

MNCN 6944.- Ad.- ECF.- El Escorial.- UTM: VK/0493.- [909]m.-- -04/051880.- Colectó y donó: E. Boscá.

MNCN 7103.- Ad.- ECF.- El Escorial.- UTM: VK/0493.- [909]m.Colectó: A. Zulueta.

MNCN 9362 - 9363.- 2Jv.- ECF.- Puerto de «Los Cotos», Rascafría. UTM: VL/1919.- [1830]m.- 11-09-1983.- Colectó y donó: J. Dorda \& M. Ga-París.

MNCN 23255.- Ad.- EDC.- La Fuenfría, Cercedilla.- UTM: VL/1016.[1793]m.- 04-07-1980.- Colectó y donó: J. Mellado.

MNCN 35525.- Ad.- ECF.- Montejo de la Sierra.- UTM: VL/5545. [1148]m.- 28-10-1969.- Donó: C. A. de Madrid (U.Z.A.).

MNCN 36218.- Ad.- ECF.- Puerto de Canencia, Bustarviejo-Canencia.UTM: VL/3524.- [1600]m.- 20-12-1971.- Donó: C. A. de Madrid (U.Z.A.)

MNCN 36219 - 36223.- 3Ad/2Jv.- ECF.- Cercedilla.- UTM: VL/1010.[1188]m.- 29/31-05-1974.- Donó: C. A. de Madrid (U.Z.A.)

MNCN 36224 - 36226.- 3Ad.- ECF.- Cercedilla.- UTM: VL/1010.[1188]m.- 15-05-1973.- Donó: C. A. de Madrid (U.Z.A.).

MNCN 36227 - 36232.- 6Ad.- ECF.- Cercedilla.- UTM: VL/1010.[1188]m.- 02/06-05-1972.- Donó: C. A. de Madrid (U.Z.A.)

MNCN 36233.- Ad.- ECF.- Cercedilla.- UTM: VL/1010.- [1188]m.- 28/3005-1978.- Donó: C. A. de Madrid (U.Z.A.).

MNCN 36234 - 36235.- 2Ad.- ECF.- Collado de las Marichivas, Cercedilla.- UTM: VL/0815.- [1700-1800]m.- 28/30-05-1973.- Donó: C. A. de Madrid (U.Z.A.).

MNCN 36236 - 36237.- 2Ad.- ECF.- Debajo de Fuenfría, Cercedilla.- UTM: VL/1016.- [1700-1800]m.- 31-05-1973.- Donó: C. A. de Madrid (U.Z.A.)

MNCN 36238 - 36239.- 2Ad.- ECF.- Por debajo del Puerto de la Fuenfría, Cercedilla.- UTM: VL/1016.- [1700-1800]m.- 01-06-1973.- Donó: C. A de Madrid (U.Z.A.).
MNCN 36240 - 36241.- Ad/Jv.- ECF.- Debajo del Collado de Marichivas, Cercedilla.- UTM: VL/0914.- [1700-1800]m.- 29/30-05-1973.- Donó: C. A. de Madrid (U.Z.A.)

MNCN 36242 - 36243.- 2Ad.- ECF.- Entre la Fuenfría y el Collado de las ¿Chivas? ${ }^{(*)}$, Cercedilla.- UTM: VL/00.- [1800]m.- 27-05-1973.- Donó: C. A. de Madrid (U.Z.A.). OBSERVACIONES: $(*)$ Quizás quisieron referirse a Marichivas.

MNCN 36244 - 36247.- 4Ad.- ECF.- Entre la Fuenfría y el Collado de las ¿Chivas?(*), Cercedilla.- UTM: VL/00.- [1800]m.- 27-05-1973.- Donó: C. A. de Madrid (U.Z.A.).

OBSERVACIONES: $(*)$ Quizás quisieron referirse a Marichivas.

MNCN 36248 - 36250.- 2Jv/Ad.- ECF.- Las Dehesas, Cercedilla.- UTM: VL/1013.- [1400-1600]m.- 26/31-05-1973.- C. A. de Madrid (U.Z.A.).

MNCN 36251.- Ad.- ECF.- Puerto de Navafría [=Puerto de Lozoya] (vertiente Sur), Lozoya.- UTM: VL/3137.- (1760)m.- 05-06-1979.- Donó: C. A. de Madrid (U.Z.A.)

MNCN 36269 - 36270.- 2Ad.- ECF.- Montejo de la Sierra.- UTM: VL/5545.- [1148]m.- 20/14-07-1972.- Donó: C. A. de Madrid (U.Z.A.).

MNCN 36271.- Ad.- ECF.- El Chaparral, Montejo de la Sierra.- UTM: VL/5850.- [1470]m.- 06-05-1973.- C. A. de Madrid (U.Z.A.).

MNCN 36272 - 36273.- 3Ad.- ECF.- El Chaparral, Montejo de la Sierra.UTM: VL/5850.- [1470]m.- 25-03-1975.- C. A. de Madrid (U.Z.A.).

MNCN 36275.- Ad.- ECF.- Montejo de la Sierra.- UTM: VL/5545.[1148]m.- 22-08-1971.- Donó: C. A. de Madrid (U.Z.A.).

MNCN 36276 - 36278.- 3Ad.- ECF.- Rascafría.- UTM: VL/2528. [1163]m.- 02-05-1970.- Donó: C. A. de Madrid (U.Z.A.)

MNCN 36279 - 36286.- 8Ad.- ECF.- Puerto de Los Cotos (=Puerto del Paular), Rascafría.- UTM: VL/1919.- (1830)m.- 01/31-05-1970.- Donó: C. A. de Madrid (U.Z.A.).

MNCN 36287.- Ad.- ECF.- Peñalara al Puerto de «Los Cotos», Rascafría.UTM: VL/12.- [1800-2400]m.- 03-05-1970.- Donó: C. A. de Madrid (U.Z.A.).

MNCN 36288.- Ad.- ECF.- Laguna de Peñalara, Rascafría.- UTM: VL/1921.- [1990]m.- 13-07-1976.- Donó: C. A. de Madrid (U.Z.A.)

MNCN 36289 - 36290.- Ad.- ECF.- Laguna de Peñalara, Rascafría.- UTM: VL/1921.- [1990]m.- 20-09-1975.- C. A. de Madrid (U.Z.A.)

MNCN 36291.- Ad.- ECF.- Nacimiento del Lozoya [io de la Angostura o de Peñalara?], Rascafría.- 13-08-1969.- Donó: C. A. de Madrid (U.Z.A.).

MNCN 36292.- Jv.- ECF.- La Pedriza, Manzanares El Real.- UTM: VL/21.- [1400-1700]m.- 05-10-1970.- Donó: C. A. de Madrid (U.Z.A.).

Psammodromus algirus Linnaeus, 1758

MNCN 6362.- Ad.- ECF.- Alpedrete.- UTM: VL/1301.- [919]m.- 25-061980.- Colectó y donó: J. Mellado.

MNCN 6363.- Ad.- ECF.- Navacerrada.- UTM: VL/1409.- [1203]m.- 0107-1980.- Colectó y donó: J. Mellado.

MNCN 6365 - 6367.- 3Ad.- ECF.- Navacerrada.- UTM: VL/1409.[1203]m.- 23-05-1980.- Colectó y donó: J. Mellado.

MNCN 6415 - 6416.- 2Ad.- ECF.- Junto al camino de la casa de «E Ventorrillo», Mancomunidad de Cercedilla-Navacerrada.- UTM: 30TVL/1312.- [1500]m.- 04-08-1980.

MNCN 7664.- Ad.- ECF.- Madrid.- UTM: VK/47.- [655]m.- -07-1905.Colectó y donó: L. Lozano Rey.

MNCN 7786 - 7787.- Ad.- ECF.- San Lorenzo de El Escorial.- UTM: VK/0393.- [1032]m.- -07-1892.- Colectó y donó: E. Boscá. OBSERVACIONES: Recogidos en el «Libro de registro de entradas de ejemplares en la colección de Zoología, sección de Osteozoología, 19111932» pp 68, (AMNCN, 1911).

MNCN 7803 - 7804.- Ad/Jv.- ECF.- San Lorenzo de El Escorial.- UTM: VK/0393.- [1032]m.

MNCN 9196 - 9197.- Ad.- ECF.- Aranjuez.- UTM: VK/4832.- [494]m.17-05-1975.- Colectó y donó: I. Doadrio.

MNCN 15996.- Ad/?.- EDP.- Cercedilla.- UTM: VL/1010.- 1.200m.18/19-06-1982.- Colectó y donó: J. Delibes \& A. Larramendi.

MNCN 15997.- Ad/?.- EDP.- Cercedilla.- UTM: VL/1010.- 1.200m.18/19-06-1982.- Colectó y donó: J. Delibes \& A. Larramendi.

MNCN 15998.- $\mathrm{Ad} /$ ?.- EDP.- Cercedilla.- UTM: VL/1010.- 1.200m.18/19-06-1982.- Colectó y donó: J. Delibes \& A. Larramendi.

MNCN 15999.- Ad/?.- EDP.- Cercedilla.- UTM: VL/1010.- 1.200m.18/19-06-1982.- Colectó y donó: J. Delibes \& A.Larramendi.

MNCN 16000.- Ad.- EDC.- Arroyo Dehesilla (La Pedriza), Manzanares El Real.- UTM: VL/2513.- [1200]m.- 03-08-1982.- Colectó y donó: M. García París. 
MNCN 36678.- Ad.- ECF.- Pelayos de la Presa.- UTM: UK/8568.[570]m.- 29-05-1977.- Donó: C. A. de Madrid (U.Z.A.)

MNCN 36739 - 36746.- 6Ad/2Jv.- ECF.- Villanueva de la Cañada.- UTM: VK/1578.- [652]m.- 23-06-1974.- Donó: C. A. de Madrid (U.Z.A.).

MNCN 36798.- Ad.- ECF.- Villanueva de la Cañada.- UTM: VK/1578. [652]m.- 19-03-1973.- Donó: C. A. de Madrid (U.Z.A.).

MNCN 36806 - 36820.- $13 \mathrm{Ad} / 2 \mathrm{jv}$.- ECF.- Villanueva de la Cañada.- UTM VK/1578.- [652]m.- 06/19-05-1973.- Donó: C. A. de Madrid (U.Z.A.).

MNCN 36829 - 36830.- Ad/Jv.- ECF.- Villanueva de la Cañada.- UTM VK/1578.- [652]m.- 09-05-1971.- Donó: C. A. de Madrid (U.Z.A.)

MNCN 36831.- Ad.- ECF.- Villanueva de la Cañada.- UTM: VK/1578.[652]m.- 16-04-1972.- Donó: C. A. de Madrid (U.Z.A.).

MNCN 36992.- Ad.- ECF.- M-126 (=Ctra. de Buitrago de Lozoya a, Manjirón (=Puentes Viejas).- UTM: VL/43.- [970-1070]m.- 24-051973.- Donó: C. A. de Madrid (U.Z.A.).

MNCN 36994 - 36996.- 3Ad.- ECF.- Alpedrete.- UTM: VL/1301.[919]m.- 31-05-1973.- Donó: C. A. de Madrid (U.Z.A.).

MNCN 36997.- Ad.- ECF.- Alpedrete.- UTM: VL/1301.- [919]m.- 03-061973.- Donó: C. A. de Madrid (U.Z.A.).

MNCN 36998.- Ad.- ECF.- Aranjuez.- UTM: VK/4832.- [494]m.- 21-051973.- Donó: C. A. de Madrid (U.Z.A.).

MNCN 36999.- Ad.- ECF.- ¿Dehesa de?, Arganda del Rey.- UTM: VK66.[650-700]m.- 24-03-1973.- Donó: C. A. de Madrid (U.Z.A.).

MNCN 37000.- Ad.- ECF.- Puerto de Canencia, Bustarviejo-Canencia.UTM: VL/3524.- [1600]m.- 01-04-1973.- Donó: C. A. de Madrid (U.Z.A.)

MNCN 37001.- Ad.- ECF.- Puerto de Canencia, Bustarviejo-Canencia.UTM: VL/3524.- [1600]m.- 03-06-1973.- Donó: C. A. de Madrid (U.Z.A.)

MNCN 37002.- Ad.- ECF.- Puerto de Canencia, Bustarviejo-Canencia. UTM: VL/3524.- [1600]m.- 27-05-1973.- Donó: C. A. de Madrid (U.Z.A.)

MNCN 37003.- Ad.- ECF.- Cerceda, El Boalo.- UTM: VL/2105.- [949]m.05-07-1981.- Donó: C. A. de Madrid (U.Z.A.).

MNCN 37004 - 37005.- 2Ad.- ECF.- Cercedilla.- UTM: VL/1010.[1188]m.- 14-05-1976.- Donó: C. A. de Madrid (U.Z.A.).

MNCN 37006 - 37007.- 2Ad.- ECF.- Chapineria.- UTM: UK/9770. [680]m.- 31-05-1971.- Donó: C. A. de Madrid (U.Z.A.)

MNCN 37008.- JV.- ECF.- Chinchón.- UTM: VK/6443.- [753]m.- 08-051975.- Donó: C. A. de Madrid (U.Z.A.)

MNCN 37009 - 37013.- 4Ad/Jv.- ECF.- Collado-Villalba.- UTM VK/1699.- [917]m.- 17-07-1971.- Donó: C. A. de Madrid (U.Z.A.)

MNCN 37014.- Ad.- ECF.- Colmenarejo.- UTM: VK/1390.- [872]m.- 0706-1973.- Donó: C. A. de Madrid (U.Z.A.).

MNCN 37015.- Ad.- ECF.- Mataelpino, El Boalo.- UTM: VL/2010. [1100]m - 04-05-1972 - Donó: C. A. de Madrid (U.Z.A.)

MNCN 37016 - 37017.- 2Ad.- ECF.- La Herrería, El Escorial.- UTM VK/0293.- [880]m.- 15-05-1979.- Donó: C. A. de Madrid (U.Z.A.).

MNCN 37018.- Ad.- ECF.- El Escorial.- UTM: VK/0493.- [909]m.- 01-04 1972.- Donó: C. A. de Madrid (U.Z.A.).

MNCN 37019.- Jv.- ECF.- El Escorial.- UTM: VK/0493.- [909]m.- 26-061972.- Donó: C. A. de Madrid (U.Z.A.)

MNCN 37020.- Jv.- ECF.- Horcajuelo de la Sierra.- UTM: VL/5445.[1145]m.- 11-07-1976.- Donó: C. A. de Madrid (U.Z.A.).

MNCN 37021.- Ad.- ECF.- Hoyo de Manzanares.- UTM: VK/2397.[1001]m.- 04-03-1970.- Donó: C. A. de Madrid (U.Z.A.)

MNCN 37022 - 37024.- 3Ad.- ECF.- Hoyo de Manzanares.- UTM VK/2397.- [1001]m.- 05/08-04-1980.- Donó: C. A. de Madrid (U.Z.A.)

MNCN 37025.- Jv.- ECF.- Hoyo de Manzanares.- UTM: VK/2397. [1001]m.- 29-05-1974.- Donó: C. A. de Madrid (U.Z.A.).

MNCN 37026 - 37035.- Ad/Sad/Jv.- ECF.- Casa de Campo, Madrid. UTM: VK/37.- [600-650]m.- 26-06-1974.- C. A. de Madrid (U.Z.A.)

MNCN 37036 - 37038.- 3Ad.- ECF.- Embalse de los Peñascales, Torrelodones.- UTM: VK/2392.- [740-760]m.- 15/24-06-1973.- Donó: C. A. de Madrid (U.Z.A.)

MNCN 37039.- Ad.- ECF.- E.T.S.I. Montes (Ciudad Universitaria), Madrid.- UTM: VK/3878.- [620]m.- 17-06-1970.- Donó: C. A. de Madrid (U.Z.A.)

MNCN 37041 - 37058.- 18 (Ad+Jv).- ECF.- El Pardo, Madrid.- UTM VK/3486.- [620-720]m.- 07/10-09-1971.- Donó: C. A. de Madrid (U.Z.A.)

MNCN 37059 - 37061.- 3Ad.- ECF.- El Pardo, Madrid.- UTM: VK/3486. [620-720]m - 16-04-1972 - Donó: C. A de Madrid (U.Z.A.)

MNCN 37062 - 37078.- 17(Ad+Jv).- ECF.- El Pardo, Madrid.- UTM VK/3486.- [620-720]m.- 05/25-05-1972.- Donó: C.A.de Madrid (U.Z.A.)
MNCN 37079 - 37082.- 3Ad/Jv.- ECF.- El Pardo, Madrid.- UTM: VK/3486- [620-720]m. 10/23-06-1972 - C. A. de Madrid (U.Z.A.).

MNCN 37083 - 37091.- 9(Ad+Jv).- ECF.- El Pardo, Madrid.- UTM: VK/3486.- [620-720]m.- 03/30-09-1972.- Donó: C. A. de Madrid (U.Z.A.).

MNCN 37092.- Ad.- ECF.- El Pardo, Madrid.- UTM: VK/3486.- [620720]m.- 21-04-1969.- Donó: C. A. de Madrid (U.Z.A.)

MNCN 37093 - 37094.- Sad/Ad.- ECF.- El Pardo, Madrid.- UTM: VK/3486- [620-720]m.- 05-08-1973 - Donó: C. A. de Madrid (U.Z.A.).

MNCN 37095.- Jv.- ECF.- Mingorrubio, Madrid.- UTM: VK/3387.[600]m.- 03-05-1981.- Donó: C. A. de Madrid (U.Z.A.).

MNCN 37096.- Ad.- ECF.- Sierra de Guadarrama, Mataelpino.- UTM: VL/10.- [1200-2200]m.- 24-08-1975.- Donó: C. A. de Madrid (U.Z.A.). MNCN 37097.- Ad.- ECF.- Montejo de la Sierra.- UTM: VL/5545.[1148]m.- 20-07-1972.- Donó: C. A. de Madrid (U.Z.A.)

MNCN 37098 - 37100.- 3Ad.- ECF.- Moralzarzal.- UTM: VL/1704.[979]m.- 12-05-1972.- Donó: C. A. de Madrid (U.Z.A.)

MNCN 37101.- Ad.- ECF.- Robledo de Chavela.- UTM: UK/9584.[903]m.- 02-06-1974.- Donó: C. A. de Madrid (U.Z.A.).

MNCN 37102.- 1Jv.- ECF.- Robledo de Chavela.- UTM: UK/9584.[903]m.- 01-06-1984.- Donó: C. A. de Madrid (U.Z.A.)

MNCN 37103.- JV.- ECF.- ¿Cueva de los Osos? (Pantano de San Juan), San Martín de Valdeiglesias.- UTM: UK/87.- 27-03-1975.- Donó: C. A. de Madrid (U.Z.A.).

MNCN 37104 - 37106.- 3Ad.- ECF.- Pantano de San Juan, San Martín de Valdeiglesias.- UTM: UK/87.- [580]m.- 11-05-1975.- Donó: C. A. de Madrid (U.Z.A.)

MNCN 37107.- Sad.- ECF.- Soto del Real.- UTM: VL/3411.- [921]m.- 2305-1974.- Donó: C. A. de Madrid (U.Z.A.).

MNCN 37108.- Sad.- ECF.- Torrelodones.- UTM: VK/2192.- [845]m.$<$ [1991].- Donó: C. A. de Madrid (U.Z.A.)

MNCN 37109.- Jv.- ECF.- E.T.S.I. Montes (Ciudad Universitaria), Madrid.- UTM: VK/3878.- [620]m.- 20-03-1970.- Donó: C. A. de Madrid (U.Z.A.)

MNCN 37110.- Ad.- ECF.- Embalse de los Peñascales, Torrelodones.UTM: VK/2392.- [740-760]m.- 13-03-1974.- Donó: C. A. de Madrid (U.Z.A.).

MNCN 37111.- Ad.- ECF.- Embalse de los Peñascales, Torrelodones.UTM: VK/2392.- [740-760]m.- 11-05-1974.- Donó: C. A. de Madrid (U.Z.A.).

MNCN 37112 - 37123.- $11 \mathrm{Ad} / \mathrm{JV}$.- ECF.- Embalse de los Peñascales, Torrelodones.- UTM: VK/2392.- [740-760]m.- 17/19-06-1975.- Donó: C. A. de Madrid (U.Z.A.)

MNCN 37124.- Jv.- ECF.- Embalse de los Peñascales, Torrelodones.UTM: VK/2392.- [740-760]m.- 27-09-1975.- Donó: C. A. de Madrid (U.Z.A.).

MNCN 37125 - 37128.- 2Ad/2Jv.- ECF.- Embalse de los Peñascales, Torrelodones.- UTM: VK/2392.- [740-760]m.- 15-04-1976.- Donó: C. A. de Madrid (U.Z.A.).

MNCN 37129.- Ad.- ECF.- Embalse de los Peñascales, Torrelodones.UTM: VK/2392.- [740-760]m.- 15-05-1976.- Donó: C. A. de Madrid (U.Z.A.).

MNCN 37143 - 37146.- 4Ad - ECF - El Pardo, Madrid - UTM: VK/3486. [620-720]m.- 13/18-07-1972.- Donó: C. A. de Madrid (U.Z.A.).

MNCN 37302.- Ad.- ECF.- E.T.S.I. Montes (Ciudad Universitaria), Madrid.- UTM: VK/3878.- [620]m.- 04-04-1970.- Donó: C. A. de Madrid (U.Z.A.).

MNCN 37303.- Ad.- ECF.- E.T.S.I. Montes (Ciudad Universitaria), Madrid.- UTM: VK/3878.- [620]m.- 26-05-1970.- Donó: C. A. de Madrid (U.Z.A.)

MNCN 37304.- Ad.- ECF.- Soto de Viñuelas, Tres Cantos.- UTM: VK/4195.- [660-700]m.- 25-04-1981.- Donó: C. A. de Madrid (U.Z.A.).

MNCN 40067.- Ad.- ECF.- Villanueva de la Cañada.- UTM: VK/1578.[652]m.- 16-04-1972.- Donó: C. A. de Madrid (U.Z.A.).

MNCN 40071.- Jv.- ECF.- Collado-Villalba.- UTM: VK/1699.- [917]m.$<$ [1991].- Colectó: S. Savia.- Donó: C. A. de Madrid (U.Z.A.).

MNCN 40076.- Ad.- ECF.- Alcalá de Henares.- UTM: VK/6881.- [587]m.11-10-1983.- Donó: C. A. de Madrid (U.Z.A.)

MNCN 40077.- Ad.- ECF.- Navas del Rey.- UTM: UK/9371.- [709]m.- 1205-1985.- Donó: C. A. de Madrid (U.Z.A.)

MNCN 40088 - 40089.- 2Jv.- ECF.- Bosque de la Herrería, San Lorenzo de El Escorial.- UTM: VK/0293.- [1000]m.-- -10-1983.- Donó: C. A. de Madrid (U.Z.A.). 
MNCN 40132.- 8Hu.- ECF.- El Escorial.- UTM: VK/0493.- [909]m.- 2206-1968.- Donó: C. A. de Madrid (U.Z.A.).

MNCN 40806.- Pu.- ECF.- Manjirón (=Puentes viejas).- UTM 39TVL/5135.- [1012]m.- 23-08-2001.- Colectó y donó: Ma Valladolid.

MNCN 40807.- Pm.- ETC, Manjirón (=Puentes viejas).- UTM: 39TVL/5135.- [1012]m.- 23-08-2001.- Colectó y donó: M Valladolid.

MNCN 40808.- Pm.- ETC, Manjirón (=Puentes viejas).- UTM 39TVL/5135.- [1012]m.- 23-08-2001.- Colectó y donó: M Valladolid.

MNCN 40809.- Pm.- ETC, Manjirón (=Puentes viejas).- UTM 39TVL/5135.- [1012]m.- 23-08-2001.- Colectó y donó: $\mathrm{M}^{\mathrm{a}}$ Valladolid.

MNCN 40810.- Pm.- ETC, Manjirón (=Puentes viejas).- UTM 39TVL/5135.- [1012]m.- 23-08-2001.- Colectó y donó: $\mathrm{M}^{\mathrm{a}}$ Valladolid.

MNCN 41872.- Pm.- ECF.- Manjirón (=Puentes viejas).- UTM 39TVL/5135.- [1012]m.- 23-08-2001.- Colectó y donó: $M^{\text {a }}$ Valladolid.

Psammodromus hispanicus Fitzinger, 1826

MNCN 7864.- JV.- ECF.- Castillejo, Aranjuez.- UTM: VK/3722.- [515]m. 20-03-1932.- Colectó y donó: E. Morales-Agacino.

MNCN 7875 - 7876.- 2JV.- ECF.- Castillejo, Aranjuez.- UTM: VK/3722. [515]m.- 20-03-1932.- Colectó y donó: E. Morales-Agacino.

MNCN 7885 - 7886.- 2Ad.- ECF.- Cercedilla.- UTM: VL/1010.- [1188]m.

MNCN 7887 - 7888.- 2Ad.- ECF.- Móstoles.- UTM: VK/2664.- [661]m. [01-02-1914].

OBSERVACIONES: Recogidos en el «Libro de registro de entradas de ejemplares en la colección de Zoología, sección de Osteozoología 19111932» pp 41, (AMNCN, 1911).

MNCN 7908.- Ad.- ECF.- Majadahonda.- UTM: VK/2680.- [743]m.- 0205-1976.- Colectó y donó: E. Ortíz.

MNCN 16486.- Ad.- EDC, Móstoles.- UTM: VK/2664.- [661]m.- 25-061982.- Colectó y donó: M. García París.

MNCN 16487.- Ad.- EDC.- Laguna de Ontígola, Aranjuez.- UTM VK/4930.- [560]m.- 07-04-1983.- Colectó y donó: J. Dorda.

MNCN 16488.- Ad.- EDC, Aranjuez.- UTM: VK/4832.- [494]m.- 20-031983.- Colectó y donó: J. Dorda.

MNCN 16490.- Ad.- EDP.- Laguna de Ontigola, Aranjuez.- UTM: VK/4930 - [560]m. - 07-04-1983- Colectó y donó: J. Dorda.

MNCN 16491.- Ad.- EDP.- Colmenar Viejo.- UTM: VL/3501.- [883]m.08-07-1982.- Colectó y donó: L. Dominguez.

MNCN 36404.- ECF.- Villanueva de la Cañada.- UTM: VK/1578. [652]m.- 02-06-1974.- Donó: C. A. de Madrid (U.Z.A.)

MNCN 36405 - 36414.- 10(Ad+Jv).- ECF.- Ciudad Deportiva La Dehesa (Cuatrovientos), Madrid.- UTM: VK/3270.- [670]m.- 13-10-1977.Donó: C. A. de Madrid (U.Z.A.)

MNCN 36415 - 36416.- 2Ad.- ECF.- Galapagar.- UTM: VK/1592.[881]m.- 06-05-1971.- Donó: C. A. de Madrid (U.Z.A.)

MNCN 36437 - 36451.- 16(Ad+Jv).- ECF.- Ciudad Deportiva La Dehesa (Cuatrovientos), Madrid.- UTM: VK/3270.- [670]m.- 22-03-1981.Donó: C. A. de Madrid (U.Z.A.).

MNCN 36462 - 36463.- 2Ad.- ECF.- Ciudad Deportiva La Dehesa (Cuatrovientos), Madrid.- UTM: VK/3270.- [670]m.- 06-05-1980. Donó: C. A. de Madrid (U.Z.A.).

MNCN 36464.- Ad.- ECF.- Majadahonda.- UTM: VK/2680.- [743]m.- $27-$ 03-1974.- Donó: C. A. de Madrid (U.Z.A.).

MNCN 36465.- Ad.- ECF.- Villaviciosa de Odón.- UTM: VK/2368. [650]m.- 04-05-1971.- Donó: C. A. de Madrid (U.Z.A.)

MNCN 36484 - 36490.- 7Ad.- ECF.- Madrid.- UTM: VK/47.- [655]m.- 05-1980.- Donó: C. A. de Madrid (U.Z.A.)

MNCN 36570.- Ad.- ECF.- Cercanías de Madrid.- 28-05-1972.- Donó: C. A. de Madrid (U.Z.A.)

MNCN 36571 - 36577.- 7(Ad+Jv).- ECF.- Zona Sur de Madrid.- 23-101976.- Donó: C. A. de Madrid (U.Z.A.).

MNCN 36578 - 36588.- 11(Ad+Jv).- ECF.- Zona Sur de Madrid.- 12-111977.- Donó: C. A. de Madrid (U.Z.A.).

MNCN 36589.- Ad.- ECF.- Ctra. M-512, entre, Navas del Rey y Robledo de Chavela.- UTM: UK/97.- 11-05-1973.- Donó: C. A. de Madrid (U.Z.A.)

MNCN 36590.- Ad.- ECF.- Venta de la Rubia, Alcorcón.- UTM: VK/3069. [700]m.- 19-10-1975.- C. A. de Madrid (U.Z.A.).

MNCN 36591 - 36595.- 5(Ad+Sad).- ECF.- Campo Real.- UTM: VK/6765.- [777]m.- 12-04-1980.- Donó: C. A. de Madrid (U.Z.A.)

MNCN 36596 - 36597.- 2Ad.- ECF.- Chinchón.- UTM: VK/6443.[753]m.- 13-06-1975.- Donó: C. A. de Madrid (U.Z.A.)

MNCN 36598 - 36599.- 2Ad.- ECF.- Colmenar Viejo.- UTM: VL/3501.[883]m.- 19-02-1970.- Donó: C. A. de Madrid (U.Z.A.)
MNCN 36600.- Sad.- ECF.- Colmenar Viejo.- UTM: VL/3501.- [883]m.28-04-1970.- Donó: C. A. de Madrid (U.Z.A.)

MNCN 36601.- Jv.- ECF.- Colmenar Viejo.- UTM: VL/3501.- [883]m.- 1010-1970.- Donó: C. A. de Madrid (U.Z.A.).

MNCN 36602.- Ad.- ECF.- Hoyo de Manzanares.- UTM: VK/2397.[1001]m.- 08-04-1980.- Donó: C. A. de Madrid (U.Z.A.).

MNCN 36604.- Ad.- ECF.- Madrid.- UTM: VK/47.- [655]m.- 28-101979.- Donó: C. A. de Madrid (U.Z.A.).

MNCN 36605.- Ad.- ECF.- Ciudad Universitaria, Madrid.- UTM: VK/37.[600]m.- 08-05-1978.- Donó: C. A. de Madrid (U.Z.A.).

MNCN 36607.- Ad.- ECF.- E.T.S.I. Montes (Ciudad Universitaria), Madrid.UTM: VK/3878.- [620]m.- 16-09-1972.- Donó: C. A. de Madrid (U.Z.A.).

MNCN 36608 - 36610.- 3Ad.- ECF.- El Pardo, Madrid.- UTM: VK/3486.[620-720]m.- 26-10-1977.- Donó: C. A. de Madrid (U.Z.A.).

MNCN 36611.- Ad.- ECF.- Entrevías, Madrid.- UTM: VK/4270.- [600]m.21-04-1970.- Donó: C. A. de Madrid (U.Z.A.)

MNCN 36612.- Ad.- ECF.- Mingorrubio, Madrid.- UTM: VK/3387.[600]m.- 03-05-1981.- Donó: C. A. de Madrid (U.Z.A.)

MNCN 36613.- Ad.- ECF.- Calle de Fuente de la Peña, Madrid.- UTM: VK/4774.- [700]m.- 27-03-1971.- Donó: C. A. de Madrid (U.Z.A.)

MNCN 36614.- Ad.- ECF.- Vallecas, Madrid.- UTM: VK/4770.- [627]m.01-03-1970.- Donó: C. A. de Madrid (U.Z.A.).

MNCN 36615.- Ad.- ECF.- Museo de América (Ciudad Universitaria), Madrid.- UTM: VK/3877.- [615]m.- 28-03-1970.- Donó: C. A. de Madrid (U.Z.A.).

MNCN 36616.- Ad.- ECF.- Miraflores de la Sierra.- UTM: VL/3518.[1150]m.- 25-04-1970.- Donó: C. A. de Madrid (U.Z.A.).

MNCN 36617.- Ad.- ECF.- Moralzarzal.- UTM: VL/1704.- [979]m.- 2507-1981.- Donó: C. A. de Madrid (U.Z.A.).

MNCN 36618 - 36620.- 3Ad.- ECF.- Móstoles.- UTM: VK/2664.[661]m.- 15/20-09-1970.- Donó: C. A. de Madrid (U.Z.A.)

MNCN 36621.- Ad.- ECF.- San Fernando de Henares.- UTM: VK/5475.[585]m.- 15-08-1976.- Donó: C. A. de Madrid (U.Z.A.).

MNCN 36622.- Ad.- ECF.- Embalse de los Peñascales, Torrelodones.- UTM: VK/2392.- [740-760]m.- 12-05-1974.- Donó: C. A. de Madrid (U.Z.A.)

MNCN 36623 - 36624.- Ad/JV.- ECF.- Torrelodones.- UTM: VK/2192.845]m.- 20-04-1973.- Donó: C. A. de Madrid (U.Z.A.)

MNCN 37308.- Ad.- ECF.- Chinchón.- UTM: VK/6443.- [753]m.- 08-051975.- Donó: C. A. de Madrid (U.Z.A.)

MNCN 37309.- Ad.- ECF.- ¿Valtravieso? (El Pardo), Madrid.- UTM: VK/38.- 22-04-1979.- Donó: C. A. de Madrid (U.Z.A.).

MNCN 39722.- Ad.- ECF.- Navas del Rey.- UTM: UK/9371.- [709]m.- 0605-1985.- Donó: C. A. de Madrid (U.Z.A.).

MNCN 39771.- Ad.- ECF.- Collado-Villalba.- UTM: VK/1699.- [917]m.S. Santa 1981.- Donó: C. A. de Madrid (U.Z.A.).

MNCN 40133.- Hu.- ECF.- Manzanares El Real.- UTM: VL/2708.- [908]m.23-06-1973.- Colectó: J. Ibañez.- Donó: C. A. de Madrid (U.Z.A.).

Familia Scincidae Oppel, 1811

Chalcides bedriagai (Boscá, 1880)

MNCN 37196.- ECF.- Miraflores de la Sierra.- UTM: VL/3518.- [1150]m.20-08-1971.- Donó: C. A. de Madrid (U.Z.A.)

MNCN 37197.- ECF.- La Pedriza, Manzanares El Real.- UTM: VL/21.[1400-1700]m.- 28-02-1971.- Donó: C. A. de Madrid (U.Z.A.).

MNCN 37206.- Ad.- ECF.- Navas del Rey.- UTM: UK/9371.- [709]m.- 2605-1985.- Donó: C. A. de Madrid (U.Z.A.).

MNCN 37207.- Ad.- ECF.- Monte de Abantos, El Escorial.- UTM: VK/0296.- [1754]m.- 22-04-1979.- Donó: C. A. de Madrid (U.Z.A.)

Chalcides striatus Cuvier, 1829

MNCN 5214 - 5215.- 2Ad.- ECF.- San Martín de la Vega.- UTM: VK/5151.- [515]m.- [02-05-1914].- Colectó y donó: F. Árias. OBSERVACIONES: Recogidos en el «Libro de registro de entradas de ejemplares en la colección de Zoología, sección de Osteozoología, 19111932» pp 50. como «C. lineatus», (AMNCN, 1911).

MNCN 5219 - 5220.- 2Ad.- ECF.- El Ventorrillo, Mancomunidad de Cercedilla y Navacerrada.- UTM: VL/1312.- [1500]m.- -08-1918.Colectó y donó: A. Cabrera.

MNCN 5232.- Ad.- ECF.- El Escorial.- UTM: VK/0493.- [909]m.

MNCN 5233.- ECF.- Cercedilla.- UTM: VL/1010.- [1188]m.- 05-06-1926.

MNCN 37230.- Jv.- ECF.- Valdemoro.- UTM: VK/4249.- [615]m.- 05-051976.- Donó: C. A. de Madrid (U.Z.A.).

MNCN 37231.- Jv.- ECF.- Pelayos de la Presa.- UTM: UK/8668.- [570]m.25-05-1977.- Donó: C. A. de Madrid (U.Z.A.) 
MNCN 37252 - 37254.- 3Ad.- ECF.- Moralzarzal.- UTM: VL/1704.[979]m.- 25-07-1981.- Donó: C. A. de Madrid (U.Z.A.).

MNCN 37262.- Jv.- ECF.- Tablada, Guadarrama.- UTM: VL/0406. [1340]m.- 26-06-1969.- Donó: C. A. de Madrid (U.Z.A.).

MNCN 37263.- Jv.- ECF.- Montejo de la Sierra.- UTM: VL/5545.[1148]m.- 22-08-1971.- Donó: C. A. de Madrid (U.Z.A.).

MNCN 37264.- Ad.- ECF.- Embalse de El Vellón, Guadalix de la Sierra. UTM: VL/41.- [828]m.- 12-04-1973.- Donó: C. A. de Madrid (U.Z.A.).

Suborden Serpentes Linnaeus, 1758

Familia Colubridae Oppel, 1811

Hemorrhois hippocrepis (Linnaeus, 1758)

MNCN 8754.- Jv.- ECF.- Cenicientos.- UTM: UK/7558.- [774]m.- <[2104-1933].- Colectó y donó: [A. Izquierdo].

OBSERVACIONES: Recogido en el "Libro de registro de entradas de ejemplares en la colección de Zoología, sección de Osteozoología, 19311939” pp 10, como Zamenis hippocrepis (AMNCN, 1931).

Coronella austriaca Laurenti, 1768

MNCN 6419.- Sad.- ECF.- Navacerrada.- UTM: VL/1409.- [1203]m.- 0107-1980.- Colectó y donó: J. Mellado.

MNCN 6420.- Ad.- ECF.- Puerto de Los Cotosc (=del Paular), Rascafría. UTM: VL/1919.- [1830]m.- 26-07-1980.- Colectó y donó: J. Mellado.

MNCN 8300.- Ad.- ECF.- [Monasterio de] El Paular, Rascafría.- UTM VL/2527.- [1159]m.- 02-06-1980.- Colectó y donó: J. Mellado.

MNCN 8732.- Ad.- ECF.- El Ventorrillo, Mancomunidad de Cercedilla y Navacerrada.- UTM: VL/1312.- [1500]m.- -08-1918.- Colectó y donó A. Cabrera.

MNCN 8736.- Ad.- ECF.- Cercedilla.- UTM: VL/1010.- [1188]m.- 08 1918.

MNCN 8738.- Ad.- ECF.- El Ventorrillo, Mancomunidad de Cercedilla y Navacerrada.- UTM: VL/1312.- [1500]m.- Colectó: J. Hernández.

MNCN 8742.- Ad.- ECF.- El Ventorrillo, Mancomunidad de Cercedilla y Navacerrada.- UTM: VL/1312.- [1500]m.- 05-1934.

MNCN 39410.- Ad.- ECF.- Subiendo hacia la Bola del Mundo, desde la Pedriza.- 26-08-1971.- Donó: C. A. de Madrid (U.Z.A.)

MNCN 39538.- Ad.- ECF.- Montejo de la Sierra.- UTM: VL/5545.[1148]m.- 21-04-1971.- Donó: C. A. de Madrid (U.Z.A.).

MNCN 39545.- Ad.- ECF.- Montejo de la Sierra.- UTM: VL/5545. [1148]m.- 15-07-1972.- Colectó: J. L. Ceresuela.- Donó: C. A. de Madrid (U.Z.A.)

MNCN 39568.- Ad.- ECF.- Refugio Zabala en Peñalara, Rascafría.- UTM VL/1922.- [2300]m.- 03-05-1970.- Colectó: A. Salvador.- Donó: C. A. de Madrid (U.Z.A.)

MNCN 39569.- Jv.- ECF.- Montejo de la Sierra.- UTM: VL/5545.[1148]m.- 25-05-1973.- Donó: C. A. de Madrid (U.Z.A.)

MNCN 39577.- Ad.- ECF.- Pico de la Tornera, Puebla de la Sierra.1865m.- 13-05-1973.- Donó: C. A. de Madrid (U.Z.A.).

MNCN 39582.- Ad.- ECF.- Base del Yelmo (=Peña del Diezmo), La Pedriza, Manzanares El Real.- UTM: VL/2226.- [1600]m.- 26-05-1974.Donó: C. A. de Madrid (U.Z.A.)

Coronella girondica (Daudin, 1803)

MNCN 8688.- Ad.- ECF.- Villa del Prado.- UTM: UK/8959.- [510]m.$<$ [24-11-1912].- Colectó y donó: F. Árias.

OBSERVACIONES: Recogido en el «Libro de registro de entradas de ejemplares en la colección de Zoología, sección de Osteozoología, 19111932» pp 26, (AMNCN, 1911).

MNCN 8713.- Ad.- ECF.- Valdemorillo.- UTM: VK/0894.- [815]m.- 17 10-1976.- Colectó: J. Roset Esteve.

MNCN 8725.- Jv.- ECF.- Robledo de Chavela.- UTM: UK/9584.- [903]m.12-05-1934.- Colectó: C. Bolivar.

MNCN 8726.- Ad.- ECF.- [Estación de FFCC de] Montarco, RibasVaciamadrid.- UTM: VK/5467.- [600]m.- 15-05-1934.- Colectó: D. Peláez.

MNCN 8743.- Ad.- ECF.- El Ventorrillo, Mancomunidad de Cercedilla y Navacerrada.- UTM: VL/1312.- [1500]m.- -07-1933.- Colectó: D. Peláez.

MNCN 9136.- Jv.- ECF.- Horcajuelo de la Sierra.- UTM: VL/5445. [1145]m.- -07-1936.

MNCN 9137.- JV.- ECF.- Peñalara (*), Rascafría.- VL/1922.- Colectó y Donó: C. Bolívar.

OBSERVACIONES: (*) Se encontraba en un frasco en el que había una etiqueta que decía: Lacerta viridis (Peñalara), no había lagarto, pero si otro joven de Natrix maura (MNCN 8578). En cualquier caso, debe tratarse de un error de etiquetación, ya que ni Coronella girondica ni Natrix maura, se distribuyen en Peñalara.

MNCN 16324.- Ad.- PCF - EDP.- Ctra. M-611 de Miraflores al Puerto de la Morcuera, Miraflores de la Sierra.- UTM: VL/32. [1150-1796]m.- 2604-1984.- Colectó y donó: A. García-Valdecasas.

MNCN 16326.- EDP.- La Berzosa, Moralzarzal.- UTM: VK/1995.[900]m.- 14-05-1983.- Colectó y donó: R. R. Talavera \& J. C. Arrechea.

MNCN 17924.- Ad.- ECF.- San Martín de Valdeiglesias.- UTM: UK/8169.[681]m.- verano-1978.- Colectó y donó: C. E. Pérez Santos.

MNCN 17950.- Ad.- ECF.- San Martín de Valdeiglesias.- UTM: UK/8169.[681]m.- -07-1986.- Colectó y donó: C. E. Pérez Santos.

MNCN 39485.- Ad.- ECF.- Bustarviejo.- UTM: VL/4023.- [1222]m.- 2502-1970.- Donó: C. A. de Madrid (U.Z.A.).

MNCN 39486.- Jv.- ECF.- La Navata, Galapagar.- UTM: VK/1694.[840]m.- 29-04-1974.- Donó: C. A. de Madrid (U.Z.A.).

MNCN 39487.- Ad.- ECF.- Horcajuelo de la Sierra.- UTM: VL/5445.[1145]m.- 04-08-1973.- Donó: C. A. de Madrid (U.Z.A.)

MNCN 39488.- Ad.- ECF.- Ciudad Universitaria, Madrid.- UTM: VK/37.[600]m.- 28-04-1975.- Donó: C. A. de Madrid (U.Z.A.).

MNCN 39489 - 39490.- ECF.- La Pedriza, Manzanares El Real.- UTM: VL/21 - [1400-1700]m.- 22-06-1973 - C. A de Madrid (U.Z.A.)

MNCN 39491.- Ad.- ECF.- Montejo de la Sierra.- UTM: VL/5545.[1148]m.- 21-04-1971.- Donó: C. A. de Madrid (U.Z.A.).

MNCN 39492.- Ad.- ECF.- Montejo de la Sierra.- UTM: VL/5545.[1148]m.- 26-06-1971.- Donó: C. A. de Madrid (U.Z.A.)

MNCN 39493.- Ad.- ECF.- San Martín de Valdeiglesias.- UTM: UK/8169.[681]m.- 23-07-1972.- C. A. de Madrid (U.Z.A.)

MNCN 39605.- Ad.- ECF.- Pantano de San Juan, Pelayos de la Presa.- UTM: UK/8869.- [580]m.- verano-1975.- Donó: C. A. de Madrid (U.Z.A.).

MNCN 39606.- Jv.- ECF.- Monte de Santa Catalina, Valdemaqueda.UTM: UK/9187.- [1387]m.- 11-09-1977.

MNCN 39609.- Ad.- ECF.- Puerto de Navafría [=Puerto de Lozoya] (vertiente Sur), Lozoya.- UTM: VL/3137.- (1760)m.- 05-06-1979.

MNCN 39616 - 39619.- Jv.- ECF.- Montejo de la Sierra.- UTM: VL/5545.[1148]m.- 27-03-1971.- Donó: C. A. de Madrid (U.Z.A.)

MNCN 39628.- JV.- ECF.- La Pedriza, Manzanares el Real.- UTM: VL/21.[1400-1700]m.- 18-04-1971.- Donó: C. A. de Madrid (U.Z.A.).

MNCN 39636.- Jv.- ECF.- Galapagar.- UTM: VK/1592.- [881]m.- 29-031975.- Donó: C. A. de Madrid (U.Z.A.).

MNCN 39660.- Ad.- ECF.- Villavieja de Lozoya.- UTM: VL/4339.[1066]m.- 01-06-1969.- Colectó y donó: P. Garzón.

MNCN 39661.- JV.- ECF.- El Escorial.- UTM: VK/0493.- [909]m.- 16-051971.

Rhinechis scalaris (Schinz, 1822)

MNCN 8771.- Ad.- ECF.- El Pardo, Madrid.- UTM: VK/47.- [620-720]m.05-03-1907.- Colectó y donó: J. Árias Encobet.

OBSERVACIONES: Recogido en el «Libro de registro de entradas de objetos a diversas secciones del Museo de Ciencias Naturales, 1907-1913» pp. 2, (AMNCN, 1907).

MNCN 8775.- Ad.- ECF.- El Ventorrillo, Mancomunidad de Cercedilla y Navacerrada.- UTM: VL/1312.- [1500]m.- 1976.- Colectó: R. Agenjo.

MNCN 8781.- Sad.- ECF.- El Escorial.- UTM: VK/0493.- [909]m.Colectó y donó: J. Hita.

MNCN 8782.- Sad.- ECF.- Galapagar.- UTM: VK/1592.- [881]m.- 01-071969.- Colectó y donó: E. Ortíz.

MNCN 8794.- Ad.- ECF.- Arganda del Rey.- UTM: VK/6261.- [618]m.- 06-1964.- Colectó y donó: J. M. Rey Salgado.

MNCN 8795 - 8796.- 2Sad.- ECF.- El Pardo, Madrid.- UTM: VK/3486.[620-720]m.- -1907.- Colectó y donó: J. Árias Encobet.

MNCN 8802.- Sad.- ECF.- Cercedilla.- UTM: VL/1010.- [1188]m.- 16-031969.- Colectó y donó: J. M. Rey Salgado.

MNCN 8811.- Ad.- ECF.- El Pardo, Madrid.- UTM: VK/3486.- [620720]m.- -01-1908.- Colectó y donó: J. Árias Encobet

MNCN 8821 - 8823.- Ad.- ECF.- El Pardo, Madrid.- UTM: VK/3486.[620-720]m.- 23/27-04-1907.- Colectó y donó: J. Árias Encobet.

MNCN 8845 - 8846.- 2Ad.- ECF.- El Pardo, Madrid.- UTM: VK/3486.[620-720]m.- 24-07-1907.- Colectó y donó: J. Árias Encobet.

MNCN 8847.- Ad.- ECF.- El Pardo, Madrid.- UTM: VK/3486.- [620720]m.- 21-05-1907.- Colectó y donó: J. Árias Encobet.

MNCN 8848 - 8849.- Ad/Sad.- ECF.- Robledo de Chavela.- UTM: UK/9584.- [903]m.- 12-05-1934.- Colectó y donó: C. Bolívar. 
MNCN 8850 - 8851.- Sad/Ad.- ECF.- El Pardo, Madrid.- UTM: VK/3486.[620-720]m.- 29-05-1907.- Colectó y donó: J. Árias Encobet.

MNCN 8852.- Sad.- ECF.- El Pardo, Madrid.- UTM: VK/3486.- [620720]m.- 21-05-1907.- Colectó y donó: J. Árias Encobet.

MNCN 8853.- Ad.- ECF.- El Pardo, Madrid.- UTM: VK/3486.- [620720]m.- 27-04-1907.- Colectó y donó: J. Árias Encobet.

MNCN 8854.- Ad.- ECF.- El Pardo, Madrid.- UTM: VK/3486.- [620720]m.- -05-1907.- Colectó y donó: J. Árias Encobet.

MNCN 8855.- Ad.- ECF.- El Pardo, Madrid.- UTM: VK/3486.- [620720]m.- 18-05-1907.- Colectó y donó: J. Árias Encobet.

MNCN 8856.- Ad.- ECF.- El Pardo, Madrid.- UTM: VK/3486.- [620720]m.- 24-05-1907.- Colectó y donó: J. Árias Encobet.

MNCN 8857.- Ad.- ECF.- El Pardo, Madrid.- UTM: VK/3486.- [620 720]m.- 05-05-1907.- Colectó y donó: J. Árias Encobet.

MNCN 8860 - 8861.- 2Ad.- ECF.- El Pardo, Madrid.- UTM: VK/3486. [620-720]m.- -05-1907.- Colectó y donó: J. Árias Encobet.

MNCN 8863.- Ad.- ECF.- El Pardo, Madrid.- UTM: VK/3486.- [620720]m.- 25-04-1907.- Colectó y donó: J. Árias Encobet.

MNCN 8864 - 8866.- 3Ad.- ECF.- El Pardo, Madrid.- UTM: VK/3486. [620-720]m.- 15/24-05-1907.- Colectó y donó: J. Árias Encobet.

MNCN 9050.- Ad.- ECF.- El Escorial.- UTM: VK/0493.- [909]m.- -021885.- Colectó y donó: F.de P. Martinez y Sáez.

MNCN 9145 - 9146.- JV/Sad.- ECF.- El Escorial.- UTM: VK/0493.[909]m.- Colectó y donó: Lauffer.

MNCN 9152.- Ad.- ECF.- Fuente del Fresno, San Sebastián de los Reyes. UTM: VK/5093.- [650]m.- 30-03-1980.- Colectó y donó: M. L. Esteban.

MNCN 9166.- Ad.- ECF.- Valdemorillo.- UTM: VK/0894.- [815]m.- 21 05-1986.- Colectó y donó: A. Mújica Nava.

MNCN 9176.- Sad.- ECF.- San Martín de Valdeiglesias.- UTM: UK/8169.[681]m.- 28-06-1986.- Colectó y donó: C. E. Pérez Santos.

MNCN 10023.- Jv.- ECF.- San Cristobal de Los Angeles, Madrid.- UTM VK/4166.- [580]m.- 17-04-1983.- Colectó y donó: A. Fernández Lop.

MNCN 16197.- Jv.- ECF.- Estación depuradora de Rejas (Barajas), Madrid.- UTM: VK/5478.- [560]m.- 19-07-1990.- Colectó y donó: J. C. Rey Fraile.

MNCN 16333.- Ad.- PCF - EDP.- Ctra. [M-607] de El Ventorrillo a Colmenar Virjo, Cercedilla.- -1983.- Colectó y donó: J. C. Blanco.

MNCN 16336.- Ad.- PCF - EDP.- Presa del Embalse de Pinilla (=Lozoya), Lozoya.- UTM: VL/3433.- [1098]m.- 23-06-1983.- Colectó y donó: A. Mújica Nava.

MNCN 16337.- Ad.- PCF - EDP.- El Ventorrillo, Mancomunidad de Cercedilla y Navacerrada.- UTM: VL/1312.- [1500]m.- 12-07-1983. Colectó y donó: A. Larramendi.

MNCN 16342.- PCF - EDC.- Arroyo del Pradejón, Navalagamella.- UTM: VK/0485.- 750m.- 17-10-1982.- Colectó y donó: A. Mújica Nava.

MNCN 16343.- Ad.- PCF - EDP, San Agustín de Guadalix.- UTM: VL/4703.[684]m.- 15-04-1983.- Colectó y donó: R. R. Talavera \& J. C. Arrechea.

MNCN 17941.- JV.- ECF.- San Martín de Valdeiglesias.- UTM: UK/8169.[681]m.- -09-1983.- Colectó y donó: C. E. Pérez Santos.

MNCN 18726.- Ad.- ECF.- Villamantilla.- UTM: VK/0466.- [551]m.- 27 05-1990.- Colectó y donó: Srs. de Pérez Olea.

MNCN 19618.- PCF - EDP, Fresno de Torote.- UTM: VK/6593.- [657]m.Colectó y donó: A. Mújica Nava.

MNCN 19695.- EDC.- Embalse de El Atazar.- UTM: VL/52.- [870]m.- 25 06-1988.- Colectó y donó: M. García París \& C. Martín.

MNCN 19922.- Jv.- ECF.- Cocina del edificio Kodak, Km 23 de la N-VI, Las Rozas de Madrid.- UTM: VK/2487.- [750]m.- 07-06-1995.- Colectó y donó: A. Castell.

MNCN 20032.- Ad.- ECF.- Brunete.- UTM: VK/1573.- [656]m.- 08-081979.- Colectó y donó: F. Hiraldo.

MNCN 20033.- Ad.- ECF.- Cerceda, El Boalo.- UTM: VL/2105.- [949]m.15-08-1981.- Colectó y donó: J. C. Blanco \& J. Delibes.

MNCN 20036.- Ad.- ECF.- Navacerrada.- UTM: VL/1409.- [1203]m.- 1006-1981.- Colectó y donó: J. Delibes.

MNCN 20749.- Jv.- ECF.- Las Matas, Las Rozas de Madrid.- UTM: VK/2489.- [750]m.- 15-06-1996.- Colectó: P. Muñecas

MNCN 24280.- Ad.- ECF.- Urbanización «El Bosque», Villaviciosa de Odón.- UTM: VK/27.- [600-650]m.- 12-05-1997.- Colectó y donó: I. Doadrío \& A. Corcuera.

MNCN 37606.- Ad.- ECF.- Camarma de Esteruelas.- UTM: VK/6888. [640]m.- 29-03-1981.- Donó: C. A. de Madrid (U.Z.A.).

MNCN 37685.- Ad.- ECF.- Aranjuez.- UTM: VK/4832.- [494]m.- 18-061974.- Donó: C. A. de Madrid (U.Z.A.).
MNCN 37686.- Ad.- ECF.- Brunete.- UTM: VK/1573.- [656]m.- 11-121971.- Colectó: Mariví..- Donó: C. A. de Madrid (U.Z.A.).

MNCN 37688.- Sad.- ECF.- Brunete.- UTM: VK/1573.- [656]m.- 08-061980.- Donó: C. A. de Madrid (U.Z.A.)

MNCN 37689.- Sad- ECF- Collado Mediano- UTM: VL/1305. [1020]m.- 02-10-1974.- Donó: C. A. de Madrid (U.Z.A.).

MNCN 37690.- JV.- ECF.- Mataelpino, El Boalo.- UTM: VL/2010.1100]m.- 08-06-1975.- Donó: C. A. de Madrid (U.Z.A.)

MNCN 37691.- Sad.- ECF.- Guadarrama.- UTM: VL/0803.- [981]m.- 20-051974.- Colectó: J. Gisber \& J. Melendro.- Donó: C. A. de Madrid (U.Z.A.).

MNCN 37692.- Sad.- ECF.- Guadarrama.- UTM: VL/0803.- [981]m.- 2505-1974.- Donó: C. A. de Madrid (U.Z.A.).

MNCN 37693.- Sad.- ECF.- Horcajuelo de la Sierra.- UTM: VL/5445.[1145]m.- 28-07-1973.- Donó: C. A. de Madrid (U.Z.A.).

MNCN 37694.- Sad.- ECF.- Horcajuelo de la Sierra.- UTM: VL/5445.[1145]m.- 05-08-1973.- Donó: C. A. de Madrid (U.Z.A.)

MNCN 37695.- Ad.- ECF.- Horcajuelo de la Sierra.- UTM: VL/5445.[1145]m.- 04-08-1974.- Donó: C. A. de Madrid (U.Z.A.)

MNCN 37696.- Sad.- ECF.- Horcajuelo de la Sierra.- UTM: VL/5445.[1145]m.- 30-07-1976.- Donó: C. A. de Madrid (U.Z.A.).

MNCN 37697.- JV.- ECF.- Horcajuelo de la Sierra.- UTM: VL/5445.[1145]m.- 04-08-1976.- Donó: C. A. de Madrid (U.Z.A.).

MNCN 37698.- Ad.- ECF.- Madrid.- UTM: VK/47.- [655]m.- 12-061980.- Donó: C. A. de Madrid (U.Z.A.).

MNCN 37699.- Sad.- ECF.- Aravaca, Madrid.- UTM: VK/3379.- [647]m.01-06-1972.- Colectó: J. L. Ceresuela.- Donó: C. A. de Madrid (U.Z.A.).

MNCN 37700.- Ad.- ECF.- Carretera Barajas, Madrid.- UTM: VK/58.- [680700]m.- 15-06-1975.- Colectó: C. Pérez.- Donó: C. A. de Madrid (U.Z.A.).

MNCN 37701.- Sad.- ECF.- M-618 (=Ctra de Torrelodones a Hoyo de Manzanares), Madrid.- UTM: VK/3486.- [840-1000]m.- 15-12-1979.Colectó: C. G. López.- Donó: C. A. de Madrid (U.Z.A.)

MNCN 37702.- Ad.- ECF.- Monte de El Pardo, Madrid.- UTM: VK/38.[620-720]m.- 08-05-1980.- Colectó: C. Rodrígez Vigal \& J. González.Donó: C. A. de Madrid (U.Z.A.)

MNCN 37703.- Ad.- ECF.- El Pardo, Madrid.- UTM: VK/3486.- [620720]m.- 06-07-1980.- Colectó: L. M. Gonzalez \& C. Rodríguez Vigal.Donó: C. A. de Madrid (U.Z.A.)

MNCN 37704.- Ad.- ECF.- La Pedriza, Manzanares El Real.- UTM: VL/21.- [1400-1700]m.-- 05-1971.- Donó: C. A. de Madrid (U.Z.A.).

MNCN 37705.- Jv.- ECF.- Miraflores de la Sierra.- UTM: VL/3518.[1150]m.- 02-07-1973.- Colectó: Timoteo Sanz.- Donó: C. A. de Madrid (U.Z.A.).

MNCN 37706.- Sad.- ECF.- Montejo de la Sierra.- UTM: VL/5545.[1148]m.- -05-1969.- Donó: C. A. de Madrid (U.Z.A.)

MNCN 37707.- Ad.- ECF.- Montejo de la Sierra.- UTM: VL/5545.- [1148]m.-06-1969.- Colectó: J. Garzón.- Donó: C. A. de Madrid (U.Z.A.).

MNCN 37708.- Sad.- ECF.- Montejo de la Sierra.- UTM: VL/5545.[1148]m.- 22-08-1971.- Donó: C. A. de Madrid (U.Z.A.)

MNCN 37709.- Ad.- ECF.- Montejo de la Sierra.- UTM: VL/5545.- [1148]m.07-1972.- Colectó: J. L. Ceresuela.- Donó: C. A. de Madrid (U.Z.A.)

MNCN 37710.- Jv.- ECF- Montejo de la Sierra- UTM: VL/5545. [1148]m.- Verano-1972.- Donó: C. A. de Madrid (U.Z.A.).

MNCN 37711.- Ad.- ECF.- Montejo de la Sierra.- UTM: VL/5545.[1148]m.- 23-07-1973.- Donó: C. A. de Madrid (U.Z.A.)

MNCN 37712.- Ad.- ECF.- Moralzarzal.- UTM: VL/1704.- [979]m.- 0306-1972.- Colectó: C. Correa.- Donó: C. A. de Madrid (U.Z.A.)

MNCN 37713.- Ad.- ECF.- ¿Los Pozos?, Navalcarnero.- 15-05-1975.- C. A. de Madrid (U.Z.A.)

MNCN 37714.- Ad.- ECF.- Papelera del Centro (Río Guadarrama), Navalcarnero.- UTM: VK/1961.- [550]m.- 25-07-1975.- Donó: C. A. de Madrid (U.Z.A.).

MNCN 37715.- Sad.- ECF.- Papelera del Centro (Río Guadarrama), Navalcarnero.- UTM: VK/1961.- [550]m.- 14-08-1975.- Donó: C. A. de Madrid (U.Z.A.).

MNCN 37716.- Ad.- ECF.- La Pedriza, Manzanares El Real.- UTM: VL/21.- [1400-1700]m.- 22-06-1971.- Donó: C. A. de Madrid (U.Z.A.).

MNCN 37717.- Ad.- ECF.- Río Lozoya cruce con el puente de Taboada, Gargantilla del Lozoya.- UTM: VL/3032.- [1000]m.- 23-05-1976.Donó: C. A. de Madrid (U.Z.A.).

MNCN 37718 - 37719.- 2Ad.- ECF.- Finca de los Ángeles, San Martín de la Vega.- UTM: VK/5457.- [540]m.- 02/04-06-1973.- Donó: C. A. de Madrid (U.Z.A.). 
MNCN 37720.- Sad.- ECF.- San Martín de la Vega.- UTM: VK/5151.[515]m.- 15-05-1974.- Colectó: M. Cuesta Pons.- Donó: C. A. de Madrid (U.Z.A.).

MNCN 37721.- Jv.- ECF.- San Martín de la Vega.- UTM: VK/5151. [515]m.- 04-06-1975.- Donó: C. A. de Madrid (U.Z.A.)

MNCN 37722.- Sad.- ECF.- San Martín de la Vega.- UTM: VK/5151. [515]m.- <[1991].- Colectó: L. Cuesta.- Donó: C. A. de Madrid (U.Z.A.)

MNCN 37723.- Sad.- ECF.- Torrelaguna.- UTM: VL/5420.- [744]m.- 2805-1970.- Donó: C. A. de Madrid (U.Z.A.).

MNCN 37724.- Ad.- ECF.- Embalse de los Peñascales, Torrelodones.UTM: VK/2392.- [740-760]m.- 17-06-1975.- Donó: C. A. de Madrid (U.Z.A.).

MNCN 37725.- Jv.- ECF.- Valdemorillo.- UTM: VK/0894.- [815]m.- 1705-1975.- Donó: C. A. de Madrid (U.Z.A.).

MNCN 37726.- Ad.- ECF.- Valdemorillo.- UTM: VK/0894.- [815]m.- 0406-1978.- Donó: C. A. de Madrid (U.Z.A.)

MNCN 37727.- Ad.- ECF.- Villanueva de la Cañada.- UTM: VK/1578. [652]m.- 24-04-1973.- Colectó: J.L. Ceresuela.- Donó: C. A. de Madrid (U.Z.A.).

MNCN 37728.- Ad.- ECF.- Villanueva de la Cañada.- UTM: VK/1578.[652]m.- 02-05-1973.- Donó: C. A. de Madrid (U.Z.A.).

MNCN 37729.- Ad.- ECF.- Villanueva de la Cañada.- UTM: VK/1578. [652]m.- 03-06-1973.- Donó: C. A. de Madrid (U.Z.A.).

MNCN 37848.- Sad.- ECF.- Manzanares El Real.- UTM: VL/2708. [908]m.- 24-05-1981.- Colectó: J. Yerte.- Donó: C. A. de Madrid (U.Z.A.).

MNCN 39518.- Sad.- ECF.- Puerta de Hierro, Madrid.- UTM: VK/3879.[590]m.- 07-06-1979.- Donó: C. A. de Madrid (U.Z.A.).

MNCN 39519.- Sad.- ECF.- Los Santos de la Humosa.- UTM: VK/7883.[880]m.- 14-11-1983.- Donó: C. A. de Madrid (U.Z.A.).

MNCN 40852.- EDC.- Procede del A.M.A. Butrago de Lozoya.- 31-071997.

Macroprotodon brevis (Günther, 1862)

MNCN 8428.- Jv.- ECF.- Casa de Campo, Madrid.- UTM: VK/37.- [600650]m.- 06-1964

MNCN 8733.- Ad.- ECF.- Cercedilla.- UTM: VL/1010.- [1188]m.- $<[11$ 09-1914].

OBSERVACIONES: Pese a tener $\mathrm{n}^{\circ}$ de entrada 1914.09.11.04, no está Recogido en el «Libro de registro de entradas de ejemplares en la colección de Zoología, sección de Osteozoología, 1911-1932» pp 71, por no existir anotaciones, en dicho libro, entre el 13 de julio y el 24 de septiembre, (AMNCN, 1911)

MNCN 16345.- Ad.- EDC, San Martín de Valdeiglesias.- UTM: UK/8169. [681]m.- 05-04-1980.- Colectó y donó: C. E. Pérez Santos \& D. Pérez Imobersteg.

Malpolon monspessulanus (Herman, 1840)

MNCN 8829.- Jv.- ECF.- Ciudad Universitaria, Madrid.- UTM: VK/37.[600]m.- 24-05-1968.

MNCN 8877.- JV.- ECF.- Las Rozas de Madrid.- UTM: VK/2582.[718]m.- 06-07-1975.

MNCN 8882.- Jv.- ECF.- El Escorial.- UTM: VK/0493.- [909]m.- Colectó y donó: Sr. Gaspar

MNCN 8888.- Sad.- ECF.- El Pardo, Madrid.- UTM: VK/3486.- [620720]m.- -05-1907.- Colectó y donó: J. Árias Encobet.

MNCN 8917.- Ad.- ECF.- El Pardo, Madrid.- UTM: VK/3486.- [620720]m.- 01-05-1907.- Colectó y donó: J. Árias Encobet.

MNCN 8920.- Ad.- ECF.- Campo Real.- UTM: VK/6765.- [777]m.- 1912.Colectó y donó: F. Árias.

MNCN 8940 - 8943.- 2Sad/2Ad.- ECF.- El Pardo, Madrid.- UTM VK/3486.- [620-720]m.- 04-1907.- Colectó y donó: J. Árias Encobet.

MNCN 8948 - 8949.- 2Sad.- ECF.- El Pardo, Madrid.- UTM: VK/3486. [620-720]m.- 01/21-05-1907.- Colectó y donó: J. Árias Encobet.

MNCN 8951 - 8953.- 3Sad.- ECF.- El Pardo, Madrid.- UTM: VK/3486.[620-720]m.- 05/27-05-1907.- Colectó y donó: J. Árias Encobet.

MNCN 9157.- Sad.- ECF.- Urbanización «El Bosque», Villaviciosa de Odón. UTM: VK/27.- [600-650]m.- 10-05-1977.- Colectó y donó: I. Doadrio.

MNCN 9161.- Jv.- ECF.- Arganda del Rey.- UTM: VK/6261.- [618]m.- 1978.- Colectó y donó: M. L. Esteban.

MNCN 9162.- ECF.- Ctra. M-104 (= M-606) a 5 Km de, Colmenar Viejo.UTM: VL/4003.- [840-860]m.- 29-05-1986.- Colectó: R. Márquez. Donó: R. Márquez.
MNCN 9164.- Ad.- ECF.- Valdemorillo.- UTM: VK/0894.- [815]m.- 2105-1986.- Colectó y donó: A. Mújica Nava.

MNCN 16347.- EDC.- Húmera, Pozuelo de Alarcón.- UTM: VK/3375.[660]m.- 02-05-1982.- Colectó y donó: M. García París.

MNCN 16351.- Ad.- PCF - EDP, Villaviciosa de Odón.- UTM: VK/2368.650]m.- 14-06-1982.- Colectó y donó: I. Doadrío.

MNCN 16352.- Ad.- PCF - EDP, Villaviciosa de Odón.- UTM: VK/2368.[650]m.- 26-06-1982.- Colectó y donó: I. Doadrio \& P. Garzón.

MNCN 16355.- EDC, Villaviciosa de Odón.- UTM: VK/2368.- [650]m.19-09-1982.- Colectó y donó: I. Doadrío.

MNCN 16356.- EDC, Chapinería.- UTM: UK/9770.- [680]m.- 18-021984 - Colectó y donó: M. García París.

MNCN 16358.- Ad.- PCF - EDP.- Las Matas, Las Rozas de Madrid.- UTM: VK/2489.- [750]m.- 25-04-1983.- Colectó y donó: A. Mújica Nava.

MNCN 16361.- Ad.- PCF - EDP, Manzanares El Real.- UTM: VL/2708.[908]m.- 14-10-1984.- Colectó y donó: D. Caballero.

MNCN 16365.- Ad.- PCF - EDP, San Agustín de Guadalix.- UTM: VL/4703.684]m.- 19-04-1984.- Colectó y donó: R. R. Talavera \& J. C. Arrechea.

MNCN 16366.- Ad.- PCF - EDP.- Ctra. M-607 de Colmenar Viejo a El Ventorrillo.- UTM: VL/20.- 1983.- Colectó y donó: J. C. Blanco.

MNCN 16367.- Ad.- PCF - EDP, Villamantilla.- UTM: UK/0466.551]m.- 24-06-1984.- Colectó y donó: Srs. de Pérez Olea

MNCN 16368.- Ad.- PCF - EDP, Quijorna.- UTM: VK/1075.- [573]m.- 2706-1984.- Colectó y donó: A. Mújica Nava.

MNCN 16369.- Ad.- PCF - EDP, El Escorial.- UTM: VK/0493.- [909]m.- 0710-1984.- Colectó y donó: M. Ga París, C. Martín \& A. García Martínez.

MNCN 16372.- Ad.- EDP.- Río Jarama, Madrid.- UTM: VK/59.- [540]m.1982.

MNCN 18727.- ECF.- Villamantilla.- UTM: VK/0466.- [551]m.- 27-051990.- Colectó y donó: Srs. de Pérez Olea.

MNCN 23910.- Ad.- ECF.- Cerceda, El Boalo.- UTM: VL/2105.- [949]m.04-07-1989.

MNCN 24570.- Ad.- ECF.- Soto del Real.- UTM: VK/4585.- [921]m.- 1105-1983.- Colectó y donó: I. Esteban.

MNCN 35425.- Ad.- ECF.- Valdemorillo.- UTM: VK/0894.- [815]m.- 1503-1981.- Colectó: A. Mugica.- Donó: C. A. de Madrid (U.Z.A.).

MNCN 35426.- Jv.- ECF.- 04-1974.- Donó: C. A. de Madrid (antigua Unidad de Zoología Aplicada).

MNCN 35427.- Sad.- ECF.- Torrejón de Ardoz de Velasco.- [589][m.- 0304-1973.- Colectó: P. Muñoz.- Donó: C. A. de Madrid (U.Z.A.).

MNCN 37985.- Sad.- ECF.- 07-06-1975.- Donó: C. A. de Madrid (U.Z.A.).

MNCN 37986.- Ad.- ECF.- 09-1979.- Donó: C. A. de Madrid (U.Z.A.)

MNCN 37987 - 37989.- 3Ad.- ECF.- Carretera entre San Martín y Villaviciosa de Odón.- 07-06-1975.- Donó: C. A. de Madrid (U.Z.A.).

MNCN 37992.- ECF.- «El Encín», Alcalá de Henares.- UTM: VK/4744.[610]m.- 12-06-1987.- Colectó: J.L. Rubio.- Donó: C. A. de Madrid (U.Z.A.).

MNCN 37993.- Jv.- ECF.- Alcorcón.- UTM: VK/2966.- [718]m.- 19-101978.- Colectó: B.Elvira.- Donó: C. A. de Madrid (U.Z.A.).

MNCN 37994.- Ad.- ECF.- Barajas, Madrid.- UTM: VK/5180.- [622]m.08-05-1973.- Donó: C. A. de Madrid (U.Z.A.).

MNCN 37995.- Ad.- ECF.- Barajas, Madrid.- UTM: VK/5180.- [622]m.- 05-1980.- Donó: C. A. de Madrid (U.Z.A.).

MNCN 37996.- Ad.- ECF.- Los Pozuelos, Manzanares El real.- UTM: VL/2305.- [920-980]m.- 02-05-1982.- Colectó: Rodrigo Fernández.Donó: C. A. de Madrid (U.Z.A.).

MNCN 37997.- Ad.- ECF.- Cerceda, El Boalo.- UTM: VL/2105.- [949]m.11-06-1979.- Donó: C. A. de Madrid (U.Z.A.).

MNCN 37998.- Ad.- ECF.- Guadarrama.- UTM: VL/0803.- [981]m.- 09-061974.- Colectó: J.Gibter \& J.Melendro.- Donó: C. A. de Madrid (U.Z.A.).

MNCN 37999.- Ad.- ECF.- Los Molinos.- UTM: VL/0907.- [1045]m.Donó: C. A. de Madrid (U.Z.A.).

MNCN 38000.- Jv.- ECF.- Los Molinos.- UTM: VL/0907.- [1045]m.- 0612-1974.- Donó: C. A. de Madrid (U.Z.A.).

MNCN 38001.- Sad.- ECF.- Los Molinos.- UTM: VL/0907.- [1045]m.- 1807-1973.- Donó: C. A. de Madrid (U.Z.A.).

MNCN 38002.- Ad.- ECF.- Embalse de los Peñascales, Torrelodones.- UTM: VK/2392.- [740-760]m.- 16-06-1973.- Donó: C. A. de Madrid (U.Z.A.).

MNCN 38003.- Ad.- ECF.- Embalse de los Peñascales, Torrelodones.UTM: VK/2392.- [740-760]m.- 18-06-1975.- Donó: C. A. de Madrid (U.Z.A.).

MNCN 38004.- Ad.- ECF.- Torrelodones.- UTM: VK/2192.- [845]m.- 1205-1976.- Donó: C. A. de Madrid (U.Z.A.). 
MNCN 38005.- JV.- ECF.- Los Santos de la Humosa.- UTM: VK/7883,[880]m.- 19-09-1983.- Donó: C. A. de Madrid (U.Z.A.).

MNCN 38006.- Jv.- ECF.- Lozoya.- UTM: VL/3333.- [1114]m.- 10-051974.- Donó: C. A. de Madrid (U.Z.A.).

MNCN 38007.- Sad.- ECF.- Barajas, Madrid.- UTM: VK/5180.- [622]m.04-1973.- Donó: C. A. de Madrid (U.Z.A.).

MNCN 38008 - 38009.- 2Sad.- ECF.- Barajas, Madrid.- UTM: VK/5180.[622]m.- 23-10-1975.- Donó: C. A. de Madrid (U.Z.A.).

MNCN 38010.- Ad.- ECF.- Barajas, Madrid.- UTM: VK/5180.- [622]m.- 3004-1975.- Colectó: Carlos Pérez Santos.- Donó: C. A. de Madrid (U.Z.A.)

MNCN 38011.- Sad.- ECF.- Casa de Campo, Madrid.- UTM: VK/37. [600-650]m.- 07-10-1986.- Donó: C. A. de Madrid (U.Z.A.).

MNCN 38012.- Ad.- ECF.- Manzanares El Real.- UTM: VL/2708. [908]m.- 22-06-1978.- Colectó: L. Dominguez \& C. Rodriguez.- Donó: C. A. de Madrid (U.Z.A.).

MNCN 38013.- Ad.- ECF.- Montejo de la Sierra.- UTM: VL/5545.[1148]m.- 06-1969.- Donó: C. A. de Madrid (U.Z.A.).

MNCN 38015.- Sad.- ECF.- Moraleja de Enmedio.- UTM: VK/2757.[682]m.- 14-12-1980.- Donó: C.A.de Madrid (U.Z.A.).

MNCN 38016.- Ad.- ECF.- Presa del Embalse de Navacerrada, Navacerrada.- UTM: VL/1507.- [1203]m.- 04-07-1971.- Donó: C. A. de Madrid (U.Z.A.)

MNCN 38017.- Sad.- ECF.- Navalagamella.- UTM: VK/0580.- [753]m.27-06-1972.- Colectó: J.L.Ceresuela.- Donó: C. A. de Madrid (U.Z.A.)

MNCN 38018.- Ad.- ECF.- ¿Tocuelo?, Navalcarnero.- 11-04-1975.Colectó: Srta.Millá \& A. Rodriguez.- Donó: C. A. de Madrid (U.Z.A.).

MNCN 38019.- Ad.- ECF.- El Pardo, Madrid.- UTM: VK/3486.- [620720]m.- 05-06-1980.- Donó: C. A. de Madrid (U.Z.A.).

MNCN 38020.- Ad.- ECF.- Presa de pantano de San Juan, Pelayos de la Presa.- UTM: UK/8869.- [580]m.- 28-08-1975.- Donó: C. A. de Madrid (U.Z.A.).

MNCN 38021.- Sad.- ECF.- San Fernando de Henares.- UTM: VK/5475.[585]m.- 24-04-1976.- Donó: C. A. de Madrid (U.Z.A.).

MNCN 38022.- Ad.- ECF.- San Martín de la Vega.- UTM: VK/5151.[515]m.- 12-05-1973.- Donó: C. A. de Madrid (U.Z.A.).

MNCN 38023.- Sad.- ECF.- San Martín de la Vega.- UTM: VK/5151. [515]m.- 10-05-1974.- Donó: C. A. de Madrid (U.Z.A.)

MNCN 38024.- Ad.- ECF.- San Martin de la Vega.- UTM: VK/5151.[515]m.- 22-05-1975.- Donó: C. A. de Madrid (U.Z.A.).

MNCN 38025.- ECF.- Villanueva de la Cañada.- UTM: VK/1578.[652]m.- 16-04-1972.- Donó: C. A. de Madrid (U.Z.A.).

MNCN 39257 - 39258.- 2Ad.- ECF.- Montejo de la Sierra.- UTM VL/5545.- [1148]m.- 15-02-1970.- Donó: C. A. de Madrid (U.Z.A.).

MNCN 39980.- Jv.- ECF.- «El Encín», Alcalá de Henares.- UTM: VK/4744.- [610]m.- 13-01-1987.- C. A. de Madrid (U.Z.A.).

MNCN 39981.- Jv.- ECF.- Ctra. de Los Santos de la Humosa a «El Encín», Alcalá de Henares.- [610-880]m.- 28-03-1989.- Donó: C. A. de Madrid (U.Z.A.).

MNCN 41028.- Ad.- EDC, Villaviciosa de Odón.- UTM: VK/2368. [650]m.- 26-06-1982.- Colectó y donó: I. Doadrio \& P. Garzón.

MNCN 41032.- Ad.- EDC, Buitrago de Lozoya.- UTM: VL/4638.[1073]m.- 08-07-1998.- Donó: C. A. de Madrid (Centro de Recuperación de Buitrago de Lozoya)

MNCN 41033.- EDC, Buitrago de Lozoya.- UTM: VL/4638.- [1073]m.30-04-1999.- Donó: C. A. de Madrid (Centro de Recuperación de Buitrago de Lozoya).

Natrix Laurenti, 1768 (sin asignación específica)

MNCN 13197.- EDP, El Escorial.- UTM: VK/0493.- [909]m.- 1982.Colectó y donó: B. Lorenzo.

Natrix maura (Linnaeus, 1758)

MNCN 8299.- JV.- ECF.- Cerceda, El Bolao.- UTM: VL/2105.- [949]m.28-05-1980.

MNCN 8301 - 8302.- 2Ad.- ECF.- Navacerrada.- UTM: VL/1409.[1203]m.- 23-05-1980.- Colectó y donó: J. Mellado.

MNCN 8475.- Ad.- ECF.- Madrid.- UTM: VK/47.- [655]m.- -10-1912.Colectó y donó: M. Sanz de Diego.

MNCN 8477 - 8479.- Ad/Jv/Ad.- ECF.- Madrid.- UTM: VK/47.- [655]m.Colectó y donó: Sr. Cardiel.

MNCN 8492.- Sad.- ECF.- Robledo de Chavela.- UTM: UK/9584,[903]m.- 12-05-1934.- Colectó y donó: C. Bolívar.
MNCN 8496.- Jv.- ECF.- Madrid.- UTM: VK/47.- [655]m.- < [08-08-1914]. OBSERVACIONES: Pese a tener $\mathrm{n}^{0}$ de entrada 1914.08.08.03, no esta Recogido en el «Libro de registro de entradas de ejemplares en la colección de Zoología, sección de Osteozoología, 1911-1932» pp 71, por no existir anotaciones, en dicho libro, entre el 13 de julio y el 24 de septiembre, (AMNCN, 1911).

MNCN 8504.- Jv.- ECF.- Embalse de La Jarosa, Guadarrama.- UTM: VL/0502.- [1087]m.- 30-07-1976.- Colectó y donó: B. F. Bris.

MNCN 8508.- Ad.- ECF.- El Escorial.- UTM: VK/0493.- [909]m.

MNCN 8532 - 8537.- 5Ad/Jv.- ECF.- El Pardo, Madrid.- UTM: VK/3486.[620-720]m.- 23/25-04-1907.- Colectó y donó: J. Árias Encobet.

MNCN 8561 - 8566.- 2JvSad/3Ad.- ECF.- El Pardo, Madrid.- UTM: VK/3486.- [620-720]m.- 04-1907.- Colectó y donó: J. Árias Encobet.

MNCN 8578.- JV.- ECF.- Peñalara (*), Rascafría.- VL/1922.- Colectó y Donó: [M.L. Esteban \& J. Dorda].

ObSERVACIONES: (*) Se encontraba en un frasco en el que había una etiqueta que decía: Lacerta viridis (Peñalara), no había lagarto, pero sí otro joven de Coronella girondica (MNCN 9137). En cualquier caso todo ello debe tratarse de un error de etiquetación, ya que ni Coronella girondica ni Natrix maura, se distribuyen en Peñalara.

MNCN 8621 - 8624.- 4Jv.- ECF.- Madrid.- UTM: VK/47.- [655]m.- 1880.Colectó y donó: Sr. Cardiel.

MNCN 8634.- Sad.- ECF.- El Pardo, Madrid.- UTM: VK/3486.- [620720]m.- <[21-03-1912].- Colectó y donó: F. Árias. OBSERVACIONES: Pese a tener $\mathrm{n}^{\circ}$ de entrada 1912.03.21, no esta Recogidos en el «Libro de registro de entradas de ejemplares en la colección de Zoología, sección de Osteozoología, 1911-1932» pp 41, (AMNCN, 1911).

MNCN 9108.- Ad.- ECF.- Villalba, Collado-Villalba.- UTM: VK/1699.[917]m.- -1880.- Colectó y donó: E. Boscá.

OBSERVACIONES: Recogido en el "Libro de registro de entradas de ejemplares en la colección de Zoología, sección de Osteozoología, 19111932" pp 65. como "Tropidonotus viperinus", (AMNCN, 1911).

MNCN 9116.- Sad.- ECF.- Río Manzanares, Madrid.- UTM: VK/37.[590]m.- 10-07-1978.- Colectó y donó: I. Doadrío.

MNCN 9121.- Jv.- ECF.- Aranjuez.- UTM: VK/4832.- [494]m.- 09-091975.- Colectó y donó: I. Doadrío.

MNCN 9169.- Ad.- ECF.- Madrid.- UTM: VK/47.- [655]m.- 18-12-1984.

MNCN 9239.- JV.- ECF.- Aranjuez.- UTM: VK/4832.- [494]m.- 30-091975.- Colectó y donó: I. Doadrío.

MNCN 15863.- Jv.- ECF.- Embalse de El Pontón de la Oliva, Patones.UTM: VL/6226.- [726]m.- 10-09-1983.- Colectó y donó: J. C. Hidalgo.

MNCN 15879.- Ad.- ECF.- ¿Finca La Grajilla?, El Escorial.- 21-07-1982.Colectó y donó: A. Mújica Nava.

MNCN 16374.- Ad.- EDC.- Laguna de Ontígola, Aranjuez.- UTM: VK/4930.- [560]m.- 13-05-1983.- Colectó y donó: R. R. Talavera \& J. C. Arrechea.

MNCN 16376.- Ad.- PCF - EDP.- Embalse de Valmayor, Galapagar.UTM: VK/18.- [831]m.- 03-06-1984.- Colectó y donó: L. M. Guio.

MNCN 16377.- Ad.- PCF - EDP, San Agustín de Guadalix.- UTM: VL/4703.- [684]m.- 27-06-1984.- Colectó y donó: R. R. Talavera \& J. C. Arrechea.

MNCN 16378.- Ad.- PCF - EDP, San Agustín de Guadalix.- UTM: VL/4703.- [684]m.- 15-07-1984.- Colectó y donó: R. R. Talavera \& J. C. Arrechea.

MNCN 16379.- Ad.- PCF - EDP.- Laguna de Ontígola, Aranjuez.- UTM: VK/4930.- [560]m.- 13-05-1983.- Colectó y donó: R. R. Talavera \& J. C. Arrechea.

MNCN 16380.- Ad.- PCF - EDP.- Laguna de Ontígola, Aranjuez.- UTM: VK/4930.- [560]m.- 13-05-1983.- Colectó y donó: R. R. Talavera \& J. C. Arrechea.

MNCN 16383.- Ad.- PCF - EDP, Paredes de Buitrago.- UTM: VL/5239.[1020]m.- 04-07-1983.- Colectó y donó: R. R. Talavera \& A. Corcuera.

MNCN 16385.- Ad. PCF - EDP, San Agustín de Guadalix - UTM: VL/4703.- [684]m.- 10-06-1984.- Colectó y donó: R. R. Talavera \& J. C. Arrechea.

MNCN 16395.- JV.- EDP, Hoyo de Manzanares.- UTM: VK/2397.[1001]m.- 16-09-1983.- Colectó y donó: R. R. Talavera \& B. Sanchiz.

MNCN 16396.- Ad.- EDP, Hoyo de Manzanares.- UTM: VK/2397.[1001]m.- 16-09-1983.- Colectó y donó: R. R. Talavera \& B. Sanchiz.

MNCN 16398.- JV.- PCF - EDP, San Agustín de Guadalix.- UTM: VL/4703.[684]m.- 23-04-1983.- Colectó y donó: R. R. Talavera \& J. C. Arrechea.

MNCN 16399.- JV.- PCF - EDP, San Agustín de Guadalix.- UTM: VL/4703.[684]m.- 30-04-1983.- Colectó y donó: R. R. Talavera \& J. C. Arrechea. 
MNCN 16400.- Jv.- PCF - EDP, San Agustín de Guadalix.- UTM: VL/4703.[684]m.- 30-04-1983.- Colectó y donó: R. R. Talavera \& J. C. Arrechea.

MNCN 16403.- Ad.- PCF - EDP, El Berrueco.- UTM: VL/5326.- [925]m.-06-1984.- Colectó y donó: B. Arano.

MNCN 16404.- JV.- PCF - EDP.- Embalse de los Peñascales, Torrelodones.- UTM: VK/2392.- [740-760]m.- 11-3-1983.- Colectó y donó: R. R. Talavera \& A. Corcuera.

MNCN 16405.- Ad.- PCF - EDP, San Agustín de Guadalix.- UTM: VL/4703.- [684]m.- 23-04-1983.- Colectó y donó: R. R. Talavera \& J. C Arrechea.

MNCN 16406.- JV.- PCF - EDP, San Agustín de Guadalix.- UTM VL/4703.- [684]m.- 30-04-1983.- Colectó y donó: R. R. Talavera \& J. C. Arrechea.

MNCN 16407.- Ad.- PCF - EDP, San Agustín de Guadalix.- UTM VL/4703.- [684]m.- 30-04-1983.- Colectó y donó: R. R. Talavera \& J. C. Arrechea.

MNCN 16408.- Jv.- PCF - EDP, San Agustín de Guadalix.- UTM: VL/4703.- [684]m.- < [09-11-1984].- Colectó y donó: R. R. Talavera \& J. C. Arrechea.

MNCN 16411.- Ad.- EDC, Madrid.- UTM: VK/47.- [655]m.- 06-1983.Donó: Grupo Herpetológico Zona Centro (G.H.E.Z.O.C).

MNCN 17915 - 17923.- 9(Ad+Sad+Jv).- ECF.- San Martín de Valdeiglesias.- UTM: UK/8169.- [681]m.- verano-1979.- Colectó y donó: C. E. Pérez Santos.

MNCN 23907.- Ad.- PCF - EDP.- Sierra de Guadarrama.- 1983.

MNCN 38088.- Sad.- ECF.- Collado-Villalba.- UTM: VK/1699.- [917]m.20-07-1974.- Donó: C. A. de Madrid (U.Z.A.).

MNCN 38368.- Ad.- ECF.- Embalse de El Atazar.- UTM: VL/52.- [870]m.27-05-1975.- Donó: C. A. de Madrid (U.Z.A.)

MNCN 38369.- Ad.- ECF.- Embalse del Río Sequillo, Buitrago de LozoyaGargantilla de L.- UTM: VL/43.- [1010]m.- 27-05-1976.- Donó: C. A de Madrid (U.Z.A.).

MNCN 38370.- Ad.- ECF.- Río Jarama.- 21-06-1970.- Donó: C. A. de Madrid (U.Z.A.).

MNCN 38371.- Jv.- ECF.- Río Henares, Alcalá de Henares.- UTM VK/67.- [590]m.- -05-1980.- C. A. de Madrid (U.Z.A.)

MNCN 38372.- Jv.- ECF.- Alcalá de Henares.- UTM: VK/6881.- [587]m.05-02-1981.- Donó: C. A. de Madrid (U.Z.A.).

MNCN 38373.- Jv.- ECF.- Alpedrete.- UTM: VL/1301.- [919]m.- 11-051972.- Colectó: F. Parra.- C. A. de Madrid (U.Z.A.).

MNCN 38374.- Jv.- ECF.- Aranjuez.- UTM: VK/4832.- [494]m.- 30-091975.- Donó: C. A. de Madrid (U.Z.A.).

MNCN 38375 - 38376.- 2Ad.- ECF.- Laguna de Ontígola, Aranjuez. UTM: VK/4930.- [560]m.- 12-03-1977.- C. A. de Madrid (U.Z.A.).

MNCN 38377 - 38378.- Ad/Sad.- ECF.- Canencia.- UTM: VL/3729.[1150]m.- 02/10-07-1970.- Donó: C. A. de Madrid (U.Z.A.)

MNCN 38379.- Ad.- ECF.- Cercedilla.- UTM: VL/1010.- [1188]m.- 18-071972.- Donó: C. A. de Madrid (U.Z.A.).

MNCN 38380.- Ad.- ECF.- Collado-Villalba.- UTM: VK/1699.- [917]m.16-05-1974.- Donó: C. A. de Madrid (U.Z.A.).

MNCN 38381 - 38384.- 2Ad/Sad/Ad.- ECF.- El Escorial.- UTM VK/0493.- [909]m.- 08-1970.- Donó: C. A. de Madrid (U.Z.A.).

MNCN 38385.- Ad.- ECF.- Río Guadarrama a $10 \mathrm{~km}$ de, El Escorial. UTM: 10-08-1970.- Donó: C. A. de Madrid (U.Z.A.).

MNCN 38386.- Ad.- ECF.- Ciudad Deportiva La Dehesa (Cuatrovientos), Madrid.- UTM: VK/3270.- [670]m.- 13-10-1977.- Donó: C. A. de Madrid (U.Z.A.)

MNCN 38387.- Jv.- ECF.- Los Molinos.- UTM: VL/0907.- [1045]m.- 1607-1973.- Donó: C. A. de Madrid (U.Z.A.).

MNCN 38388.- Ad.- ECF.- Lozoyuela.- UTM: VL/4831.- [1026]m.- 2206-1975.- Donó: C. A. de Madrid (U.Z.A.).

MNCN 38389.- Ad.- ECF.- Cuatrovientos, Madrid.- UTM: VK/3270.[690]m.- 19-05-1974.- Donó: C. A. de Madrid (U.Z.A.)

MNCN 38390.- Ad.- ECF.- Río Jarama, Madrid.- UTM: VK/57-59.[540]m.- 07-08-1980.- Donó: C. A. de Madrid (U.Z.A.)

MNCN 38391.- JV.- ECF.- Río Manzanares, Madrid.- UTM: VK/37. [590]m.- 11-05-1972.- Colectó: Rafael Escribano.- Donó: C. A. de Madrid (U.Z.A.)

MNCN 38392.- Ad.- ECF.- Embalse de Santillana, Manzanares El Real.UTM: VL/2808.- [900]m.- 28-07-1970.- Donó: C. A. de Madrid (U.Z.A.).

MNCN 38393.- Ad.- ECF.- Móstoles.- UTM: VK/2664.- [661]m.- 27-091970.- Colectó: J.López.- Donó: C. A. de Madrid (U.Z.A.)
MNCN 38394.- Ad.- ECF.- Rascafría.- UTM: VL/2528.- [1163]m.- 30-051974 - Donó: C. A. de Madrid (U.Z.A.)

MNCN 38395.- Sad.- ECF.- Rascafría.- UTM: VL/2528.- [1163]m.- 16-041979.- Donó: C. A. de Madrid (U.Z.A.).

MNCN 38396.- Ad.- ECF.- Rascafría.- UTM: VL/2528.- [1163]m.Prim/Ver-1979.- Donó: C. A. de Madrid (U.Z.A.).

MNCN 38397.- Ad.- ECF.- Río Lozoya cruce con el puente de Taboada, Gargantilla del Lozoya.- UTM: VL/3032.- [1000]m.- 23-05-1976.Donó: C. A. de Madrid (U.Z.A.)

MNCN 38398.- Jv.- ECF.- Río Lozoya, Rascafría.- 21-08-1979.- Colectó: J. Lobón Cerviá.- Donó: C. A. de Madrid (U.Z.A.).

MNCN 38399.- Ad.- ECF.- Robledo de Chavela.- UTM: UK/9584.[903]m.- 01-08-1975.- Donó: C. A. de Madrid (U.Z.A.).

MNCN 38400.- Ad.- ECF.- San Martín de la Vega.- UTM: VK/5151.[515]m.- 26-05-1974.- Donó: C. A. de Madrid (U.Z.A.).

MNCN 38401.- Ad.- ECF.- Finca de los Ángeles, San Martín de la Vega.UTM: VK/5457.- [540]m.- 27-05-1973.- Colectó: M. Cuesta Pons.Donó: C. A. de Madrid (U.Z.A.).

MNCN 38402 - 38403.- 2Ad.- ECF.- Finca de los Ángeles, San Martín de la Vega.- UTM: VK/5457.- [540]m.- 26/30-06-1973.- Donó: C. A. de Madrid (U.Z.A.).

MNCN 38404.- Ad.- ECF.- Santa María de la Alameda.- UTM: UK/9394.[1460]m.- 21-09-1976.- C. A. de Madrid (U.Z.A.).

MNCN 38405.- Ad.- ECF.- Embalse de los Peñascales, Torrelodones.- UTM: VK/2392.- [740-760]m.- 27-07-1972.- Donó: C. A. de Madrid (U.Z.A.).

MNCN 38406.- JV.- ECF.- Embalse de los Peñascales, Torrelodones.- UTM: VK/2392.- [740-760]m.- 13-05-1973.- Donó: C. A. de Madrid (U.Z.A.).

MNCN 38407 - 38412.- 5(Av+Sad).- ECF.- Embalse de los Peñascales, Torrelodones.- UTM: VK/2392.- [740-760]m.- 20-05-1973.- Colectó: J.Ibañez \& L.Rivas..- Donó: C. A. de Madrid (U.Z.A.)

MNCN 38413 - 38416.- 4Ad.- ECF.- Embalse de los Peñascales, Torrelodones.- UTM: VK/2392.- [740-760]m.- 16-06-1973.- Colectó: J. bañez \& L. Rivas.- Donó: C. A. de Madrid (U.Z.A.).

MNCN 38417.- Ad.- ECF.- Embalse de los Peñascales, Torrelodones.UTM: VK/2392.- [740-760]m.- 17-06-1973.- Colectó: L. Rivas.- Donó: C. A. de Madrid (U.Z.A.).

MNCN 38418 - 38419.- Ad/Jv.- ECF.- Embalse de los Peñascales, Torrelodones.- UTM: VK/2392.- [740-760]m.- 24-06-1973.- Donó: C. A. de Madrid (U.Z.A.).

MNCN 38420.- Ad.- ECF.- Embalse de los Peñascales, Torrelodones.UTM: VK/2392.- [740-760]m.- 01-07-1973.- Colectó: L. Rivas.- Donó: C. A. de Madrid (U.Z.A.).

MNCN 38423 - 38434.- 12(Ad+Jv).- ECF.- Embalse de los Peñascales, Torrelodones.- UTM: VK/2392.- [740-760]m.- 11-05-1974.- Donó: C. A. de Madrid (U.Z.A.).

MNCN 38435 - 38437- 3Ad- ECF- Embalse de los Peñascales, Torrelodones.- UTM: VK/2392.- [740-760]m.- 25-05-1974.- Donó: C. A. de Madrid (U.Z.A.).

MNCN 38438.- JV.- ECF.- Embalse de los Peñascales, Torrelodones.UTM: VK/2392.- [740-760]m.- 28-09-1974.- Donó: C. A. de Madrid (U.Z.A.).

MNCN 38439 - 38440.- Ad/Jv.- ECF.- Embalse de los Peñascales, Torrelodones.- UTM: VK/2392.- [740-760]m.- 18-06-1975.- Donó: C. A. de Madrid (U.Z.A.).

MNCN 38441.- Ad.- ECF.- Embalse de los Peñascales, Torrelodones.- UTM: VK/2392.- [740-760]m.- 15-04-1976.- Donó: C. A. de Madrid (U.Z.A.).

MNCN 38442 - 38444.- Jv/2Ad. ECF.- Embalse de los Peñascales, Torrelodones.- UTM: VK/2392.- [740-760]m.- 06-09-1976.- Donó: C. A. de Madrid (U.Z.A.)

MNCN 38445.- Jv.- ECF.- Valdemorillo.- UTM: VK/0894.- [815]m.- 2804-1975.- Colectó: Sr. Baragaño.- Donó: C. A. de Madrid (U.Z.A.).

MNCN 39273 - 39276.- 4Ad.- ECF.- Embalse de los Peñascales, Torrelodones.- UTM: VK/2392.- [740-760]m.- 08-09-1973.- Donó: C. A. de Madrid (U.Z.A.)

MNCN 39277.- Sad.- ECF.- ¿Urbanización Nueva Sierra?, Campo Real.UTM: 19-04-1973.- Donó: C. A. de Madrid (U.Z.A.).

MNCN 40020.- Ad.- ECF.- Ctra. De Los Santos de la Humosa a «El Encín», Alcalá de Henares.- [610-880]m.- 16-11-1984.- Donó: C. A. de Madrid (U.Z.A.).

MNCN 40028.- Sad.- ECF.- Río Lozoya-- 18-05-1943.- Donó: C. A. de Madrid (U.Z.A.).

MNCN 40038.- Jv.- ECF.- Río Manzanares, El Pardo, Madrid.- UTM: VK/38.- [620]m.- 20-08-1941.- Donó: C. A. de Madrid (U.Z.A.). 
MNCN 40042 - 40043.- 2Jv.- ECF.- Río Jarama, San Fernando de Henares.UTM: VK/57.- [580]m.- 08/12-08-1943.- Donó: C. A. de Madrid (U.Z.A.)

MNCN 41505.- Ad.- ECF.- Presa del Romeral, San Lorenzo de El Escorial.UTM: VK/0294.- [1100]m.- 06-05-1989.- Colectó: D. Moreno.

MNCN 41538.- JV.- ECF.- Pelayos de la Presa.- UTM: UK/8568.- [570]m.$<$ [12-11-2002].- Colectó: C. Olmos.

Natrix natrix (Linnaeus, 1758)

MNCN 8661.- JV-.- ECF.- Madrid.- UTM: VK/47.- [655]m.- Colectó y donó: I. Bolívar.

MNCN 9049.- Ad - ECF.- Madrid - UTM: VK/47 - [655]m.

MNCN 9128.- Ad.- ECF.- Río Lozoya cruce con el puente de Taboada, Gargantilla del Lozoya.- UTM: VL/3032.- [1000]m.- 23-05-1976. Colectó y donó: I. Doadrío.

MNCN 9129.- Ad.- ECF.- Río Lozoya cruce con el puente de Taboada, Gargantilla del Lozoya.- UTM: VL/3032.- [1000]m.- 27-05-1976.Colectó y donó: I. Doadrío.

MNCN 16418.- Ad.- PCF.- Patones de Abajo, Patones.- UTM: VL/5923.[710]m.- 30-07-1984.- Colectó: A. Panadero.

MNCN 18730.- Ad.- ECF.- Sierra de Guadarrama, La Cabrera.- UTM VL/4824.- 1050m.- 17-11-1990.- Colectó y donó: A. Compte Sart.

MNCN 20093.- Ad.- ECF.- El Ventorrillo, Mancomunidad de Cercedilla y Navacerrada.- UTM: VL/1312.- [1500]m.- 03-07-1980.- Colectó y donó: J. Mellado.

MNCN 24373.- Ad.- ECF.- El Ventorrillo, Mancomunidad de Cercedilla y Navacerrada.- UTM: VL/1312.- [1500]m.- 01-09-1986.

MNCN 38716.- Sad.- ECF.- Collado-Villalba.- UTM: VK/1699.- [917]m.13-07-1980.- Donó: C. A. de Madrid (U.Z.A.).

MNCN 38717.- Ad.- ECF.- Guadarrama.- UTM: VL/0803.- [981]m.- Sin fecha.- Donó: C. A. de Madrid (U.Z.A.).

MNCN 38718.- Ad.- ECF.- Lozoya.- UTM: VL/3333.- [1114]m.- 25-091979.- Donó: C. A. de Madrid (U.Z.A.).

MNCN 38719.- Ad.- ECF.- Sur de la Maliciosa. Sierra de Guadarrama., Mataelpino.- UTM: VL/11.- [1200-2200]m.- 24-08-1975.- Donó: C. A. de Madrid (U.Z.A.).

MNCN 38720.- Sad.- ECF.- Rascafría.- UTM: VL/2528.- [1163]m.- 16-041979.- Donó: C. A. de Madrid (U.Z.A.).

MNCN 38721.- Ad.- ECF.- Pantano de San Juan, San Martín de Valdeiglesias.- UTM: UK/87.- [580]m.- Verano-1975.- Donó: C. A. de Madrid (U.Z.A.)

MNCN 38722.- Ad.- ECF.- Villanueva de la Cañada.- UTM: VK/1578. [652]m.- 24-08-1972.- Colectó: J.L.Ceresuela.- Donó: C. A. de Madrid (U.Z.A.).

MNCN 41510.- Ad.- ECF.- Pelayos de la Presa.- UTM: UK/8568.[570]m.- <[20-10-2000].- Colectó: C. Olmos.

Familia Viperidae Oppel, 1811

Vipera latasti Boscá, 1878

MNCN 6423.- Sad.- ECF.- El Ventorrillo, Mancomunidad de Cercedilla y Navacerrada.- UTM: VL/1312.- [1500]m.- 04-07-1980.- Colectó y donó: J. Mellado.

MNCN 8974.- Sad.- ECF.- Cercedilla.- UTM: VL/1010.- [1188]m.- -081910.- Colectó: C. Bolívar.

MNCN 8979.- Sad.- ECF.- Navacerrada.- UTM: VL/1409.- [1203]m.- -041910.- Colectó: T. Rico Jimeno.- Donó: T. Rico Jimeno.

MNCN 9182.- Ad.- ECF.- Cerro Colgado, Cercedilla.- UTM: VL/1011.[1347]m.- 20-06-1976.
MNCN 16429.- Ad.- PCF - EDP.- El Ventorrillo, Mancomunidad de Cercedilla y Navacerrada.- UTM: VL/1312.- [1500]m.- 31-06-1983.Colectó y donó: A. Larramendi.

MNCN 19108.- Ad.- ECF.- El Ventorrillo, Mancomunidad de Cercedilla y Navacerrada.- UTM: VL/1312.- [1500]m.- 24-03-1994.- Colectó y donó: A. Salvador.

MNCN 19405.- Sa.- ECF.- Puerto de Canencia, Bustarviejo-Canencia.UTM: VL/3524.- [1600]m.- 26-05-1986.- Colectó y donó: A. Mújica Nava.

MNCN 39037.- Sad.- ECF.- Horcajuelo de la Sierra.- UTM: VL/5445.[1145]m.- 11-10-1971.- Donó: C. A. de Madrid (U.Z.A.).

MNCN 39038.- Ad.- ECF.- Manzanares El Real.- UTM: VL/2708.[908]m.- 23-10-1971.- Donó: C. A. de Madrid (U.Z.A.).

MNCN 39039.- Sad.- ECF.- La Pedriza, Manzanares El Real.- UTM: VL/21 - [1400-1700]m - 26-05-1974 - Donó: C. A de Madrid (U.Z.A.)

MNCN 39040.- Ad.- ECF.- La Maliciosa, Sierra del Guadarrama, Mataelpino.- UTM: VL/1813.- [2200]m.- 26-04-1970.- Donó: C. A. de Madrid (U.Z.A.).

MNCN 39041.- Ad.- ECF.- El Chaparral, Montejo de la Sierra.- UTM: VL/5850.- [1470]m.- 06-06-1968.- Colectó: J. Garzón.- Donó: C. A. de Madrid (U.Z.A.).

MNCN 39042.- ECF.- Montejo de la Sierra.- UTM: VL/5545.- [1148]m.31-03-1969.- Donó: C. A. de Madrid (U.Z.A.).

MNCN 39043.- ECF.- Montejo de la Sierra.- UTM: VL/5545.- [1148]m.05-1969.- Donó: C. A. de Madrid (U.Z.A.).

MNCN 39044.- Ad.- ECF.- Montejo de la Sierra.- UTM: VL/5545.[1148]m.- -08-1969.- Donó: C. A. de Madrid (U.Z.A.).

MNCN 39045.- Sa.- ECF.- El Chaparral, Montejo de la Sierra.- UTM: VL/5850.- [1470]m.- 28-05-1970.- Colectó: F. Palacios.- Donó: C. A. de Madrid (U.Z.A.).

MNCN 39046.- Ad.- ECF.- Montejo de la Sierra.- UTM: VL/5545.[1148]m.- 27-03-1971.- Donó: C. A. de Madrid (U.Z.A.).

MNCN 39047.- Ad.- ECF.- Montejo de la Sierra.- UTM: VL/5545.[1148]m.- 01/10-10-1971.- Donó: C. A. de Madrid (U.Z.A.).

MNCN 39048.- ECF.- Montejo de la Sierra.- UTM: VL/5545.- [1148]m.Verano-1972.- Donó: C. A. de Madrid (U.Z.A.)

MNCN 39049.- Sad.- ECF.- Montejo de la Sierra.- UTM: VL/5545.[1148]m.- 20-07-1972.- Donó: C. A. de Madrid (U.Z.A.).

MNCN 39050.- Sa.- ECF.- Montejo de la Sierra.- UTM: VL/5545.[1148]m.- 07-10-1975.- Donó: C. A. de Madrid (U.Z.A.).

MNCN 39051.- Sa.- ECF.- Montejo de la Sierra.- UTM: VL/5545.[1148]m.- 25-05-1973.- Donó: C. A. de Madrid (U.Z.A.).

MNCN 39052 - 39053.- -/Sa.- ECF.- Montejo de la Sierra.- UTM: VL/5545.- [1148]m.- 18-07-1978.- Donó: C. A. de Madrid (U.Z.A.).

MNCN 39054.- Ad.- ECF.- Montejo de la Sierra.- UTM: VL/5545.[1148]m.- 28-05-1970.- Donó: C. A. de Madrid (U.Z.A.).

MNCN 39055.- Ad.- ECF.- Montejo de la Sierra.- UTM: VL/5545.[1148]m.- 03-04-1980.- Donó: C. A. de Madrid (U.Z.A.).

MNCN 39056.- Sad.- ECF.- Peguerinos.- UTM: UK/9598.- [1350]m.- 0810-1978.- Donó: C. A. de Madrid (U.Z.A.).

MNCN 39057.- Ad.- ECF.- En el Km 6 de la M-130 (=carretera de la Puebla de la Sierra a Pradena del Rincón), Pradena del Rincón.- UTM: VL/5944 - [1420]m.- 03-08-1973.- Donó: C. A. de Madrid (U.Z.A.)

MNCN 39073.- Ad.- ECF.- La Peñota, Villavieja de Lozoya.- UTM: VL/3644.- [1919]m.- 13-10-1973.- Donó: C. A. de Madrid (U.Z.A.) 